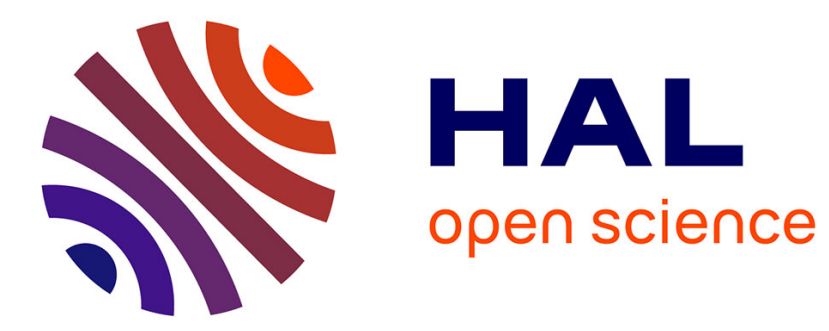

\title{
Estimation des dimensions de certaines variétés de Kisin Xavier Caruso
}

\section{To cite this version:}

Xavier Caruso. Estimation des dimensions de certaines variétés de Kisin. Journal für die reine und angewandte Mathematik, 2017, 723 (723), pp.1-77. 10.1515/crelle-2014-0066 . hal-00483259v2

\section{HAL Id: hal-00483259 \\ https://hal.science/hal-00483259v2}

Submitted on 30 Jan 2011

HAL is a multi-disciplinary open access archive for the deposit and dissemination of scientific research documents, whether they are published or not. The documents may come from teaching and research institutions in France or abroad, or from public or private research centers.
L'archive ouverte pluridisciplinaire HAL, est destinée au dépôt et à la diffusion de documents scientifiques de niveau recherche, publiés ou non, émanant des établissements d'enseignement et de recherche français ou étrangers, des laboratoires publics ou privés. 


\title{
Estimation des dimensions de certaines variétés de Kisin
}

\author{
Xavier Caruso
}

Mai 2010

\begin{abstract}
Résumé
Dans cet article, nous nous intéressons aux dimensions de certaines variétés qui ont été introduites récemment par Kisin pour démontrer la modularité de certaines représentations galoisiennes. Nous étudions plus spécialement un cas particulier pour lequel nous donnons une estimation de la dimension en question, puis, en nous basant sur ce résultat, nous énonçons une conjecture dans le cas général.
\end{abstract}

\section{Abstract}

In this paper, we study dimensions of some varieties, that were introduced recently by Kisin in order to prove modularity of some Galois representations. In fact, we mainly consider a special case for which we obtain an estimation of the dimension we are interested in. Then, based on this result, we state a conjecture for the general case.

\section{Table des matières}

1 Une stratification utile

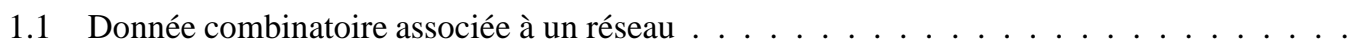

1.2 Paramétrisation de l'espace des fonctions $\varphi \ldots \ldots \ldots \ldots \ldots \ldots \ldots \ldots$

1.3 Les variétés $\mathcal{X}_{\varphi}$ et leurs dimensions $\ldots \ldots \ldots \ldots \ldots \ldots \ldots$

2 Mise en place de la méthode 23

2.1 Préliminaires de programmation linéaire . . . . . . . . . . . . . . . . . . . . . . . . . .

2.2 Étude du cône convexe $\Phi \ldots \ldots \ldots \ldots \ldots \ldots \ldots \ldots$

2.3 Un premier exemple d'application .............................. 29

3 Dimension des variétés $\mathcal{X}_{\leqslant e}, \mathcal{X}_{\mu}$ et $\mathcal{X}_{\leqslant \mu}$

3.1 Les points extrémaux de $A_{Q_{\max }, g, \ell} \ldots \ldots \ldots \ldots \ldots \ldots \ldots \ldots$

3.2 Le calcul de $A_{Q, g, \ell} \ldots \ldots \ldots \ldots \ldots \ldots \ldots \ldots$

3.3 Démonstration des théorèmes

3.4 Points extrémaux de $A_{Q, g, \ell} \cap C$ : quelques exemples ................. 44

4 Perspectives et conjectures 47

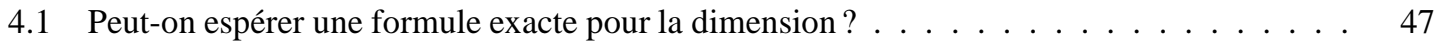

4.2 Généralisations envisageables ........................... 51

Motivé par l'étude de certains problèmes de modularité et poursuivant des travaux de Breuil, Kisin a introduit et étudié dans [7] une certain nombre de variétés, notées $\mathscr{G}_{V_{\mathbb{F}}}, \mathscr{G} \mathscr{R}_{V_{\mathbb{F}}, 0}$ et $\mathscr{G} \mathscr{R}_{V_{\mathbb{F}}, 0}^{\mathbf{v}, \text { loc }}$ dans loc. cit., paramétrant certains types de schémas en groupes définis sur l'anneau des entiers d'un corps local $K$ d'inégale caractéristique $(0, p)$. Dans ce qui précède, l'indice $V_{\mathbb{F}}$ désigne une représentation du groupe de Galois absolu de $K$ à coefficients dans un corps fini $\mathbb{F}$ de caractéristique $p$. S'inspirant de cette construction, Pappas et Rapoport ont ensuite défini dans [15] un champ sur $\mathbb{Z}_{p}$ dont certaines fibres s'interprètent comme les variétés que Kisin avait définies, et en ont profité pour nommer ces dernières variétés de Kisin. Comprendre la géométrie des variétés de Kisin, et notamment calculer leurs dimensions, est d'une grande 
importance pour les applications. Toutefois, en dehors du cas où $V_{\mathbb{F}}$ est de dimension 2 considéré dans certains travaux de Kisin (voir [7]), Hellmann ([5], [6]) et Imai ([9], [10], [11]), pratiquement rien n'est connu. Dans cet article, nous entâmons l'étude de la dimension des variétés de Kisin lorsque la représentation galoisienne $V_{\mathbb{F}}$ est de dimension supérieure à 2. Plus précisément, dans un premier temps, nous donnons des estimations de ces dimensions dans l'exemple (déjà compliqué) où le corps des coefficients $\mathbb{F}$ est le corps premier $\mathbb{F}_{p}$ et où le groupe de Galois agit trivialement sur $V_{\mathbb{F}}$ et, forts de cela, dans un second temps, nous formulons un certain nombre de conjectures générales qui laissent croire que le cas particulier étudié est plutôt représentatif.

Afin de décrire plus en détails le contenu de cet article, il est nécessaire de commencer par rappeler la définition des variétés de Kisin. Soit $p$ un nombre premier et $k$ un corps de caractéristique $p$. On pose $K=k((u))$ et on appelle $\phi$ l'unique morphisme de $k$-algèbres $\phi: K \rightarrow K$ qui est continu pour la topologie $u$-adique et qui envoie $u$ sur $u^{p}$. Pour tout l'article, on fixe un nombre entier $d$ supérieur ou égal à 1 et on pose $M=K^{d}$. Le Frobenius $\phi$ s'étend naturellement en un opérateur sur $M$, encore noté $\phi$, en agissant coordonnée par coordonnée 1 . Un réseau $L$ de $M$ est, par définition, un sous- $k[[u]]$-module $L \subset M$ engendré par une $k((u))$-base de $M$. On note $\mathcal{L}_{\leqslant e}$ l'ensemble des réseaux $L$ de $M$ vérifiant la condition

$$
u^{e} L \subset \phi\left(k\left[\left[u^{1 / p}\right]\right] \otimes_{k[[u]]} L\right) \subset L
$$

où $\phi$ est étendu à $k\left[\left[u^{1 / p}\right]\right] \otimes_{k[[u]]} L$ de façon évidente. Kisin démontre que $\mathcal{L}_{\leqslant e}$ apparaît naturellement comme les $k$-points d'une variété algébrique notée $\mathcal{X}_{\leqslant e}$. Dans cet article, nous démontrons le théorème suivant.

Théorème 1. Avec les notations précédentes, on $a$ :

$$
\left[\frac{d^{2}}{4}\right] \cdot\left[\frac{e-p+2}{p+1}\right] \leqslant \operatorname{dim}_{k} \mathcal{X}_{\leqslant e} \leqslant \frac{d(d-1)}{2}+\left[\frac{d^{2}}{4}\right] \cdot \frac{e}{p+1}
$$

où $[x]$ désigne la partie entière du réel $x$.

On insiste sur le fait que le théorème n'affirme en aucune façon que les variétés $\operatorname{dim}_{k} \mathcal{X}_{\leqslant e}$ sont équidimensionnelles, ni même que l'inégalité annoncée vaut pour toutes les composantes irréductibles. Le nombre $\operatorname{dim}_{k} \mathcal{X}_{\leqslant e}$ désigne bien uniquement la plus grande dimension d'une composante irréductible.

En réalité, à la place du théorème 11, nous allons démontrer un résultat légèrement plus général que nous énonçons maintenant. On se donne deux entiers $h$ et $b$ avec $b \geqslant 2$, et on remplace $\phi$ par l'application $\sigma: k((u)) \rightarrow k((u))$ donnée par la formule suivante :

$$
\sigma: K \rightarrow K, \quad \sum_{i \gg-\infty} a_{i} u^{i} \mapsto \sum_{i \gg-\infty} a_{i}^{p^{h}} u^{b i} .
$$

Lorsque $h=0$ et $b=p$, on retrouve l'opérateur $\phi$. Un autre cas qui semble intéressant est celui où $h=1$ et $b=p$. En effet, un théorème de Breuil (voir [1]) dit alors que les éléments de $\mathcal{X}_{\leqslant e}(k)$ sont en bijection avec l'ensemble des classes d'isomorphisme de modèles entiers du schéma en groupes $(\mathbb{Z} / p \mathbb{Z})_{K}^{d}$ où $K$ est une extension totalement ramifiée fixée de degré $e$ de Frac $W(k)$ (où $W(k)$ désigne l'anneau des vecteurs de Witt à coefficients dans $k$ ). Le cas $b=1$ est, à vrai dire, lui aussi très intéressant. Nous avons préféré l'écarter dans cet article simplement car il conduit à certaines variétés de Deligne-Lusztig affines qui ont déjà été largement étudiées et, en particulier, dont les dimensions ont déjà été déterminées (dans une plus grande généralité) dans les articles [4] et [16].

Théorème 2. Si $h \neq 0$, on $a$ :

$$
\left[\frac{d^{2}}{4}\right] \cdot\left[\frac{e-b+2}{b+1}\right] \leqslant \operatorname{dim}_{k} \mathcal{X}_{\leqslant e} \leqslant\left[\frac{d^{2}}{4}\right] \cdot \frac{e}{b+1} .
$$

Si $h=0$, on $a$ :

$$
\left[\frac{d^{2}}{4}\right] \cdot\left[\frac{e-b+2}{b+1}\right] \leqslant \operatorname{dim}_{k} \mathcal{X}_{\leqslant e} \leqslant \frac{d(d-1)}{2}+\left[\frac{d^{2}}{4}\right] \cdot \frac{e}{b+1} .
$$

1. C'est le choix de cette action particulière de $\phi$ sur $M$ qui correspond au fait que l'on se restreint à l'action triviale de Galois sur l'espace $V_{\mathbb{F}}$. 
Dans leurs articles respectifs, Kisin d'une part et Pappas et Rapoport d'autre part définissent également des variantes des variétés $\mathcal{X}_{\leqslant e}$ qui ne sont plus paramétrées par un unique entier $e$ mais par un $d$-uplet d'entiers relatifs $\left(\mu_{1}, \ldots, \mu_{d}\right)$ tels que $\mu_{1} \geqslant \cdots \geqslant \mu_{d}$. Dans la généralité considérée ici — c'est-à-dire lorsque $b$ et $h$ peuvent être quelconques - ces variantes ont encore un sens. Précisément, si $\mu=\left(\mu_{1}, \ldots, \mu_{d}\right)$ est un $d$-uplet comme précédemment, on peut construire des variétés $\mathcal{X}_{\mu}$ et $\mathcal{X}_{\leqslant \mu}$ dont les points $k$-rationnels sont respectivement :

$$
\mathcal{L}_{\mu}=\left\{\begin{array}{c|c}
\text { réseaux } L \text { de } M & \text { il existe une base } m_{1}, \ldots, m_{d} \text { de } L \text { telle que } \\
u^{\mu_{1}} m_{1}, \ldots, u^{\mu_{d}} m_{d} \text { soit une base de } \sigma\left(k\left[\left[u^{1 / b}\right]\right] \otimes_{k[[u]]} L\right)
\end{array}\right\}
$$

et

$$
\mathcal{L}_{\leqslant \mu}=\bigcup_{\mu^{\prime} \leqslant \mu} \mathcal{L}_{\mu^{\prime}}
$$

où l'on convient que $\mu^{\prime}=\left(\mu_{1}^{\prime}, \cdots, \mu_{d}^{\prime}\right)$ est plus petit ou égal à $\mu$ si $\mu_{1}^{\prime}+\cdots+\mu_{t}^{\prime} \leqslant \mu_{1}+\cdots+\mu_{t}$ pour tout $t \in\{1, \ldots, d\}$ avec égalité si $t=d$. Dans cet article, nous nous intéressons également à la dimension de ces variétés. Pour énoncer les résultats obtenus, il est commode de munir $\mathbb{R}^{d}$ du produit scalaire usuel $\langle\cdot \mid \cdot\rangle_{d}$ et d'introduire le vecteur

$$
\vec{\rho}=\left(\frac{d-1}{2}, \frac{d-3}{2}, \ldots, \frac{1-d}{2}\right) \in \mathbb{R}^{d}
$$

(la $i$-ième coordonnée est donnée par la formule $\frac{d+1}{2}-i$ ).

Définition 3. On dit qu'un $d$-uplet $\mu=\left(\mu_{1}, \ldots, \mu_{d}\right) \in \mathbb{R}^{d}$ est :

- b-régulier si $\mu_{i}-\mu_{i+1} \leqslant b\left(\mu_{d-i}-\mu_{d-i+1}\right)$ pour tout $i \in\{1, \ldots, d-1\}$;

- intégralement b-régulier s'il est $b$-régulier, si tous les $\mu_{i}$ sont entiers et $b-1$ divise $\mu_{1}+\cdots+\mu_{d}$,

- fortement intégralement b-régulier s'il est intégralement $b$-régulier et vérifie en plus :

$$
\mu_{d-1}-\mu_{d} \leqslant b\left(\mu_{1}-\mu_{2}\right)-d\left(b^{2}-1\right) .
$$

Les définitions d'éléments $b$-réguliers et intégralement $b$-réguliers semblent s'imposer dans ce contexte. Par contre, l'inégalité renforcée qui apparaît dans la définition de fortement intégralement $b$-régulier n'est probablement pas optimale et devra sans doute être corrigée ultérieurement. On remarque néanmoins que si $\mu=\left(\mu_{1}, \ldots, \mu_{d}\right)$ est $b$-régulier, alors $\mu_{1} \geqslant \cdots \geqslant \mu_{d}$. Réciproquement si les $\mu_{i}$ sont rangés par ordre décroissant et deux à deux distincts, le $d$-uplet $\mu$ est $b$-régulier pour $b$ suffisamment grand.

Théorème 4. Soit $\mu=\left(\mu_{1}, \ldots, \mu_{d}\right) \in \mathbb{Z}^{d}$ tel que $\mu_{1} \geqslant \mu_{2} \geqslant \cdots \geqslant \mu_{d}$. Si b-1 ne divise pas $\mu_{1}+\cdots+\mu_{d}$, alors la variété $\mathcal{X}_{\mu}$ est vide. On suppose donc tout au long du théorème que $b-1$ divise $\mu_{1}+\cdots+\mu_{d}$.

On pose $\varepsilon=1$ si $h=0$ et $\varepsilon=0$ dans le cas contraire. Alors, il existe un entier $\delta \in\left\{0,1, \ldots, \varepsilon \cdot \frac{d(d-1)}{2}\right\}$ tel que l'on ait la congruence :

$$
\operatorname{dim}_{k} \mathcal{X}_{\mu} \equiv \delta-\sum_{i=1}^{d} i \cdot \mu_{i} \quad(\bmod b-1)
$$

En particulier, si $h \geqslant 0$, on $a$ :

$$
\operatorname{dim}_{k} \mathcal{X}_{\mu} \equiv-\sum_{i=1}^{d} i \cdot \mu_{i} \quad(\bmod b-1)
$$

On suppose maintenant en plus $b \geqslant 1+\left[\frac{(d-1)^{2}}{4}\right]$. Alors on $a$ :

$$
\operatorname{dim}_{k} \mathcal{X}_{\mu} \leqslant \varepsilon \cdot \frac{d(d-1)}{2}+(b-1) \cdot \min _{w \in \mathfrak{S}_{d}} \sum_{i=1}^{d} \sum_{n=1}^{\infty} \mu_{i} \cdot \frac{d+1-i-w^{n}(i)}{b^{n}}
$$

où, bien entendu, $\mathfrak{S}_{d}$ désigne le groupe des permutations de $\{1, \ldots, d\}$ et $w^{n}=w \circ \cdots \circ w$ ( $n$ fois). En outre, si $\mu$ est b-régulier, alors le minimum précédent est atteint pour $w=w_{0}: i \mapsto d+1-i$ et vaut $\frac{1}{b^{2}-1} \cdot\langle 2 \vec{\rho} \mid \mu\rangle_{d}$ (le produit de ce minimum par $(b-1)$ est donc égal à $\left.\frac{1}{b+1} \cdot\langle 2 \vec{\rho} \mid \mu\rangle_{d}\right)$. 
On suppose toujours $b \geqslant 1+\left[\frac{(d-1)^{2}}{4}\right]$. Il existe des constantes positives $c_{1}$ et $c_{2}$ (qui ne dépendent que de $d$ et b) telles que si les $\mu_{i}$ vérifient en plus $\mu_{i} \geqslant \mu_{i+1}+c_{1}$ pour tout $i$, alors :

$$
\operatorname{dim}_{k} \mathcal{X}_{\mu} \geqslant-c_{2}+(b-1) \cdot \min _{w \in \mathfrak{S}_{d}} \sum_{i=1}^{d} \sum_{n=1}^{\infty} \mu_{i} \cdot \frac{d+1-i-w^{n}(i)}{b^{n}} .
$$

Encore une fois, on ne dit rien quant à l'équidimensionnalité des variétés $\mathcal{X}_{\mu}$. Cependant, lorsque $h \neq 0$, on peut se demander s'il est vrai que toutes les composantes irréductibles de $\mathcal{X}_{\mu}$ ont des dimensions congrues à $-\sum_{i=1}^{d} i \cdot \mu_{i}$ modulo $(b-1)$. À part cela, il est clair que les sommes infinies qui apparaissent dans la formule du théorème précédent convergent. Étant donné que toute permutation $w$ est d'ordre fini, on peut même facilement calculer leur limite qui s'exprime toujours comme le produit de $\mu_{i}$ par un nombre rationnel, ce dernier étant même la valeur en $b$ d'une fraction rationnelle à coefficients entiers.

On en vient maintenant aux variétés $\mathcal{X}_{\leqslant \mu}$.

Théorème 5. Soit $\mu=\left(\mu_{1}, \ldots, \mu_{d}\right) \in \mathbb{R}^{d}$ tel que $\mu_{1} \geqslant \mu_{2} \geqslant \cdots \geqslant \mu_{d}$. On pose $\varepsilon=1$ si $h=0$ et $\varepsilon=0$ dans le cas contraire. Alors :

$$
-(d-1)^{2}-\frac{(d-2)^{2}}{4}+\sup _{\substack{\mu^{\prime} \leqslant \mu \\ \mu^{\prime} \text { f.i. } b \text {-rég. }}} \frac{\left\langle 2 \vec{\rho} \mid \mu^{\prime}\right\rangle_{d}}{b+1} \leqslant \operatorname{dim}_{k} \mathcal{X}_{\leqslant \mu} \leqslant \varepsilon \cdot \frac{d(d-1)}{2}+\frac{\langle 2 \vec{\rho} \mid \mu\rangle_{d}}{b+1} .
$$

Si, en outre, $b \geqslant 1+\max \left(d,\left[\frac{(d-1)^{2}}{4}\right]\right)$, alors la majoration peut être renforcée comme suit :

$$
\operatorname{dim}_{k} \mathcal{X}_{\leqslant \mu} \leqslant \varepsilon \cdot \frac{d(d-1)}{2}+\sup _{\substack{\mu^{\prime} \leqslant \mu \\ \mu^{\prime} b \text {-rég. }}} \frac{\left\langle 2 \vec{\rho} \mid \mu^{\prime}\right\rangle_{d}}{b+1}
$$

Il est utile de commenter un peu le théorème. Pour la première assertion, on remarque que si $\mu$ est luimême fortement intégralement $b$-régulier, alors la borne supérieure qui apparaît est atteinte pour $\mu^{\prime}=\mu$. Ainsi le théorème dit, dans ce cas, que la quantité $\frac{\langle 2 \vec{\rho} \mid \mu\rangle_{d}}{b+1}$ est une bonne approximation de la dimension de $\mathcal{X}_{\leqslant \mu}$. La deuxième assertion mérite, quant à elle, une discussion plus approfondie. Tout d'abord, il est facile de prouver que la borne supérieure qui apparaît est plus petite ou égale — et en général strictement plus petite, du moins si $\mu$ n'est pas lui-même $b$-régulier — que $\frac{\langle 2 \vec{\rho} \mid \mu\rangle_{d}}{b+1}$; ainsi, comme cela est déjà précisé dans l'énoncé du théorème, la majoration écrite est meilleure que la précédente. Par ailleurs, on a clairement $\mathcal{X}_{\leqslant \mu}=\bigcup_{\mu^{\prime} \leqslant \mu} \mathcal{X}_{\leqslant \mu^{\prime}}$, d'où on déduit que :

$$
\operatorname{dim}_{k} \mathcal{X}_{\leqslant \mu}=\sup _{\mu^{\prime} \leqslant \mu} \operatorname{dim}_{k} \mathcal{X}_{\leqslant \mu^{\prime}}
$$

L'inégalité du théorème dit donc en substance que, si $b \geqslant 1+\max \left(d,\left[\frac{(d-1)^{2}}{4}\right]\right)$, les variétés $\mathcal{X}_{\leqslant \mu^{\prime}}$, pour $\mu^{\prime} \leqslant$ $\mu$ non $b$-régulier, n' apportent pratiquement pas de nouvelles dimensions à $\mathcal{X}_{\leqslant \mu}$. Notamment, contrairement à ce qui se passe dans le cas des variétés de Deligne-Lusztig affines, il n'est pas clair — et ce n'est d'ailleurs en général pas vrai — que l'essentiel de la dimension de $\mathcal{X}_{\leqslant \mu}$ est concentré dans la variété $\mathcal{X}_{\mu}$. Du fait que $\mathcal{X}_{\mu}$ est un ouvert dans $\mathcal{X}_{\leqslant \mu}$, il suit que $\mathcal{X}_{\leqslant \mu}$ n'est généralement pas équidimensionnelle lorsque $\mu$ n'est pas $b$-régulier.

Finalement, la borne supérieure qui apparaît dans la dernière inégalité du théorème est aussi égale au minimum d'un nombre fini de formes linéaires sur $\mathbb{R}^{d}$, ce qui permet de la calculer efficacement. Malgré tout, bien que ces formes linéaires soient définies de façon plutôt explicite, leur nombre et leur complexité croît très rapidement lorsque $d$ augmente. À titre d'exemple, le tableau回 (page 46 ) les donne pour $d \leqslant 4$.

Il est vrai que les théorèmes précédents peuvent sembler ni vraiment intéressants, ni faciles à appliquer car ils énoncent finalement, sous des hypothèses plutôt fortes, des résultats à la fois techniques et imprécis. Ce point de vue convient toutefois d'être nuancé (voire reconsidéré) pour plusieurs raisons.

Tout d'abord, en ce qui concerne les hypothèses, il ne faut pas voir les résultats de cet article comme une fin en soi, mais bel et bien comme un premier pas vers la résolution d'un problème plus général. Mieux encore, le théorème $\bigoplus$ semble directement donner les clés de cette vaste généralisation. Généralisation tout d'abord au cas d'un opérateur $\sigma: M \rightarrow M$ n'agissant pas nécessairement coordonnée par coordonnée 
(c'est-à-dire d'une représentation $V_{\mathbb{F}}$ quelconque avec encore $\mathbb{F}=\mathbb{F}_{p}$ ) pour lequel l'auteur pense que le théorème 4 s'étend simplement en modifiant certaines constantes (voir conjecture 4.5, page 51, pour plus de précisions). Mais généralisation également au cas des variétés de Kisin associés à un groupe réductif connexe déployé quelconque (le cas présenté ici est celui de $\mathrm{GL}_{d}$ ), ce qui englobe notamment le cas des variétés de Kisin associées à des représentations $V_{\mathbb{F}}$ à coefficients dans une extension finie arbitraire de $\mathbb{F}_{p}$. Pour un énoncé précis dans cette direction, on se contente de renvoyer le lecteur au $\$ 4.2 .3$, et plus particulièrement à la conjecture 4.7. De surcroît, l'auteur pense que les méthodes développées dans cet article sont de nature à s'étendre à la situation générale des groupes réductifs, et y reviendra sans doute dans un travail ultérieur.

Au sujet, ensuite, de la technicité des résultats, il est à noter que, si l'on se restreint à des $\mu$ intégralement $b$-réguliers, tous les théorèmes de cet article deviennent particulièrement simples puisqu'alors, à une constante près, tous les minorants et majorants sont égaux à $\frac{1}{b+1} \cdot\langle 2 \vec{\rho} \mid \mu\rangle_{d}$. Il est vrai, enfin, que le problème de l'imprécision reste, quant à lui, non résolu même conjecturalement. Il est toutefois intéressant de comparer la forme générale des formules du théorème 4 avec les formules connues pour les dimensions des variétés de Deligne-Lusztig, démontrées dans [4] et [16]. Un rapide coup d'œil à ces références montre que cette dimension s'écrit comme le somme d'une contribution linéaire (qui s'exprime en terme de produit scalaire avec le vecteur $\rho$ comme dans cet article) et d'une contribution bornée. Il semble donc que l'on ait découvert, ici, l'analogue de la partie linéaire et, en ce sens, le théorème 7 apparaît à nouveau comme un premier pas incontournable pour le calcul de la dimension des variétés de Kisin en toute généralité.

Présentation sommaire de la méthode et du plan de l'article De façon générale, la méthode suivie est largement inspirée de l'article [16] de Viehmann : l'idée est de définir une stratification plus fine des variétés de Kisin pour laquelle on sait calculer précisément la dimension des strates. Le problème du calcul de la dimension des variétés de Kisin se métamorphose alors complètement en un nouveau problème combinatoire que l'on parvient à résoudre ensuite, au moins de façon approchée. Si la première partie du cheminement suit d'assez près les arguments de Viehmann, les chemins se séparent nettement pour la résolution du problème combinatoire qui s'avère être bien plus délicat dans le cas des variétés de Kisin.

De façon plus détaillée, on commence dans le $\$ 1.1 .1$ par associer à chaque réseau $L \subset M$ une donnée combinatoire $\varphi(L)$ constituée de $d$ fonctions. Il résulte des travaux de Viehmann que ces données combinatoires sont soumises à des nombreuses contraintes qui imposent une forte rigidité. Dans le $\S 1.2$, on étudie plus en détails ces contraintes, et on en déduit une paramétrisation des « données combinatoires admissibles » par les points d'un réseau à l'intérieur d'un convexe vivant dans un espace vectoriel réel de dimension $\frac{d(d+1)}{2}$. D'un point de vue géométrique, la construction précédente définit une stratification des variétés de Kisin par des sous-espaces localement fermés $\mathcal{X}_{\varphi}$. On démontre alors le théorème 1.18 qui donne une estimation (et même une formule exacte dans certains cas) pour la dimension des variétés $\mathcal{X}_{\varphi}$, qui s'exprime de façon complètement explicite en fonction du point du réseau paramétrant $\varphi$.

À ce stable, le problème du calcul des variétés de Kisin se reformule complètement en termes de programmation linéaire. Dans le $\$ 2$, on introduit les outils nécessaires (qui sont plus ou moins classiques) à sa résolution et, à titre d'exemple, on fait fonctionner la méthode dans un cas simple, aboutissant ainsi à une démonstration de la majoration du théorème 2 sous l'hypothèse supplémentaire $b>d$. Dans le $\$ 3$, la machine se met enfin véritablement en route avec pour objectif de démontrer complètement les théorèmes 2. 4 et 5. Cela devient alors assez vite très technique et il n'est pas vraiment envisageable d'en dire beaucoup plus dans cette introduction, sauf peut-être que l'essentiel de la démonstration consiste à donner des descriptions précises de certaines parties convexes de $\mathbb{R}^{d}$, et ne fait intervenir que des arguments élémentaires (mais parfois subtils).

Dans le $\S$ finalement sont examinées plusieurs perspectives offertes par les résultats des théorèmes 2 . 4 et 5 , et notamment les généralisations éventuelles à des $\sigma$ agissant sur $M$ pas nécessairement coordonnée par coordonnée (voir $\$ 4.2 .1$ ) et d'autres groupes réductifs (voir $\$ 4.2 .3$ ) qui ont été évoquées précédemment. On discute également, dans le $\$ 4.1$, de la possibilité d'obtenir une formule exacte pour la dimension. À part un calcul explcite en dimension 3 dont la conclusion reste mystérieuse, il faut bien dire que rien de vraiment précis ne se dégage pour l'instant.

Remerciements C'est un plaisir de remercier Michael Rapoport pour m'avoir soumis le problème dont il est question dans cet article, et également pour ses encouragements constants. Je le remercie également de m'avoir plusieurs fois invité à l'Université de Bonn, qui est un lieu extraordinaire pour faire des mathéma- 
tiques. Mes remerciements vont aussi à Eugen Hellmann pour d'intéressantes discussions et à Eva Viehmann pour m'avoir fait connaître son article [16], duquel tout ce travail est inspiré. Je remercie également David Monniaux pour m'avoir fait connaître son logiciel mjollnir [12] qui m'a été fort utile lors de l'élaboration de cet article.

Finalement, je remercie l'Agence Nationale de la Recherche (ANR) pour son soutien financier par l'intermédiaire du projet CETHop (Calculs Effectifs en Théorie de Hodge $p$-adique) référencé ANR-09JCJC-0048-01.

\section{Une stratification utile}

On commence par rappeler très brièvement que les variétés $\mathcal{X}_{\leqslant e}, \mathcal{X}_{\mu}$ et $\mathcal{X}_{\leqslant \mu}$ sont définies par l'intermédiaire de leur $\ll$ foncteur des points $\gg$. Pour $\mathcal{X}_{\leqslant e}$ par exemple, étant donné $R$ une $k$-algèbre, on définit l'ensemble $\mathcal{X}_{\leqslant e}(R)$ comme l'ensemble des $R[[u]]$-sous-modules $L$ de $M \otimes_{k((u))} R((u))=R((u))^{d}$ qui sont tels que :

i) $L$ est un réseau de $M \otimes_{k((u))} R((u))=R((u))^{d}$, c'est-à-dire que $L$ est un $R[[u]]$-module localement libre de type fini (pour la topologie de Spec $R$ ) et le morphisme naturel $L \otimes_{R[[u]]} R((u)) \rightarrow M$ est un isomorphisme;

ii) la condition (11) est satisfaite où $\phi$ est remplacé par $\sigma$ (et où $\sigma$ opère encore par $x \mapsto x^{p^{h}}$ sur $R$, envoie $u$ sur $u^{b}$ et s'étend à $M \otimes_{k((u))} R((u))=R((u))^{d}$ en agissant coordonnée par coordonnée). On montre ensuite que le foncteur $R \mapsto \mathcal{X}_{\leqslant e}(R)$ ainsi défini est représentable par un schéma de type fini sur $k$ qui apparaît naturellement comme un sous-schéma fermé de la grassmanienne affine sur $k$. Des considérations analogues conduisent à une définition rigoureuse de $\mathcal{X}_{\mu}$ et $\mathcal{X}_{\leqslant \mu}$.

Le but de ce premier chapitre est de montrer que les variétés précédentes sont stratifiées par des sousvariétés $\mathcal{X}_{\varphi}$ (où $\varphi$ est une donnée combinatoire qui dépend de $\frac{d(d+1)}{2}$ entiers) dont on sait calculer la dimension.

\subsection{Donnée combinatoire associée à un réseau}

Dans cette partie, on associe à chaque réseau $L$ de $M$ la donnée combinatoire évoquée précédemment qui s'avère être un ensemble de $d$ fonctions soumises à un certain nombre de contraintes. On montre ensuite que ces contraintes imposent une rigidité telle qu'elles réduisent la donnée des $d$ fonctions à celle de seulement $\frac{d(d+1)}{2}$ nombres.

\subsubsection{Définitions}

Soit val la valuation naturelle sur $k((u))$ : la valuation d'une somme $\sum_{i \gg-\infty} a_{i} u^{i}$ est le plus petit entier $v$ tel que $a_{v} \neq 0$ et on convient que $\operatorname{val}(0)=+\infty$. La valuation s'étend de manière unique à l'extension totalement ramifiée $k\left(\left(u^{1 / b}\right)\right)$, et on note encore val ce prolongement; on a donc $\operatorname{val}\left(u^{1 / b}\right)=\frac{1}{b}$.

On pose $M_{k\left(\left(u^{1 / b}\right)\right)}=k\left(\left(u^{1 / b}\right)\right) \otimes_{k((u))} M=k\left(\left(u^{1 / b}\right)\right)^{d}$ et on note $\left(e_{1}, \ldots, e_{d}\right)$ la base canonique de $M=k((u))^{d}$. Les vecteurs $1 \otimes e_{i}$ forment une base de $M_{k\left(\left(u^{1 / b}\right)\right)}$ sur le corps $k\left(\left(u^{1 / b}\right)\right)$. La valuation val définit une application $\operatorname{val}_{M}: M_{k\left(\left(u^{1 / b}\right)\right)} \backslash\{0\} \rightarrow \frac{1}{b} \mathbb{Z} \times\{1, \ldots, d\}$ par la formule :

$$
\begin{aligned}
& \operatorname{val}_{M}\left(x_{1}, \ldots, x_{d}\right)=(v, i) \quad \text { où } v=\min \left\{\operatorname{val}\left(x_{1}\right), \ldots, \operatorname{val}\left(x_{d}\right)\right\} \\
& \text { et } \quad i=\min \left\{j \mid v=\operatorname{val}\left(x_{j}\right)\right\}
\end{aligned}
$$

On prolonge $\operatorname{val}_{M}$ à $M_{k\left(\left(u^{1 / b}\right)\right)}$ tout entier en convenant que val $\operatorname{val}_{M}(0)=\infty$ où le symbole $\infty$ désigne un nouvel élément que l'on ajoute au produit $\frac{1}{b} \mathbb{Z} \times\{1, \ldots, d\}$. On vérifie immédiatement que si $\lambda \in k\left(\left(u^{1 / b}\right)\right)$ et $x \in M_{k\left(\left(u^{1 / b}\right)\right)}$, on a $\operatorname{val}_{M}(\lambda x)=\operatorname{val}(\lambda)+\operatorname{val}_{M}(x)$ avec la convention évidente que $\infty+t=\infty$ lorsque $t$ est un nombre rationnel ou un couple $(v, i) \in \frac{1}{b} \mathbb{Z} \times\{1, \ldots, d\}$. De plus, si l'on munit l'ensemble $\frac{1}{b} \mathbb{Z} \times\{1, \ldots, d\}$ de l'ordre lexicographique et que l'on convient que $\infty$ est strictement plus grand que tous les couples $(v, i)$, alors, pour tous $x$ et $y$ dans $M$, on a $\operatorname{val}_{M}(x+y) \geqslant \min \left\{\operatorname{val}_{M}(x), \operatorname{val}_{M}(y)\right\}$ et l'égalité a lieu dès que $\operatorname{val}_{M}(x) \neq \operatorname{val}_{M}(y)$.

2. Cela signifie que $(v, i)<\left(v^{\prime}, i^{\prime}\right)$ si, et seulement si soit $v<v^{\prime}$, soit $v=v^{\prime}$ et $i<i^{\prime}$. 
Définition 1.1. Soit $L$ un réseau de $M$. Pour tout $v \in \frac{1}{b} \mathbb{Z}$ et tout $i \in\{1, \ldots, d\}$, on pose

$$
\tilde{\varphi}_{i}(L)(v)=\sup _{\substack{x \in k\left[\left[u^{1 / b}\right]\right] \otimes_{k[[u]]} L \\ \operatorname{val}_{M}(x)=(v, i)}}\left(\sup \left\{n \in \mathbb{Z} \mid \sigma(x) \in u^{n} L\right\}\right)
$$

où, par convention, la borne supérieure d'un ensemble non majoré est $+\infty$ et celle de l'ensemble vide est $-\infty$.

La définition ci-dessus n'est en fait rien d'autre qu'une adaptation de la définition des fonctions $\varphi$ qui apparaissent dans [16]. On a choisi de conserver la formulation de loc. cit. mais il est sans doute bon de garder à l'esprit que l'on peut interpréter les nombres $\tilde{\varphi}_{i}(L)(v)$ de façon plus parlante en termes de distance. Pour ce faire, on munit l'espace $k((u))$ de la norme $\|x\|=a^{\operatorname{val}(x)}$ pour un certain réel $a$ fixé dans $] 0,1[$. Le choix du réseau $L$ définit une norme $\|\cdot\|_{L}$ sur $M$ comme suit : si $\left(f_{1}, \ldots, f_{d}\right)$ est une $k[[u]]$-base de $L$, on pose $\left\|\sum_{i=1}^{d} \lambda_{i} f_{i}\right\|_{L}=\max \left\|\lambda_{i}\right\|$. La norme obtenue ne dépend alors pas du choix des $f_{i}$. Par ailleurs, un examen direct des définitions montre que, pour la distance associée à $\|\cdot\|_{L}$, le nombre réel $a^{\tilde{\varphi}_{i}(L)(v)}$ est égal à la distance de l'origine au sous espace $\sigma(B)$ où $B$ est l'ensemble des éléments de $k\left[\left[u^{1 / b}\right]\right] \otimes_{k[[u]]} L$ de valuation $(v, i)$ (ensemble que l'on peut voir aussi comme la boule unité pour une autre norme). Comme $0 \notin \sigma(B)$ (puisque $\sigma$ est injectif et que 0 n'est pas dans $B$ ), il suit de la description précédente que la fonction $\tilde{\varphi}_{i}(L)$ ne prend jamais la valeur $+\infty$.

Proposition 1.2. Soit $L$ un réseau de $M$. Les fonctions $\tilde{\varphi}_{i}(L): \frac{1}{b} \mathbb{Z} \rightarrow \mathbb{Z} \cup\{-\infty\}, v \mapsto \tilde{\varphi}_{i}(L)(v)$ vérifient les propriétés suivantes.

1. Pour tout $i$, la fonction $\tilde{\varphi}_{i}(L)$ est strictement croissante où, par un léger abus d'écriture, l'on entend par là que $\tilde{\varphi}_{i}(L)$ est croissante et qu'elle est strictement croissante sur l'ensemble où elle prend des valeurs finies.

2. Pour tout $i$, il existe un entier $\tilde{q}_{i}(L)$ tel que

- la fonction $\tilde{\varphi}_{i}(L)$ prend des valeurs finies exactement sur l'intervalle $\left[\tilde{q}_{i}(L),+\infty[\right.$, et

- pour $v$ suffisamment grand, on a $\tilde{\varphi}_{i}(L)(v)=b v-\tilde{q}_{i}(L)$.

3. Pour $j \in\{1, \ldots, d\}$, il existe des fonctions croissantes $\psi_{j}(L): \mathbb{Z} \rightarrow \frac{1}{b} \mathbb{Z} \cup\{-\infty\}$ telles que $\psi_{1}(L) \leqslant$ $\psi_{2}(L) \leqslant \cdots \leqslant \psi_{d}(L)$ et pour tout couple $(v, \mu) \in \frac{1}{b} \mathbb{Z} \times \mathbb{Z}$, il y a autant d'indices $i \in\{1, \ldots, d\}$ tels que $\mu=\tilde{\varphi}_{i}(L)(v)$ que d'indices $j \in\{1, \ldots, d\}$ tels que $v=\psi_{j}(L)(\mu)$.

Ces fonctions sont en outre uniquement déterminées.

4. Si $u^{\mu_{1}(L)}, \ldots, u^{\mu_{d}(L)}$, avec $\mu_{1}(L) \geqslant \cdots \geqslant \mu_{d}(L)$, sont les diviseurs élémentaires $d u k[[u]]$-module engendré par $\sigma(L)$ par rapport à $L$, alors, pour tout $i$, la fonction $\psi_{i}$ prend des valeurs finies exactement sur l'intervalle $\left[\mu_{i}(L),+\infty[\right.$.

Démonstration. C'est simplement une transposition du lemme 4.1 de [16].

Si $L$ est un réseau de $M$, on définit aussi des fonctions $\varphi_{1}(L), \ldots, \varphi_{d}(L): \frac{1}{b} \mathbb{Z} \rightarrow \mathbb{Z} \cup\{-\infty\}$ en convenant que pour tout $v \in \frac{1}{b} \mathbb{Z}$, les nombres $\varphi_{1}(L)(v), \ldots, \varphi_{d}(L)(v)$ sont les mêmes que $\tilde{\varphi}_{1}(L)(v), \ldots, \tilde{\varphi}_{d}(L)(v)$ mais triés par ordre décroissant. Les fonctions précédentes vérifient donc tautologiquement l'inégalité $\varphi_{1}(L) \geqslant \varphi_{2}(L) \geqslant \cdots \geqslant \varphi_{d}(L)$ et on montre sans peine qu'elles satisfont encore aux quatre alinéas de la proposition 1.2 : les entiers $\mu_{j}(L)$ restent inchangés tandis que les $\tilde{q}_{i}(L)$ sont a priori permutés. Dans la suite, l'entier correspondant à la fonction $\varphi_{i}(L)$ sera noté $q_{i}(L)$.

\subsubsection{Un exemple en dimension 2}

En guise d'illustration de la proposition 1.2 et pour familiariser le lecteur avec la définition 1.1, on examine un exemple en dimension 2 (i.e. avec $d=2$ ). Si $L$ est un réseau de $M$, il existe des entiers relatifs $\alpha, \delta$ et un élément $c \in k((u))$ de valuation $\gamma$ tels que $L$ soit engendré sur $k[[u]]$ par les vecteurs $f_{1}=\left(u^{\alpha}, 0\right)$ et $f_{2}=\left(c, u^{\delta}\right)$. De plus, quitte à retirer à $c$ un multiple de $u^{\alpha}$, on peut supposer que soit $c=0$, soit $\gamma<\alpha$.

Lemme 1.3. Soit $x=\left(x_{1}, x_{2}\right) \in k\left(\left(u^{1 / b}\right)\right) \otimes_{k((u))} M$. Le plus grand $n$ tel que $x \in u^{n} k\left[\left[u^{1 / b}\right]\right] \otimes_{k[[u]]} L$ est le plus petit des deux nombres $\operatorname{val}\left(x_{2}\right)-\delta$ et $\operatorname{val}\left(x_{1}-x_{2} c u^{-\delta}\right)-\alpha$.

3. Et ce sera aussi le cas dans tout l'article. 
Démonstration. Le vecteur $\left(x_{1}, x_{2}\right)$ se décompose sur la base $\left(f_{1}, f_{2}\right)$ sous la forme :

$$
\left(x_{1}, x_{2}\right)=\left(x_{1} u^{-\alpha}-x_{2} c u^{-(\alpha+\delta)}\right) f_{1}+x_{2} u^{-\delta} f_{2} .
$$

Ainsi il appartient à $M$ si, et seulement si les deux coefficients que l'on voit apparaître dans l'écriture précédente sont de valuation positive ou nulle, c'est-à-dire si, et seulement si val $\left(x_{1}-x_{2} c u^{-\delta}\right) \geqslant \alpha$ et $\operatorname{val}\left(x_{2}\right) \geqslant \delta$. Le lemme en découle.

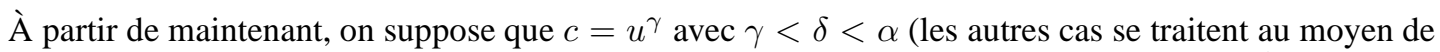
calculs analogues). On détermine tout d'abord la fonction $\tilde{\varphi}_{1}(L)$. Soient $v \in \frac{1}{b} \mathbb{Z}$ et $x \in k\left[\left[u^{1 / b}\right]\right] \otimes_{k[[u]]} L$ tel que val $(x)=(v, 1)$. Quitte à multiplier $x$ par un élément inversible de $k\left[\left[u^{1 / b}\right]\right]$, ce qui ne change pas la valeur de la deuxième borne inférieure dans la formule (1.1), on peut supposer que $x$ s'écrit $u^{v} \otimes e_{1}+u^{v} y \otimes e_{2}$ où $y \in k\left[\left[u^{1 / b}\right]\right]$. Par le lemme 1.3 , le fait que $x$ appartienne à $L$ se traduit par les inégalités $\operatorname{val}\left(u^{v} y\right) \geqslant \delta$ et $\operatorname{val}\left(u^{v}-u^{v+\gamma-\delta} y\right) \geqslant \alpha$, ce qui se réécrit encore :

$$
\operatorname{val}(y) \geqslant \delta-v \quad \text { et } \quad y \equiv u^{\delta-\gamma} \quad\left(\bmod u^{\delta-\gamma+(\alpha-v)}\right)
$$

On suppose pour commencer que $v<\alpha$. Dans ce cas, la dernière congruence implique que $y$ est de valuation $\delta-\gamma$, d'où on déduit $\delta-\gamma \geqslant \delta-v$, c'est-à-dire $v \geqslant \gamma$. Autrement dit, si $v<\gamma$, aucun $x$ ne satisfait aux conditions requises et on a alors $\tilde{\varphi}_{1}(L)(v)=-\infty$. Si, au contraire, $\gamma \leqslant v<\alpha$, il existe des $x$ convenables qui sont précisément les vecteurs de la forme

$$
x=u^{v} \otimes e_{1}+u^{v+\delta-\gamma}\left(1+u^{\alpha-v} z\right) \otimes e_{2}
$$

pour un certain élément $z \in k\left[\left[u^{1 / b}\right]\right]$. Le lemme 1.3 appliqué au vecteur $\sigma(x)$ donne ainsi :

$$
\begin{aligned}
\tilde{\varphi}_{1}(L)(v) & =\sup _{z \in k\left[\left[u^{1 / b}\right]\right]}\left(\min \left\{b(v+\delta-\gamma)-\delta, b v-\alpha+\operatorname{val}\left(1-u^{(b-1)(\delta-\gamma)}\left(1+u^{b(\alpha-v)} \sigma(z)\right)\right)\right\}\right) \\
& =\sup _{z \in k\left[\left[u^{1 / b}\right]\right]}(\min \{b(v+\delta-\gamma)-\delta, b v-\alpha\}) \quad \operatorname{car} \delta-\gamma>0 \\
& =\min \{b(v+\delta-\gamma)-\delta, b v-\alpha\}
\end{aligned}
$$

Or, $b(\delta-\gamma)>0>\delta-\alpha$, d'où on obtient finalement $\tilde{\varphi}_{1}(L)(v)=b v-\alpha$ pour $v \in[\gamma, \alpha[$.

On suppose désormais que $v \geqslant \alpha$. Dans ce cas, la première condition de la ligne (1.2) est automatique vérifiée (on rappelle que $y$ est dans $k\left[\left[u^{1 / b}\right]\right]$ ) tandis que la congruence qui suit se réduit à val $(y) \geqslant \delta-\gamma+$ $\alpha-v$. On est ainsi amené à calculer

$$
\sup _{y}\left(\min \left\{b(v+\operatorname{val}(y))-\delta, b v-\alpha+\operatorname{val}\left(1-u^{\gamma-\delta} \sigma(y)\right)\right\}\right)
$$

où la borne supérieure est prise sur tous les $y$ dont la valuation est à la fois supérieure ou égale à 0 et à $\delta-\gamma+\alpha-v$. Pour les $y$ de valuation strictement plus petite (resp. strictement plus grande) que $\frac{\delta-\gamma}{b}$, la valuation de la différence $1-u^{\gamma-\delta} \sigma(y)$ vaut $\gamma-\delta+b \operatorname{val}(y)$ (resp. 0 ), et un calcul simple montre que le minimum qui apparaît dans la formule précédente est inférieur ou égal (resp. est égal) à $b v-\alpha$. Par contre, pour les $y$ qui ont la valuation critique $\frac{\delta-\gamma}{b}$, la borne supérieure est atteinte lorsque $1-u^{\gamma-\delta} \sigma(y)$ s'annule, et elle est égale à $b(v+\operatorname{val}(y))-\delta=b v-\gamma$. Du fait que $\gamma<\alpha$, on déduit que la valeur de $\tilde{\varphi}_{1}(L)(v)$ est $b v-\gamma$ dès que l'on peut choisir $y$ de valuation $\frac{\delta-\gamma}{b}$, c'est-à-dire dès que $\frac{\delta-\gamma}{b} \geqslant \delta-\gamma+\alpha-v$ ou encore après simplification $v \geqslant \alpha+\frac{b-1}{b}(\delta-\gamma)$, et qu'elle est $b v-\alpha$ dans le cas contraire.

En résumé, la fonction $\tilde{\varphi}_{1}(L)$ prend la forme simple suivante :

$$
\begin{aligned}
\tilde{\varphi}_{1}(L): \quad v & \mapsto-\infty \quad \text { si } v<\gamma \\
v & \mapsto b v-\alpha \quad \text { si } \gamma \leqslant v<\alpha+\frac{b-1}{b}(\delta-\gamma) \\
v & \mapsto b v-\gamma \quad \text { sinon. }
\end{aligned}
$$

On en vient à la fonction $\tilde{\varphi}_{2}(L)$. Soit $x \in L$ tel que val $M(x)=(v, 2)$. Comme dans le cas précédent, quitte à multiplier $x$ par un élément inversible de $k\left[\left[u^{1 / b}\right]\right]$, on peut supposer qu'il est de la forme $x=$ $u^{v} y \otimes e_{1}+u^{v} \otimes e_{2}$ où $y \in k\left(\left(u^{1 / b}\right)\right)$ est un élément de valuation strictement positive. Comme l'on sait que $x$ est élément de $L$, le lemme 1.3 implique que val $\left(y-u^{\gamma-\delta}\right) \geqslant \alpha-v$, soit encore $\gamma-\delta \geqslant \alpha-v$ car 
$\gamma-\delta<0<\operatorname{val}(y)$. Ainsi la valuation de la différence $y-u^{\gamma-\delta}$ est $\gamma-\delta$. En particulier, si $v<\alpha+\delta-\gamma$ aucun élément $y$ ne satisfait aux conditions requises, et donc $\tilde{\varphi}_{2}(L)(v)=-\infty$. Par contre, si $v \geqslant \alpha+\delta-\gamma$, tous les $y$ de valuation strictement positive conviennent et un calcul analogue à celui mené pour $\tilde{\varphi}_{1}(L)$ conduit dans ce cas à $\tilde{\varphi}_{2}(L)(v)=b v-\alpha+\gamma-\delta$. En résumé, on a donc :

$$
\begin{array}{rlrl}
\tilde{\varphi}_{2}(L): & v & \mapsto & \text { si } v<\alpha+\delta-\gamma \\
v & \mapsto b v-\alpha+\gamma-\delta & \text { sinon. }
\end{array}
$$

On observe sans difficulté que $\tilde{\varphi}_{1}(L)(v) \geqslant \tilde{\varphi}_{2}(L)(v)$ pour tout $v \in \frac{1}{b} \mathbb{Z}$; ainsi $\varphi_{1}(L)=\tilde{\varphi}_{1}(L)$ et $\varphi_{2}(L)=$ $\tilde{\varphi}_{2}(L)$. Il est maintenant facile de vérifier la proposition 1.2 sur cet exemple et en particulier de décrire les fonctions $\psi_{1}(L)$ et $\psi_{2}(L)$. On trouve :

$$
\begin{array}{rlrl}
\psi_{1}(L): \mu & \mapsto-\infty & & \operatorname{si} \mu<b(\alpha+\delta-\gamma)-\delta \\
\mu & \mapsto b^{-1}(\mu+\gamma) & & \text { sinon } \\
\psi_{2}(L): \mu & \mapsto-\infty & & \text { si } \mu<b \gamma-\alpha \\
\mu & \mapsto b^{-1}(\mu+\alpha) & & \text { si } b \gamma-\alpha \leqslant \mu<(b-1)(\alpha+\delta-\gamma) \\
\mu & \mapsto b^{-1}(\mu+\alpha+\delta-\gamma) & \text { sinon. }
\end{array}
$$

Les entiers $\mu_{1}(L)$ et $\mu_{2}(L)$ valent donc respectivement $b(\alpha+\delta-\gamma)-\delta$ et $b \gamma-\alpha$, et l'on peut vérifier par un calcul indépendant que ce sont bien les exposants des diviseurs élémentaires du $k[[u]]$-module engendré $\operatorname{par} \sigma(L)$ par rapport à $L$.

\subsubsection{Prolongement des fonctions $\varphi_{i}$}

Pour la suite, il sera commode, afin de mieux visualiser les fonctions $\varphi_{i}(L)$ de les prolonger à tout $\mathbb{R}$ en posant

$$
\varphi_{i}(q)=\varphi_{i}(v)+b(q-v) \quad \text { pour tout } q \in\left[v, v+\frac{1}{b}[\right.
$$

avec la convention que $-\infty+x=-\infty$ pour tout nombre réel $x$. On prolonge de la même façon les fonctions $\tilde{\varphi}_{i}(L)$. Il est alors clair que pour tout réel $q$ les nombres $\varphi_{1}(L)(q), \ldots, \varphi_{d}(L)(q)$ sont les mêmes que $\tilde{\varphi}_{1}(L)(q), \ldots, \tilde{\varphi}_{d}(L)(q)$ mais triés par ordre décroissant. Il est également évident que les fonctions $\tilde{\varphi}_{i}(L)$ et $\varphi_{i}(L)$ sont continues à droite, affines par morceaux, et que leurs dérivées valent $b$ partout où elles sont définies. Les fonctions $\psi_{j}(L)$ données par la proposition 1.2 se prolongent elles aussi à $\mathbb{R}$ tout entier en convenant qu'elles valent $-\infty$ sur l'intervalle ] $-\infty, \mu_{j}(L)$ [ et qu'elles sont affines de pente $\frac{1}{b}$ sur tout intervalle de la forme $\left[\mu, \mu+1\right.$ [ où $\mu$ est un nombre entier supérieur ou égal à $\mu_{j}(L)$. Ces fonctions ainsi prolongées vérifient encore la condition de l'alinéa 3 de la proposition 1.2 lorsque $v$ et $\mu$ sont des éléments de $\mathbb{R}$.

La méthode que l'on a employée pour prolonger les fonctions $\varphi_{i}$ et $\psi_{j}$ à tout $\mathbb{R}$ peut sembler artificielle, mais en fait il n'en est rien comme le montre la proposition suivante.

Proposition 1.4. On note $k\left[\left[u^{1 / \infty}\right]\right]=\bigcup_{n \geqslant 1} k\left[\left[u^{1 / n}\right]\right]$ (resp. $\left.k\left(\left(u^{1 / \infty}\right)\right)=\bigcup_{n \geqslant 1} k\left(\left(u^{1 / n}\right)\right)\right)$ l'anneau (resp. le corps) des séries de Puiseux à coefficients dans $k$. Alors pour tout $i \in\{1, \ldots, d\}$ et tout nombre rationnel $q$, on $a$ :

$$
\tilde{\varphi}_{i}(L)(q)=\sup _{\substack{x \in k\left[\left[u^{1 / \infty}\right]\right] \otimes_{k[[u]]} L \\ \operatorname{val}_{M, \mathbb{Q}}(x)=(q, i)}}\left(\sup \left\{n \in \mathbb{Q} \mid \sigma(x) \in u^{n}\left(k\left[\left[u^{1 / \infty}\right]\right] \otimes_{k[[u]]} L\right)\right\}\right)
$$

où $\operatorname{val}_{M, \mathbb{Q}}$ désigne le prolongement naturel de $\operatorname{val}_{M}$ à $k\left(\left(u^{1 / \infty}\right)\right) \otimes_{k((u))} M$.

Remarque 1.5. En d'autres termes, la proposition dit que la fonction

$$
q \mapsto \sup _{\substack{x \in k\left[\left[u^{1 / \infty}\right]\right] \otimes_{k[[u]]} L \\ \operatorname{val}_{M, \mathbb{Q}}(x)=(q, i)}}\left(\sup \left\{n \in \mathbb{Q} \mid \sigma(x) \in u^{n}\left(k\left[\left[u^{1 / \infty}\right]\right] \otimes_{k[[u]]} L\right)\right\}\right)
$$

est entièrement déterminée par ses valeurs sur l'ensemble $\frac{1}{b} \mathbb{Z}$ et que, de surcrôit, pour calculer les valeurs en ces points, on peut se contenter d'étendre les scalaires à $k\left(\left(u^{1 / b}\right)\right)$ (sans aller, donc, jusqu'aux séries de Puiseux). 
Démonstration. Étant donné que la proposition ne sera pas utilisée dans la suite, on se contente de donner quelques indications sur sa preuve. L'idée directrice est de comprendre comment l'on peut calculer de façon algorithmique la borne supérieure qui apparaît dans l'énoncé de la proposition, notée $f_{i}(L)(q)$ dans le restant de la preuve. Dans la suite, on notera également $\left(e_{1}, \ldots, e_{d}\right)$ la base canonique de $M$. L'entier $i$ restant fixé, on explique tout d'abord comment calculer la borne inférieure des $q$ tels qu'il existe dans $k\left[\left[u^{1 / \infty}\right]\right] \otimes_{k[[u]]} L$ des éléments de valuation $(q, i)$. Soit $q_{i}$ cette borne inférieure. Dire que $k\left[\left[u^{1 / \infty}\right]\right] \otimes_{k[[u]]} L$ contient un élément de valuation $(q, i)$ signifie exactement qu'il existe un nombre rationnel $\varepsilon>0$ tel que

$$
u^{q} e_{i} \in L+\sum_{j=1}^{i} u^{q+\varepsilon} e_{j} k\left[\left[u^{1 / \infty}\right]\right]+\sum_{j=i+1}^{d} u^{q} e_{j} k\left[\left[u^{1 / \infty}\right]\right] .
$$

Si l'on fixe $m_{1}, \ldots, m_{d}$ une base de $L$ et que l'on note $M_{i}$ (resp. $E_{i}$ ) le vecteur colonne des coordonnées de $m_{i}$ (resp. $e_{i}$ ) dans la base canonique, la condition précédente signifie que le vecteur colonne $u^{q} E_{i}$ est dans l'image de la matrice par blocs

$$
\left(M_{1}\left|M_{2}\right| \cdots\left|M_{d}\right| u^{q+\varepsilon} E_{1}|\cdots| u^{q+\varepsilon} E_{i}\left|u^{q} E_{i+1}\right| \cdots \mid u^{q} E_{d}\right)
$$

c'est-à-dire dans le module engendré par les vecteurs colonne de cette matrice. On peut à présent effectuer des opérations sur les colonnes de la matrice précédente (ce qui ne modifie pas son image) pour se ramener à permutation des lignes près à une matrice de la forme

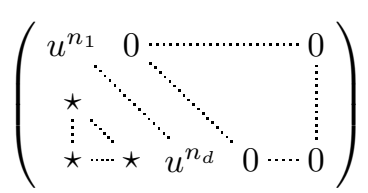

pour certains nombres rationnels $n_{j}(1 \leqslant j \leqslant d)$ rangés par ordre croissant. En outre, un examen de l'algorithme classique de calcul de la forme précédente permet d'établir la dépendance en $q$ et $\varepsilon$ (pourvu que ce dernier reste suffisamment petit) des $n_{j}$ : on trouve qu'il existe des entiers $v_{1}<\cdots<v_{n}$ et $c_{0, j}<\cdots<c_{n, j}$ tels que, en posant $v_{0}=-\infty$ et $v_{n+1}=+\infty$, on ait pour tout $j$, et sur chaque intervalle $\left[v_{s}, v_{s+1}\left[\right.\right.$, soit $n_{j}=c_{s, j}$, soit $n_{j}=q+c_{s, j}$, soit $n_{j}=q+\varepsilon+c_{s, j}$. Il est enfin possible d'exprimer $q_{i}$ en fonction des $c_{s, j}$, à partir de quoi l'on déduit que $q_{i}$ est entier. Il résulte également de ces considérations qu'il existe dans $L$ (sans tensoriser par $k\left[\left[u^{1 / \infty}\right]\right]$ ) un élément de valuation $\left(q_{i}, i\right)$ puisque dans le cas où $q$ est un nombre entier, toutes les opérations effectuées peuvent se faire dans $k[[u]]$.

Pour $q<q_{i}$, la proposition est clairement vraie puisque les deux nombres qui apparaissent dans l'égalité à établir valent tous deux $-\infty$. D'autre part, dans tous les cas, le nombre $f_{i}(L)(q)$ s'interprète aussi comme la borne inférieure des nombres rationnels $n$ tels que l'implication suivante soit vraie :

$$
\left(x \in k\left[\left[u^{1 / \infty}\right]\right] \otimes_{k[[u]]} L \text { et } \operatorname{val}(x)=(q, i)\right) \quad \Longrightarrow \quad \sigma(x) \notin u^{n}\left(k\left[\left[u^{1 / \infty}\right]\right] \otimes_{k[[u]]} L\right) .
$$

Or, si $z_{i} \in L$ est un élément fixé de valuation $\left(q_{i}, i\right)$ et si l'on suppose $q \geqslant q_{i}$, un élément $x$ vérifie la prémisse de l'implication si, et seulement si

$$
x-u^{q-q_{i}} z_{i} \in\left(k\left[\left[u^{1 / \infty}\right]\right] \otimes_{k[[u]]} L\right) \cap\left(\sum_{j=1}^{i} u^{q+\varepsilon} e_{j}+\sum_{j=i+1}^{d} u^{q} e_{j}\right)
$$

pour un certain $\varepsilon>0$. On conclut alors de manière semblable à ce qui a été déjà fait : on commence par calculer l'intersection qui apparaît dans la formule précédente en effectuant des opérations sur les lignes d'une matrice, et réinjectant cela dans l'implication (1.3), on trouve qu'il existe des entiers $v_{1}<\cdots<v_{n}$ et $c_{0}<\cdots<c_{n}$ tel que sur chaque intervalle $\left[\frac{v_{s}}{b}, \frac{v_{s}+1}{b}\left[\right.\right.$, on ait $f_{i}(L)(q)=b q-c_{s}$. Ce faisant, on obtient également que $f_{i}(L)(q)=\tilde{\varphi}_{i}(L)(q)$ si $q \in \frac{1}{b} \mathbb{Z}$, d'où il résulte la proposition.

En s'autorisant à travailler dans des corps encore plus gros que $k\left(\left(u^{1 / \infty}\right)\right)$, on peut aussi interpréter les nombres $\tilde{\varphi}_{i}(L)(q)$ pour $q \in \mathbb{R}$ comme des bornes supérieures du type précédent. Par exemple, on peut considérer l'anneau $k\left[\left[u^{\mathbb{R}^{+}}\right]\right]$formé des séries formelles $\sum_{i \in I} a_{i} u^{i}$ où $I \subset \mathbb{R}^{+}$est un monoïde de type fini. 
Celui-ci est encore muni d'une valuation naturelle qui permet de définir val $M, \mathbb{R}$ sur $k\left[\left[u^{\mathbb{R}^{+}}\right]\right] \otimes_{k[[u]]} M$. On a alors pour tout nombre réel $q$ :

$$
\tilde{\varphi}_{i}(L)(q)=\sup _{\substack{x \in k\left[\left[u^{\mathbb{R}^{+}}\right]\right] \otimes_{k[[u]]} L \\ \operatorname{val}_{M, \mathbb{R}}(x)=(q, i)}}\left(\sup \left\{n \in \mathbb{R} \mid \sigma(x) \in u^{n}\left(k\left[\left[u^{\mathbb{R}^{+}}\right]\right] \otimes_{k[[u]]} L\right)\right\}\right)
$$

la démonstration étant en tout point analogue à celle de la proposition précédente.

\subsection{Paramétrisation de l'espace des fonctions $\varphi$}

L'objectif de ce numéro est de décrire complètement les $d$-uplets $\varphi=\left(\varphi_{1}, \ldots, \varphi_{d}\right)$ où les $\varphi_{i}$ sont des fonctions de $\mathbb{R}$ dans $\mathbb{R} \cup\{-\infty\}$ qui satisfont aux conditions suivantes :

1. on a $\varphi_{1} \geqslant \varphi_{2} \geqslant \cdots \geqslant \varphi_{d}$

2. les fonctions $\varphi_{i}$ sont strictement croissantes fet continues à droite ;

3. pour tout $i$, il existe un nombre réel $q_{i}$ (nécessairement unique) tel que

- la fonction $\varphi_{i}$ prend des valeurs finies exactement sur l'intervalle $\left[q_{i},+\infty[\right.$,

- la fonction $\varphi_{i}$ est affine par morceaux sur $\left[q_{i},+\infty\right.$ [ et pour presque tout $q$ dans cet intervalle, on a $\varphi_{i}^{\prime}(q)=b$, et

- pour $q$ suffisamment grand, on a $\varphi_{i}(q)=b q-q_{i}$;

4. pour $j \in\{1, \ldots, d\}$, il existe des fonctions strictement croissantes et continues à droites $\psi_{j}: \mathbb{R} \rightarrow$ $\mathbb{R} \cup\{-\infty\}$ telles que $\psi_{1} \leqslant \psi_{2} \leqslant \cdots \leqslant \psi_{d}$ et pour tout couple $(q, \mu) \in \mathbb{R}^{2}$, il y a autant d'indices $i \in\{1, \ldots, d\}$ tels que $\mu=\varphi_{i}(q)$ que d'indices $j \in\{1, \ldots, d\}$ tels que $q=\psi_{j}(\mu)$.

À partir de maintenant, on note $\Phi$ l'ensemble des $d$-uplets de fonctions $\varphi=\left(\varphi_{1}, \cdots, \varphi_{d}\right)$ vérifiant les conditions précédentes. La proposition 1.2, dit que les $\varphi(L)=\left(\varphi_{1}(L), \ldots, \varphi_{d}(L)\right)$ provenant d'un réseau $L \subset M$ définissent des éléments de $\Phi$. Par contre, il n'est pas vrai que, réciproquement, tout élément de $\Phi$ s'obtient de cette manière. En effet, si $\varphi$ provient d'un réseau, il vérifie en outre au moins les deux propriétés supplémentaires suivantes :

5. pour tout $i$, le réel $q_{i}$ est un nombre entier;

6. pour tout $i$, les réels en lesquels $\varphi_{i}$ est discontinue appartiennent à $\frac{1}{b} \mathbb{Z}$.

Ces deux dernièrs conditions seront appelées conditions d'intégrité dans la suite de cet article, tandis que l'ensemble des éléments de $\Phi$ qui les satisfont sera noté $\Phi_{\mathbb{Z}}$.

\subsubsection{Les réels $q_{i, j}$ et $\mu_{i, j}$}

Soient $\varphi=\left(\varphi_{1}, \ldots, \varphi_{d}\right) \in \Phi$ et $\psi=\left(\psi_{1}, \ldots, \psi_{d}\right)$ le $d$-uplet de fonctions correspondant.

On suppose pour commencer - et il s'agit d'une hypothèse qui évitera bien des problèmes techniques - que $\varphi_{1}>\cdots>\varphi_{d}$ où, étant donné deux fonctions $f, g: \mathbb{R} \rightarrow \mathbb{R} \cup\{-\infty\}$, on convient que $f>g$ si $f(q) \geqslant g(q)$ pour tout réel $q$ et que l'inégalité est stricte dès que $f(q) \neq-\infty$. On prolonge les fonctions $\varphi_{i}$ et $\psi_{j}$ à $\mathbb{R} \cup\{-\infty\}$ en posant $\varphi_{i}(-\infty)=\psi_{j}(-\infty)=-\infty$. Pour tout couple $(i, j)$ d'entiers vérifiant $1 \leqslant i \leqslant j \leqslant d$, on définit :

- le nombre $q_{i, j}$ comme la borne inférieure des nombres réels $q$ tels que $\psi_{j} \circ \varphi_{i}(q) \geqslant q$;

- le nombre $\mu_{i, j}$ comme la borne inférieure des nombres réels $\mu$ tels que $\varphi_{i} \circ \psi_{j}(\mu) \geqslant \mu$.

L'ordre dans lequel sont classés les $\varphi_{i}$ et les $\psi_{j}$ impose que les fonctions $\varphi_{i}$ et $\psi_{i}$ sont inverses l'une de l'autre sur des voisinages de $+\infty$; il s'ensuit qu'il existe toujours des $q$ et des $\mu$ satisfaisant les inégalités précédentes. Par ailleurs si $q$ (resp. $\mu$ ) est suffisamment petit, on a $\varphi_{i}(q)=-\infty$ pour tout $i$ (resp. $\psi_{j}(\mu)=$ $-\infty$ pour tout $j$ ). On en déduit que les $q_{i, j}$ et $\mu_{i, j}$ sont bien des nombres réels. Enfin, il est clair que $q_{i, j} \leqslant q_{i+1, j}, q_{i, j} \geqslant q_{i, j+1}, \mu_{i, j} \leqslant \mu_{i+1, j}$ et $\mu_{i, j} \geqslant \mu_{i, j+1}$ pour tout couple $(i, j)$ pour lequel cela a un sens. Dans la suite, pour des raisons pratiques, on posera également $q_{i, i-1}=\mu_{j+1, j}=+\infty$ pour tous indices $i$ et $j$.

4. On rappelle que l'on entend par là que les $\varphi_{i}$ sont croissantes et strictement croissantes sur l'intervalle où elles prennent des valeurs finies 


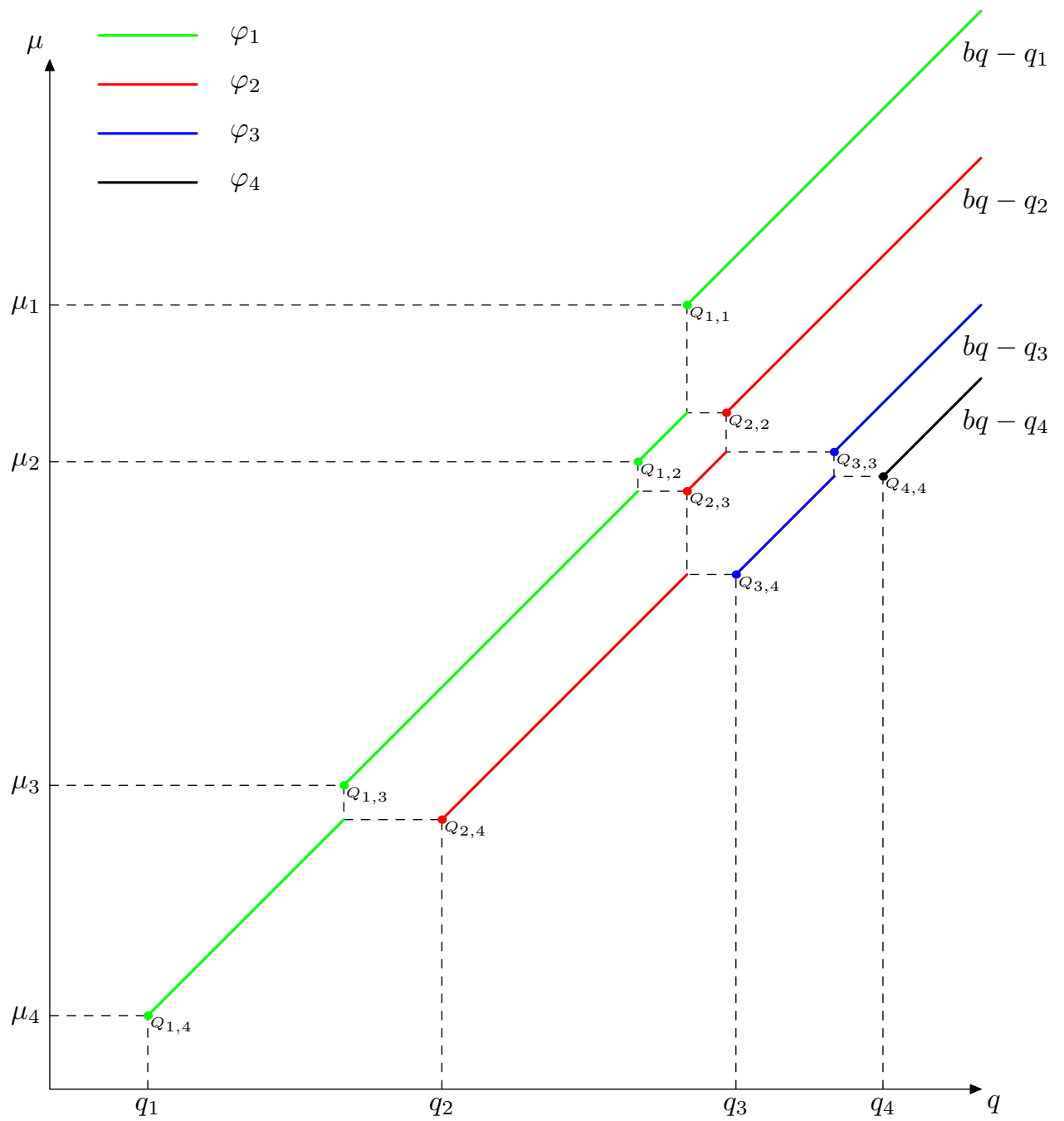

FIGURE 1 - Un exemple de $d$-uplet $\left(\varphi_{1}, \ldots, \varphi_{d}\right)$ appartenant à $\Phi$ avec $d=4$

Les points $Q_{i, j}$ qui apparaissent sur le graphique sont ceux de coordonnées $\left(q_{i, j}, \mu_{i, j}\right)$.

Les valeurs $q_{i}=q_{i, d}$ indiquées sur l'axe des abscisses correspondent aux endroits à partir desquels les fonctions $\varphi_{i}$ prennent des valeurs finies.

Les valeurs $\mu_{j}=\mu_{1, j}$ indiquées sur l'axe des ordonnées correspondent, quant à elles, aux endroits à partir desquels les fonctions $\psi_{j}$ prennent des valeurs finies et aussi, dans le cas où le $d$-uplet $\left(\varphi_{1}, \ldots, \varphi_{d}\right)$ provient d'un réseau $L$, aux exposants des diviseurs élémentaires de $\sigma\left(k\left[\left[u^{1 / b}\right]\right] \otimes_{k[[u]]} L\right)$ par rapport à $L$. 
Lemme 1.6. Pour tout entiers $i$ et $j$ tels que $1 \leqslant i \leqslant j \leqslant d$, on a

$$
\mu_{i, j} \leqslant \varphi_{i}\left(q_{i, j}\right) \quad \text { et } \quad q_{i, j} \leqslant \psi_{j}\left(\mu_{i, j}\right)
$$

De plus ces inégalités sont des égalités si, et seulement s'il existe q tel que $\psi_{j} \circ \varphi_{i}(q)=q$ si, et seulement s'il existe $\mu$ tel que $\varphi_{i} \circ \psi_{j}(\mu)=\mu$.

Démonstration. La croissance et la continuité à droite des fonctions $\varphi_{i}$ et $\psi_{j}$ impliquent que $\psi_{j} \circ \varphi_{i}$ est aussi continue à droite. On en déduit que $\psi_{j} \circ \varphi_{i}\left(q_{i, j}\right) \geqslant q_{i, j}$. À partir de là, en appliquant $\varphi_{i}$, on obtient $\varphi_{i} \circ \psi_{j}(\mu) \geqslant \mu$ avec $\mu=\varphi_{i}\left(q_{i, j}\right)$. Par définition de la borne inférieure, il vient $\mu_{i, j} \leqslant \mu$ comme annoncé. On démontre de même l'autre inégalité.

Il est clair que si les inégalités sont des égalités, il existe $q$ et $\mu$ satisfaisant à la condition du lemme : il suffit de prendre $q=q_{i, j}$ et $\mu=\mu_{i, j}$. On suppose maintenant qu'il existe $q$ tel que $\psi_{j} \circ \varphi_{i}(q)=q$. Du fait que la fonction $\psi_{j} \circ \varphi_{i}$ - id est en escalier (puisqu'elle est affine par morceaux et que sa dérivée s'annule partout où elle est définie), on déduit facilement que $\psi_{j} \circ \varphi_{i}\left(q_{i, j}\right)=q_{i, j}$. En appliquant maintenant $\psi_{j}$ à la première égalité du lemme, on trouve que $\psi_{j}\left(\mu_{i, j}\right) \leqslant q_{i, j}$ et donc finalement que $\psi_{j}\left(\mu_{i, j}\right)=q_{i, j}$. L'autre égalité se démontre de même analogue en appliquant $\varphi_{i}$ à la deuxième inégalité du lemme. Le cas où il existe $\mu$ tel que $\varphi_{i} \circ \psi_{j}(\mu)=\mu$ se traite pareillement.

Dans le cas où l'une des inégalités du lemme est stricte, on dira que le couple $(i, j)$ est dégénéré. Il est à noter que les couples de la forme $(i, i)$ ne sont jamais dégénérés puisque l'on a dit que sur des voisinages de l'infini les fonctions $\varphi_{i}$ et $\psi_{i}$ étaient inverses l'une de l'autre. En particulier, on a toujours $\varphi_{i}\left(q_{i, i}\right)=\mu_{i, i}$ et $\psi_{i}\left(\mu_{i, i}\right)=q_{i, i}$.

Proposition 1.7. Soit $\varphi=\left(\varphi_{1}, \ldots, \varphi_{d}\right) \in \Phi$ tel que $\varphi_{1}>\cdots>\varphi_{d}$. Alors, pour tout couple $(i, j)$ avec $1 \leqslant i \leqslant j \leqslant d$, les fonctions $\varphi_{i}$ et $\psi_{j}$ définissent par restriction des bijections

$$
\varphi_{i \mid\left[q_{i, j}, q_{i, j-1}[\right.}:\left[q_{i, j}, q_{i, j-1}\left[\rightarrow \left[\mu_{i, j}, \mu_{i+1, j}\left[\quad \text { et } \quad \psi_{j \mid\left[\mu_{i, j}, \mu_{i+1, j}[\right.}:\left[\mu_{i, j}, \mu_{i+1, j}\left[\rightarrow \left[q_{i, j}, q_{i, j-1}[\right.\right.\right.\right.\right.\right.\right.
$$

inverses l'une de l'autre. (On notera qu'il est possible que les intervalles précédents soient vides.)

De plus, pour tout $i$ (resp. tout $j$ ), la fonction $\varphi_{i}\left(\right.$ resp. $\left.\psi_{j}\right)$ vaut $-\infty$ sur l'intervalle $]-\infty, q_{i, d}[$ (resp. l'intervalle ] $-\infty, \mu_{1, j}[$ ).

Remarque 1.8. Telle quelle, la proposition est fausse sans l'hypothèse $\varphi_{1}>\cdots>\varphi_{d}$. On expliquera rapidement au $\$ 1.2 .3$ comment modifier la définition des $q_{i, j}$ et $\mu_{i, j}$ pour que la proposition s'étende sans cette hypothèse.

Démonstration. On raisonne par récurrence sur $j$ en commençant par traiter le cas $j=1$. Puisque $\psi_{1}$ est strictement croissante, il est clair qu'elle prend des valeurs finies exactement sur un intervalle de la forme $\left[\mu_{1},+\infty\left[\right.\right.$ pour $\mu_{1} \in \mathbb{R}$. Du fait que $\psi_{1}$ est la plus petite des fonctions $\psi_{j}$, on déduit que la réunion des graphes des $\varphi_{i}$ n'intersecte pas la région du plan suivante :

$$
D_{1}=\left\{(q, \mu) \in \mathbb{R}^{2} \mid q<\psi_{1}(\mu)\right\} .
$$

Soit $\mu$ un réel plus grand ou égal à $\mu_{1}$. On pose $q=\psi_{1}(\mu)$ et et on considère un réel $\mu^{\prime}$ strictement supérieur à $\mu$. Étant donné que $\psi_{1}$ est croissante, le couple $\left(q, \mu^{\prime}\right)$ est dans $D_{1}$, ce qui signifie que $\varphi_{i}(q) \neq \mu^{\prime}$ pour tout $i$. Comme ceci est vrai pour tout $\mu^{\prime}>\mu$, on obtient $\varphi_{i}(q) \leqslant \mu$, i.e $\varphi_{i} \circ \psi_{1}(\mu) \leqslant \mu$. Par ailleurs, on sait que cette inégalité doit être une égalité pour au moins un $i$. Si $i_{0}$ est cet indice privilégié, on a $\mu=\varphi_{i_{0}} \circ \psi_{1}(\mu) \leqslant \varphi_{1} \circ \psi_{1}(\mu) \leqslant \mu$ car les $\varphi_{i}$ sont triés par ordre décroissant. Ainsi $\varphi_{1} \circ \psi_{1}(\mu)=\mu$, et ce pour tout $\mu \geqslant \mu_{1}$. On en déduit que $\mu_{1,1} \leqslant \mu_{1}$. Mais comme $\psi_{1}$ vaut $-\infty$ sur les $\mu<\mu_{1}$, il vient $\mu_{1,1}=\mu_{1}$ et donc $q_{1,1}=\psi_{1}\left(\mu_{1}\right)$ par le lemme 1.6. En outre, sur l'intervalle $\left[\mu_{1,1},+\infty\left[\right.\right.$ la fonction $\psi_{1}$ est inversible à gauche, et son inverse à gauche est la restriction de $\varphi_{1}$ à $\left[q_{1,1},+\infty[\right.$. Du fait que ces fonctions sont en outre affines par morceaux et strictement croissantes, on en déduit facilement ce qui est énoncé dans la proposition.

Plutôt que de traiter l'hérédité de la récurrence dans le cas général — ce qui multiplierait encore les notations - on se contente d'expliquer comment le cas $\ll j=2 \gg$ se déduit de ce que l'on vient de faire, les arguments pour les $j$ supérieurs étant similaires. Comme précédemment, on commence par remarquer que la fonction $\psi_{2}$ prend des valeurs finies exactement sur un intervalle de la forme $\left[\mu_{2},+\infty\right.$ [ pour un certain nombre réel $\mu_{2}$. De $\psi_{2}\left(\mu_{1,1}\right) \geqslant \psi_{1}\left(\mu_{1,1}\right)>-\infty$, on tire $\mu_{2} \leqslant \mu_{1,1}$. On introduit le domaine :

$$
D_{2}=\left\{(q, \mu) \in \mathbb{R}^{2} \mid q<\psi_{2}(\mu)\right\} .
$$


Des résultats de l'étude menée pour $j=1$, on déduit que $D_{2}$ intersecte la réunion des graphes de $\varphi_{i}$ au plus selon le graphe de la restriction de $\varphi_{1}$ à l'intervalle $\left[q_{1,1},+\infty\right.$. Soient $\mu \geqslant \mu_{2}$ et $q=\psi_{2}(\mu)$. On considère un nombre réel $\mu^{\prime}>\mu$. Le couple $\left(q, \mu^{\prime}\right)$ appartenant à $D_{2}$, il en résulte que :

- si $q<q_{1,1}$, alors $\varphi_{i}(q) \neq \mu^{\prime}$ pour tout $i \in\{1, \ldots, d\}$;

- si $q \geqslant q_{1,1}$, alors $\varphi_{i}(q) \neq \mu^{\prime}$ pour tout $i \in\{2, \ldots, d\}$.

Les conclusions précédentes étant valables pour tout $\mu^{\prime}>\mu$, on peut remplacer dans leurs énoncés $\ll \varphi_{i}(q) \neq$ $\mu^{\prime} \gg \operatorname{par} \ll \varphi_{i}(q) \leqslant \mu \gg$. En utilisant le fait que les fonctions $\varphi_{i}$ sont triées par ordre décroissant, et que la fonction $\varphi_{1}$ est connue sur $\left[q_{1,1},+\infty\left[\right.\right.$, on obtient pour tout $\mu \geqslant \mu_{2}$ :

- si $\psi_{2}(\mu)<q_{1,1}$, alors $\varphi_{1} \circ \psi_{2}(\mu)=\mu$;

- si $\psi_{2}(\mu) \geqslant q_{1,1}$, alors $\varphi_{2} \circ \psi_{2}(\mu)=\mu$, et donc $\varphi_{1} \circ \psi_{2}(\mu) \geqslant \mu$.

On déduit déjà de cela que $\mu_{1,2}=\mu_{2}$ puisque $\varphi_{1} \circ \psi_{2}(\mu)$ vaut $-\infty$ si $\mu<\mu_{2}$ et est supérieur ou égal à $\mu$ sinon. On a également $\psi_{2}\left(\mu_{2,2}\right)=q_{2,2}$ et $\varphi_{2}\left(q_{2,2}\right)=\mu_{2,2}$ étant donné que le couple $(2,2)$ n'est pas dégénéré. Soit $\mu^{\prime}$ l'infimum des nombres réels $\mu$ tels que $\psi_{2}(\mu)>q_{1,1}$. La restriction de $\psi_{2}$ à $\left[\mu_{1,2}, \mu^{\prime}[\right.$ admet alors pour inverse $\varphi_{1}$ tandis que sa restriction à $\left[\mu^{\prime},+\infty\left[\right.\right.$ admet pour inverse $\varphi_{2}$. Pour conclure, il suffit donc de montrer que $\mu^{\prime}=\mu_{2,2}$ et que si $\mu_{1,2}<\mu_{2,2}$ alors $\psi_{2}\left(\mu_{1,2}\right)=q_{1,2}$. Du fait que $\varphi_{2} \circ \psi_{2}\left(\mu^{\prime}\right)=$ $\mu^{\prime}$, on déduit que $\mu_{2,2} \leqslant \mu^{\prime}$. Mais si l'inégalité était stricte, on aurait $\varphi_{1}=\varphi_{2}$ sur l'intervalle de la forme $\left[q_{1,1}-\varepsilon, q_{1,1}[\right.$ (pour $\varepsilon>0$ ), ce que l'on a exclu au départ. L'autre point résulte maintenant des descriptions que l'on vient d'obtenir.

Étant donné qu'une fonction définie sur un intervalle à valeurs dans un autre intervalle, qui est à la fois affine par morceaux et bijective est affine, on déduit facilement de la proposition la description suivante des fonctions $\varphi_{i}$ et $\psi_{j}$ :

$$
\begin{array}{rlll}
\varphi_{i}: q & \mapsto-\infty & \text { si } q<q_{i, d} \\
q & \mapsto b\left(q-q_{i, j}\right)+\mu_{i, j} \quad & \text { si } q_{i, j} \leqslant q<q_{i, j-1}, \quad \text { et ce pour tout } j \in\{i, \ldots, d\} \\
\psi_{j}: \quad \mu & \mapsto-\infty & \text { si } \mu<\mu_{1, j} \\
\mu & \mapsto b^{-1}\left(\mu-\mu_{i, j}\right)+q_{i, j} & \text { si } \mu_{i, j} \leqslant \mu<\mu_{i+1, j}, \quad \text { et ce pour tout } i \in\{1, \ldots, j\}
\end{array}
$$

Il résulte en particulier de cette écriture que les fonctions $\varphi_{i}$ sont entièrement déterminées par la donnée des $q_{i, j}$ et $\mu_{i, j}$.

\subsubsection{Relations entre les $q_{i, j}$ et $\mu_{i, j}$}

On sait déjà que les nombres $q_{i, j}$ et $\mu_{i, j}$ ne peuvent être quelconques puisque ceux-ci vérifient les inégalités $q_{i, j} \leqslant q_{i+1, j}, q_{i, j} \geqslant q_{i, j+1}, \mu_{i, j} \leqslant \mu_{i+1, j}$ et $\mu_{i, j} \geqslant \mu_{i, j+1}$. Dans ce paragraphe, on détermine un certain nombres d'autres contraintes auxquelles ils doivent satisfaire.

Relations égalitaires On considère $\varphi=\left(\varphi_{1}, \ldots, \varphi_{d}\right) \in \Phi$ et on suppose encore $\varphi_{1}>\cdots>\varphi_{d}$. Par définition, au voisinage de $+\infty$, la fonction $\varphi_{i}$ est donnée par $q \mapsto b q-q_{i, d}$ (puisque la fonction $\varphi_{i}$ prend des valeurs finis à partir de $q_{i, d}$ ). En comparant avec la forme de $\varphi_{i}$ obtenu ci-dessus, on trouve :

$$
\mu_{i, i}=b q_{i, i}-q_{i, d}
$$

pour tout $i \in\{1, \ldots, d\}$. D'autre part, par la proposition 1.7, la fonction $\varphi_{i}$ réalise une bijection de l'intervalle $\left[q_{i, j}, q_{i, j-1}\left[\right.\right.$ sur l'intervalle $\left[\mu_{i, j}, \mu_{i+1, j}[\right.$. Comme on sait que cette fonction dilate la mesure de Lebesgue d'un facteur $b$, il vient :

$$
\mu_{i+1, j}-\mu_{i, j}=b\left(q_{i, j-1}-q_{i, j}\right)
$$

pour tout $i, j \in\{1, \ldots, d\}$ tels que $1 \leqslant i<j \leqslant d$. À partir des ces deux relations, on voit facilement que les $\mu_{i, j}$ s'expriment en fonction des $q_{i, j}$ :

$$
\mu_{i, j}=b q_{j, j}-q_{j, d}+b \cdot \sum_{s=i}^{j-1}\left(q_{s, j}-q_{s, j-1}\right)
$$


pour tout couple $(i, j)$ tel que $1 \leqslant i \leqslant j \leqslant d$. En fait, la formule $(1.7)$ implique réciproquement les formules (1.5) et (1.6). Ainsi, on peut décider d'oublier ces deux dernières relations et de ne travailler qu'avec les $q_{i, j}$. On peut également inverser les formules (1.7) et exprimer les $q_{i, j}$ en fonction des $\mu_{i, j}$; on obtient pour $1 \leqslant i \leqslant j \leqslant d$

$$
q_{i, j}=\frac{1}{b-1} \cdot\left(\mu_{i, i}+\sum_{s=i+1}^{j}\left(\mu_{i, s}-\mu_{i+1, s}\right)+\sum_{s=j+1}^{d} \frac{\mu_{i, s}-\mu_{i+1, s}}{b}\right) .
$$

Il est donc également possible d'oublier les $q_{i, j}$ et de travailler uniquement avec les $\mu_{i, j}$. L'avantage, néanmoins, de continuer à considérer simultanément les $q_{i, j}$ et les $\mu_{i, j}$ réside dans le fait que les formules qui apparaîtront dans la suite pourront souvent s'écrire manière plus agréable.

Relations inégalitaires On rappelle que l'on a déjà vu les inégalités

$$
\begin{aligned}
& q_{i, j} \geqslant q_{i, j+1} \quad ; \quad \mu_{i, j} \leqslant \mu_{i+1, j} \\
& q_{i, j} \leqslant q_{i+1, j} \quad ; \quad \mu_{i, j} \geqslant \mu_{i, j+1}
\end{aligned}
$$

pour tout couple $(i, j)$ pour lequel cela a un sens.

Lemme 1.9. Avec les notations précédentes, on a les relations supplémentaires :

$$
q_{i, j} \leqslant q_{i+1, j+1} \quad \text { et } \quad \mu_{i, j} \geqslant \mu_{i+1, j+1}
$$

pour tout couple $(i, j)$ tel que $1 \leqslant i<j \leqslant d$.

Démonstration. On démontre seulement la première inégalité, la seconde étant complètement analogue. Si $q_{i, j}=q_{i, j+1}$ ou $q_{i+1, j+1}=q_{i+1, j}$, l'inégalité résulte de $(1.10)$. On peut donc supposer que $q_{i, j}>q_{i, j+1}$ et $q_{i+1, j+1}<q_{i+1, j}$. Dans ce cas, on applique la proposition 1.7 qui nous assure que la fonction $\psi_{j+1}$ réalise une bijection croissante de $\left[\mu_{i, j+1}, \mu_{i+1, j+1}\left[\right.\right.$ dans $\left[q_{i, j+1}, q_{i, j}\left[\right.\right.$, et également de $\left[\mu_{i+1, j+1}, \mu_{i+2, j+1}[\right.$ dans $\left[q_{i+1, j+1}, q_{i+1, j}\left[\right.\right.$. Par hypothèse, tous ces intervalles sont non vides. On en déduit que $\lim _{\mu \rightarrow \mu_{i+1, j+1}^{-}} \psi_{j+1}(\mu)=$ $q_{i, j}$ et que $\psi_{j+1}\left(\mu_{i+1, j+1}\right)=q_{i+1, j+1}$. En utilisant la croissance de $\psi_{j+1}$, on obtient finalement $q_{i, j} \leqslant$ $q_{i+1, j+1}$ comme voulu.

En fait, les inégalités (1.10) résultent de (1.9) et (1.11). En outre, en vertu de l'égalité (1.6), les deux inégalités de la ligne (1.9) se déduisent mutuellement l'une de l'autre. Ainsi, les six jeux d'inégalités obtenues précédemment se résument finalement aux trois suivants :

$$
q_{i, j} \geqslant q_{i, j+1} \quad ; \quad q_{i, j} \leqslant q_{i+1, j+1} \quad ; \quad \mu_{i, j} \geqslant \mu_{i+1, j+1} .
$$

où $(i, j)$ parcourt l'ensemble des couples d'entiers tels que $1 \leqslant i \leqslant j<d$. Bien entendu, par ailleurs, les inégalités portant sur les $\mu_{i, j}$ peuvent se réécrire en termes de $q_{i, j}$ en utilisant la formule (1.7), et réciproquement en utilisant la formule (1.8).

\subsubsection{Un mot sur la gestion des multiplicités}

Dans ce qui précède, on a toujours supposé pour simplifier que $\varphi_{1}>\cdots>\varphi_{d}$, c'est-à-dire si l'on préfère qu'il n'y a aucun point $(q, \mu)$ qui appartient simultanément aux graphes du plusieurs fonctions $\varphi_{i}$. Dans le cas où cette condition n'est pas réalisée, des complications techniques apparaissent. En particulier, la définition des $q_{i, j}$ et $\mu_{i, j}$ n'est plus correcte et doit être remplacée par :

- le nombre $q_{i, j}$ est la borne inférieure des nombres réels $q$ tels que soit $\psi_{j} \circ \varphi_{i}(q)>q$, soit $\psi_{j} \circ \varphi_{i}(q)=$ $q$ et

$$
\operatorname{Card}\left\{i^{\prime} \leqslant i \mid \varphi_{i^{\prime}}(q)=\mu\right\} \leqslant \operatorname{Card}\left\{j^{\prime} \leqslant j \mid \psi_{j^{\prime}}(\mu)=q\right\}
$$

où on a posé $\mu=\varphi_{i}(q)$;

- le nombre $\mu_{i, j}$ est la borne inférieure des nombres réels $\mu$ tels que soit $\varphi_{i} \circ \psi_{j}(\mu)>\mu$, soit $\varphi_{i} \circ$ $\psi_{j}(\mu)=\mu$ et l'inégalité $(1.13)$ est vérifiée avec $q=\psi_{j}(\mu)$. 
Dans le cas où $\varphi_{1}>\cdots>\varphi_{d}$, on retrouve bien la définition donnée auparavant. En effet, il y a alors un unique indice $i^{\prime}$ (resp. $j^{\prime}$ ) tel que $\mu=\varphi_{i^{\prime}}(q)$ (resp. $q=\psi_{j^{\prime}}(\mu)$ ) à savoir $i^{\prime}=i$ (resp. $j^{\prime}=j$ ), et donc la condition supplémentaire est toujours vérifiée. L'idée dans cette définition est que si, étant donné un couple $(q, \mu)$, on note $i_{1}<i_{2}<\cdots<i_{k}$ et $j_{1}<j_{2}<\cdots<j_{k}$ les entiers tels que :

$$
\varphi_{i_{1}}(q)=\varphi_{i_{2}}(q)=\cdots=\varphi_{i_{k}}(q)=\mu \quad \text { et } \quad \psi_{j_{1}}(\mu)=\psi_{j_{2}}(\mu)=\cdots=\psi_{j_{k}}(\mu)=q
$$

alors on a bien $\psi_{j_{s}} \circ \varphi_{i_{s}}(q)=q$ mais, si $t<s$ le nombre $\psi_{j_{t}} \circ \varphi_{i_{s}}(q)$ doit être considéré comme infinitésimalement plus petit que $q$ et, en tout cas, ne doit pas être pris en compte dans la définition de $q_{i_{s}, j_{t}}$. En formalisant cette vision des choses et en reprenant les arguments développés précédemment, on peut montrer avec un peu de présévérance (exercice laissé au lecteur) que la proposition 1.7 est encore vraie pour tous les $\varphi \in \Phi$ à condition de prendre la définition modifiée précédente. De même, la formule (1.4) demeure, ainsi que les relations 1.5$)$, (1.6) et (1.12).

\subsubsection{Une bijection}

À partir de maintenant, on va faire varier les éléments $\varphi$ dans $\Phi$. C'est pourquoi, afin de lever tout risque d'ambiguïté, on notera dans la suite $q_{i, j}(\varphi)$ et $\mu_{i, j}(\varphi)$ respectivement à la place de $q_{i, j}$ et $\mu_{i, j}$. Soit $I$ l'ensemble des couples $(i, j)$ tels que $1 \leqslant i \leqslant j \leqslant d$. On considère l'espace vectoriel $\left(\mathbb{R}^{2}\right)^{I}$ des suites $\left(q_{i, j}, \mu_{i, j}\right)$ indicées par les éléments de $I$. Soit $K$ le sous-ensemble convexe de $\left(\mathbb{R}^{2}\right)^{I}$ définis par les relations (1.5), (1.6) et 1.12.

Théorème 1.10. L'application

$$
\Phi \rightarrow K, \quad \varphi \mapsto\left(q_{i, j}(\varphi), \mu_{i, j}(\varphi)\right)_{(i, j) \in I}
$$

est une bijection et son inverse est donné par la formule (1.4).

Démonstration. Il s'agit de démontrer qu'étant donné $\left(q_{i, j}, \mu_{i, j}\right) \in K$, la formule (1.4) définit un $d$-uplet $\varphi=\left(\varphi_{1}, \ldots, \varphi_{d}\right)$ qui appartient à $\Phi$ et qui est tel que $q_{i, j}(\varphi)=q_{i, j}$ et $\mu_{i, j}(\varphi)=\mu_{i, j}$ pour tout $(i, j) \in I$. Les conditions 2 et 3 qui définissent l'ensemble $\Phi$ (voir page 11) ne posent aucun problème à part peutêtre en ce qui concerne la croissance des $\varphi_{i}$, mais celle-ci résulte directement des égalités et des inégalités supposées sur les $q_{i, j}$ et $\mu_{i, j}$. On a en outre les inégalités suivantes qui seront utiles dans la suite :

$$
\begin{array}{ll}
\forall q<q_{i, j}, & \varphi_{i}(q) \leqslant b\left(q-q_{i, j}\right)+\mu_{i+1, j+1} \\
\forall q \geqslant q_{i, j}, & \varphi_{i}(q) \geqslant b\left(q-q_{i, j}\right)+\mu_{i, j}
\end{array}
$$

On montre à présent la condition 1 , c'est-à-dire que pour tout indice $i \in\{1, \ldots, d-1\}$, on a $\varphi_{i} \geqslant \varphi_{i+1}$. Soit $q \in \mathbb{R}$. Si $q<q_{i+1, d}$, on a bien $\varphi_{i}(q) \geqslant \varphi_{i+1}(q)=-\infty$. Sinon, il existe $j$ tel que $q_{i+1, j} \leqslant q<q_{i+1, j-1}$. On a alors $\varphi_{i+1}(q)=b\left(q-q_{i+1, j}\right)+\mu_{i+1, j} \leqslant b\left(q-q_{i, j-1}\right)+\mu_{i, j-1} \leqslant \varphi_{i}(q)$ la première inégalité résultant des hypothèses $q_{i+1, j} \geqslant q_{i, j-1}$ et $\mu_{i+1, j} \leqslant \mu_{i, j-1}$, et la seconde résultant de 1.15 ) après avoir remarqué que $q \geqslant q_{i+1, j} \geqslant q_{i, j-1}$.

On en vient à la condition 4. Bien sûr, on prend les fonctions $\psi_{j}$ définies par la formule (1.4). Les inégalités supposées impliquent de même que précédemment qu'elles sont strictement croissantes et rangées par ordre croissant. Soit $(q, \mu)$ un couple de nombres réels. Par définition, un indice $i \in\{1, \ldots, d\}$ vérifie $\mu=\varphi_{i}(q)$ si, et seulement s'il existe $j \in\{1, \ldots, d\}$ tel que

$$
q_{i, j} \leqslant q<q_{i, j-1} \quad \text { et } \quad \mu-\mu_{i, j}=b\left(q-q_{i, j}\right)
$$

De plus, si un tel indice $j$ existe, il est clair qu'il est unique. Ainsi, il existe autant d'indices $i$ satisfaisant $\mu=\varphi_{i}(q)$ que de couples $(i, j)$ satisfaisant (1.16). De même, on démontre qu'il existe autant d'indices $j$ satisfaisant $q=\psi_{j}(\mu)$ que de couples $(i, j)$ satisfaisant :

$$
\mu_{i, j} \leqslant \mu<\mu_{i+1, j} \quad \text { et } \quad \mu-\mu_{i, j}=b\left(q-q_{i, j}\right) .
$$

Il suffit donc de montrer que les conditions 1.16 et 1.17 sont équivalentes, ce qui résulte sans peine de l'égalité (1.6).

Il reste enfin à démontrer que $q_{i, j}(\varphi)=q_{i, j}$ et $\mu_{i, j}(\varphi)=\mu_{i, j}$. Grâce à la relation (1.7), il suffit de démontrer l'égalité pour les $q_{i, j}$. Comme précédemment, on n'écrit la preuve que dans le cas où $\varphi_{1}>$ 
$\cdots>\varphi_{d}$. Il faut alors montrer que pour tout $(i, j) \in I$, on a $\psi_{j} \circ \varphi_{i}\left(q_{i, j}\right) \geqslant q_{i, j}$ et $\psi_{j} \circ \varphi_{i}(q)<q$ pour tout $q<q_{i, j}$. La formule (1.15) montre que $\varphi_{i}\left(q_{i, j}\right) \geqslant \mu_{i, j}$, tandis que, de manière analogue, on démontre que $\psi_{j}\left(\mu_{i, j}\right) \geqslant q_{i, j}$. Il en résulte, en utilisant la croissance, que $\psi_{j} \circ \varphi_{i}\left(q_{i, j}\right) \geqslant \psi_{j}\left(\mu_{i, j}\right) \geqslant q_{i, j}$. Soit $q<q_{i, j}$. Si $\varphi_{i}(q)=-\infty$, il n'y a rien à démontrer; on suppose donc que ce n'est pas le cas. La formule 1.14) implique que $\varphi_{i}(q)<\mu_{i+1, j+1} \leqslant \mu_{i, j}$. Comme, par ailleurs, sur l'intervalle $]-\infty, \mu_{i, j}[$, on a $\psi_{j}(\mu) \leqslant b^{-1}\left(\mu-\mu_{i, j}\right)+q_{i-1, j-1}$, on obtient :

$$
\psi_{j} \circ \varphi_{i}(q) \leqslant q-\left(q_{i, j}-q_{i-1, j-1}\right)-\frac{\mu_{i, j}-\mu_{i+1, j+1}}{b} .
$$

Si l'inégalité est stricte, la démonstration est terminée. Sinon, cela signifie que toutes les inégalités utilisées sont des égalités, et donc en particulier que $\varphi_{i}(q)=b\left(q-q_{i, j}\right)+\mu_{i+1, j+1}$ et que $q_{i, j}-q_{i-1, j-1}=\mu_{i, j}-$ $\mu_{i+1, j+1}=0$ puisque ces deux différences sont toujours positives ou nulles. On applique alors l'inégalité (1.14) au couple $(i-1, j-1)$ : cela donne $\varphi_{i-1}(q) \leqslant b\left(q-q_{i-1, j-1}\right)+\mu_{i, j}=b\left(q-q_{i, j}\right)+\mu_{i, j}=\varphi_{i}(q)$, ce qui contredit l'hypothèse.

On rappelle que l'on avait défini un sous-ensemble $\Phi_{\mathbb{Z}}$ de $\Phi$ caractérisé par certaines conditions dites d'intégrité (les conditions 5 et 6 , page 11). La proposition suivante montre que ce sous-ensemble est facilement caractérisable à l'aide de la bijection du théorème précédent.

Proposition 1.11. Pour tout $\varphi \in \Phi$, les trois conditions suivantes sont équivalentes :

i) $\varphi \in \Phi_{\mathbb{Z}}$

ii) pour tout $(i, j) \in I, q_{i, j}(\varphi) \in \frac{1}{b} \mathbb{Z}$, et pour tout $i \in\{1, \ldots, d\}, q_{i, d}(\varphi) \in \mathbb{Z}$;

iii) pour tout $(i, j) \in I, \mu_{i, j}(\varphi) \in \mathbb{Z}$ et pour tout $i \in\{1, \ldots, d\}$, la somme $\mu_{i, i}(\varphi)+\mu_{i, i+1}(\varphi)+\cdots+$ $\mu_{i, d}(\varphi)$ est divisible par $b-1$.

Démonstration. L'équivalence entre les conditions $i i)$ et $i i i)$ résulte directement des formules (1.7) et (1.8) qui permettent d'exprimer les $\mu_{i, j}$ en fonction des $q_{i, j}$ et réciproquement. Reste donc à montrer l'équivalence entre $i$ ) et $i$ ). À partir de la proposition 1.7, on déduit que ii) implique $i$ ). Si l'on suppose maintenant que $\varphi \in \Phi_{\mathbb{Z}}$, alors toutes les composées $\psi_{j} \circ \varphi_{i}$ sont constantes sur les intervalles de la forme $\left[v, v+\frac{1}{b}\right.$ [ pour $v \in \frac{1}{b} \mathbb{Z}$ et de là, en revenant à la définition, on déduit que les $q_{i, j}(\varphi)$ appartiennent tous à $\frac{1}{b} \mathbb{Z}$. Finalement, il est clair que $q_{i, d}(\varphi)$ est entier pour tout $i$, puisque celui-ci est égal à $q_{i}$ qui est justement supposé entier.

\subsection{Les variétés $\mathcal{X}_{\varphi}$ et leurs dimensions}

Étant donné un $d$-uplet $\tilde{\varphi}=\left(\tilde{\varphi}_{1}, \ldots, \tilde{\varphi}_{d}\right)$, on définit $\tilde{\mathcal{X}}_{\tilde{\varphi}}(k)$ comme l'ensemble des réseaux $L \subset M$ tels que $\tilde{\varphi}_{i}(L)=\tilde{\varphi}_{i}$ pour tout $i$. Si l'on note $\varphi$ le $d$-uplet de fonctions $\varphi_{i}: \mathbb{R} \rightarrow \mathbb{R} \cup\{-\infty\}$ obtenu à partir de $\tilde{\varphi}$ après réordonnement et prolongement, les propositions 1.2 et 1.7 montrent ensemble que $\tilde{\mathcal{X}}_{\tilde{\varphi}}(k)$ est inclus dans $\mathcal{X}_{\mu}(k)$ pour $\mu=\left(\mu_{1,1}(\varphi), \mu_{1,2}(\varphi), \ldots, \mu_{1, d}(\varphi)\right)$. De façon plus précise, on démontre comme dans le lemme 4.2 de [16] que l'on obtient ce faisant une sous-variété $\tilde{\mathcal{X}}_{\tilde{\varphi}}$ de $\mathcal{X}_{\mu}$ qui est localement fermée.

De façon similaire, étant donné $\varphi \in \Phi_{\mathbb{Z}}$, on note $\mathcal{X}_{\varphi}(k)$ l'ensemble des réseaux $L \subset M$ tels que $\varphi(L)=\varphi$; cet ensemble s'écrit manifestement comme une union de $\tilde{\mathcal{X}}_{\tilde{\varphi}}(k)$. Le lemme suivant montre qu'il s'agit même d'une union finie et, par voie de conséquence, que $\mathcal{X}_{\varphi}(k)$ est aussi l'ensemble des $k$ points d'une sous-variété algébrique localement fermée de $\mathcal{X}_{\mu}$ pour le même $\mu$ que précédemment.

Lemme 1.12. On fixe un élément $\varphi \in \Phi_{\mathbb{Z}}$. Alors, il n'existe qu'un nombre fini de d-uplets de fonctions $\left(\tilde{\varphi}_{1}, \ldots, \tilde{\varphi}_{d}\right)$ qui satisfont aux conditions de la proposition 1.2 et qui redonnent le d-uplet $\varphi$ après réordonnement.

Démonstration. Si $\left(\tilde{\varphi}_{1}, \ldots, \tilde{\varphi}_{d}\right)$ est un tel $d$-uplet, alors il existe une permutation $w \in \mathfrak{S}_{d}$ telle que, pour tout $i$, on ait $\tilde{\varphi}_{i}(v)=-\infty$ pour $v<q_{w(i), d}(\varphi)$ et $\tilde{\varphi}_{i}(v)=b v-q_{w(i), d}(\varphi)$ pour $v$ suffisamment grand. Du fait que les fonctions $\tilde{\varphi}_{i}$ doivent en outre être croissantes, on déduit que l'égalité $\tilde{\varphi}_{i}(v)=b v-q_{w(i), d}(\varphi)$ vaut pour tout $v \geqslant q_{d, d}(\varphi)$. Après cela, il ne reste plus qu'un nombre fini de $v$ et donc qu'un nombre fini de possibilités pour attribuer les valeurs manquantes aux $\tilde{\varphi}_{i}$ puisque pour chaque $v$, on ne peut que permuter les nombres $\varphi_{i}(v)$ et on a donc au maximum $d$ ! possibilités. 


\subsubsection{La fonction dimension sur $\Phi$}

Définition 1.13. Soit Leb la mesure de Lebesgue sur $\mathbb{R}$. Si $\varphi=\left(\varphi_{1}, \ldots, \varphi_{d}\right) \in \Phi$, on pose :

$$
\operatorname{dim}(\varphi)=\sum_{1 \leqslant i<i^{\prime} \leqslant d} \operatorname{Leb}\left(\varphi_{i^{\prime}}(\mathbb{R}) \backslash \varphi_{i}(\mathbb{R})\right)
$$

où, si $E$ et $E^{\prime}$ sont des ensembles, on note $E \backslash E^{\prime}$ l'ensemble des éléments qui appartiennent à $E$ mais pas à $E^{\prime}$.

Remarque 1.14. Pour $\varphi=\left(\varphi_{1}, \ldots, \varphi_{d}\right) \in \Phi$, il est clair qu'il existe des constantes $A$ et $B$ telles que $\left[A,+\infty\left[\subset \varphi_{i}(\mathbb{R}) \subset\left[B,+\infty\left[\right.\right.\right.\right.$ pour tout $i$. On en déduit que les différences $\varphi_{i^{\prime}}(\mathbb{R}) \backslash \varphi_{i}(\mathbb{R})$ sont toutes incluses dans l'intervalle $[A, B]$ et donc, en particulier, qu'elles ont une mesure finie. Ainsi $\operatorname{dim}(\varphi)$ est toujours fini.

Via la bijection de la proposition 1.2, un élément $\varphi \in \Phi$ est entièrement déterminé par la donnée des $q_{i, j}(\varphi)$ et $\mu_{i, j}(\varphi)$. Ainsi, le nombre $\operatorname{dim}(\varphi)$ que l'on vient de définir doit s'exprimer en fonction des $q_{i, j}(\varphi)$ et $\mu_{i, j}(\varphi)$. On a plusieurs possibilités pour cela, comme le montre le lemme suivant.

Lemme 1.15. Pour tout $\varphi \in \Phi$, on $a$ :

$$
\begin{aligned}
\operatorname{dim}(\varphi) & =\sum_{j=1}^{d}(d+1-j) \cdot \mu_{1, j}(\varphi)-\sum_{(i, j) \in I} \mu_{i, j}(\varphi) \\
& =b \cdot \sum_{(i, j) \in I} q_{i, j}(\varphi)+\sum_{i=1}^{d}(2 i-1-d-b i) \cdot q_{i, d}(\varphi)
\end{aligned}
$$

Remarque 1.16. On constate en particulier — et ce sera crucial dans la suite - que $\operatorname{dim}(\varphi)$ dépend de façon linéaire des $\mu_{i, j}(\varphi)$ et $q_{i, j}(\varphi)$.

Démonstration. Comme d'habitude, on ne donne la démonstration que dans le cas où $\varphi_{1}>\cdots>\varphi_{d}$. On fixe un indice $i \in\{1, \ldots, d\}$. D'après la proposition 1.7, l'image de la fonction $\varphi_{i}$ s'écrit :

$$
\varphi_{i}(\mathbb{R})=\bigsqcup_{i \leqslant j \leqslant d}\left[\mu_{i, j}, \mu_{i+1, j}[.\right.
$$

Comme on a en outre $\mu_{i+1, j+1} \leqslant \mu_{i, j}$, les intervalles qui apparaissent dans l'union précédente sont « rangés par ordre décroissant $\gg$. On en déduit que le réel $\mu$ n'est pas dans l'image de $\varphi_{i}$ si, et seulement s'il existe $j \in\{i, \ldots, d\}$ tel que $\mu_{i+1, j+1} \leqslant \mu<\mu_{i, j}$ où on a posé par convention $\mu_{i+1, d+1}=-\infty$. Pour un tel $\mu$, on se propose de compter le nombre d'indices $i^{\prime}>i$ tels que $\mu$ appartienne à l'image de $\varphi_{i^{\prime}}$. Comme les fonctions $\varphi_{i^{\prime}}$ sont injectives sur l'intervalle où elles prennent des valeurs finies et que l'on a supposé $\varphi_{1}>\cdots>\varphi_{d}$, cela revient encore à compter le nombre de $q$ pour lesquels il existe $i^{\prime}>i$ tel que $\mu=\varphi_{i^{\prime}}(q)$. Cette condition se réécrit encore :

$$
\exists i^{\prime} \in\{1, \ldots, d\}, \quad \mu=\varphi_{i^{\prime}}(q) \text { et } \varphi_{i+1}(q) \geqslant \mu
$$

puis, d'après la définition des $\psi_{j}$ :

$$
\exists j^{\prime} \in\{1, \ldots, d\}, \quad q=\psi_{j^{\prime}}(\mu) \text { et } \varphi_{i+1}(q) \geqslant \mu .
$$

En remplaçant $q$ par $\psi_{j^{\prime}}(\mu)$, la dernière inégalité devient $\varphi_{i+1} \circ \psi_{j^{\prime}}(\mu) \geqslant \mu$, ce qui équivaut encore à $\mu \geqslant \mu_{i+1, j^{\prime}}$. Au final, on cherche donc à dénombrer les réels $q$ s'écrivant sous la forme $\psi_{j^{\prime}}(\mu)$ pour un indice $j^{\prime}$ tel que $\mu \geqslant \mu_{i+1, j^{\prime}}$. De l'hypothèse supplémentaire $\varphi_{1}>\cdots>\varphi_{d}$, on déduit facilement que $\psi_{1}<\cdots<\psi_{d}$, d'où il suit que le nombre cherché est aussi le nombre d'indices $j^{\prime}$ tels que $\mu \geqslant \mu_{i+1, j^{\prime}}$. Or, comme $\mu$ a été pris dans l'intervalle $\left[\mu_{i+1, j+1}, \mu_{i, j}\right.$ [ et donc a fortiori dans $\left[\mu_{i+1, j+1}, \mu_{i+1, j}[\right.$, on déduit de la décroissance de la suite $j^{\prime} \mapsto \mu_{i+1, j^{\prime}}$ que les $j^{\prime}$ convenables sont ceux de l'ensemble $\{j+1, \ldots, d\}$. En particulier, il y en a $d-j$. 
En résumé, on vient de montrer que le complémentaire de l'image des $\varphi_{i}$ est la réunion disjointe des intervalles $\left[\mu_{i+1, j+1}, \mu_{i, j}\right.$ [ pour $j$ variant dans $\{i, \ldots, d\}$, et que si $\mu \in\left[\mu_{i+1, j+1}, \mu_{i, j}\right.$ [ pour un certain $j$, il y a exactement $d-j$ indices $i^{\prime}>i$ tels que $\mu \in \varphi_{i^{\prime}}(\mathbb{R})$. Il en résulte que

$$
\sum_{i^{\prime}=i+1}^{d} \operatorname{Leb}\left(\varphi_{i^{\prime}}(\mathbb{R}) \backslash \varphi_{i}(\mathbb{R})\right)=\sum_{j=i}^{d-1}(d-j) \cdot\left(\mu_{i, j}-\mu_{i+1, j+1}\right) .
$$

En sommant ces égalités pour tout $i$, il vient :

$$
\operatorname{dim}(\varphi)=\sum_{1 \leqslant i \leqslant j<d}(d-j) \cdot\left(\mu_{i, j}-\mu_{i+1, j+1}\right) .
$$

Les formules annoncées dans la proposition s'en déduisent (avec un peu de calcul) à partir de la relation (1.7).

Corollaire 1.17. Pour tout $\varphi \in \Phi_{\mathbb{Z}}$, on a la congruence :

$$
\operatorname{dim}(\varphi) \equiv-\sum_{j=1}^{d} j \cdot \mu_{1, j}(\varphi) \quad(\bmod b-1)
$$

Démonstration. C'est une conséquence immédiate de la première égalité de la proposition précédente et de la proposition 1.11 .

Le corollaire est intéressant notamment car, dans le cas où $\varphi$ provient d'un réseau $L$, les nombres $\mu_{1, j}(\varphi)$ s'interprètent comme les exposants des diviseurs élémentaires du module engendré par $\sigma(L)$ par rapport à $L$. Par ailleurs, comme on peut s'y attendre, la fonction dim que l'on vient de définir est liée de près à la dimension des variétés $\mathcal{X}_{\varphi}$. Plus précisément, on a le théorème suivant.

Théorème 1.18. Soit $\varphi=\left(\varphi_{1}, \ldots, \varphi_{d}\right) \in \Phi_{\mathbb{Z}}$. Alors

- si $h \neq 0$, on a $\operatorname{dim}_{k} \mathcal{X}_{\varphi}=\operatorname{dim}(\varphi)$;

- si $h=0$, on $a \operatorname{dim}(\varphi) \leqslant \operatorname{dim}_{k} \mathcal{X}_{\varphi} \leqslant \operatorname{dim}(\varphi)+\frac{d(d-1)}{2}$.

\subsubsection{Démonstration du théorème 1.18}

On fixe un élément $\varphi=\left(\varphi_{1}, \ldots, \varphi_{d}\right) \in \Phi_{\mathbb{Z}}$. Puisque $\mathcal{X}_{\varphi}$ s'écrit comme l'union finie des $\tilde{\mathcal{X}}_{\tilde{\varphi}}$ sur les $d$ uplets $\tilde{\varphi}=\left(\tilde{\varphi}_{1}, \ldots, \tilde{\varphi}_{d}\right)$ vérifiant les conditions de la proposition 1.2 et redonnant $\varphi$ après réordonnement, il suffit de démontrer

- d'une part, que la dimension de toutes les variétés $\tilde{\mathcal{X}}_{\tilde{\varphi}}$ est majorée par $\operatorname{dim}(\varphi)$ dans le cas où $h \neq 0$ et $\operatorname{par} \operatorname{dim}(\varphi)+\frac{d(d-1)}{2}$ dans le cas contraire, et

- d'autre part, qu'il existe un $d$-uplet $\tilde{\varphi}$ particulier pour lequel $\operatorname{dim}_{k} \tilde{\mathcal{X}}_{\tilde{\varphi}} \geqslant \operatorname{dim}(\varphi)$.

Pour cela, on suit la méthode de [16].

Notion de famille correcte On fixe des $d$-uplet $\varphi$ et $\tilde{\varphi}$ comme précédemment, et on définit les ensembles suivants :

$$
\begin{gathered}
Q=\frac{1}{b} \mathbb{Z} \times\{1, \ldots, d\} \quad ; \quad \tilde{V}_{\mu}=\left\{(q, i) \in Q \mid \tilde{\varphi}_{i}(q)=\mu\right\} \quad(\operatorname{pour} \mu \in \mathbb{Z}) \quad ; \quad \tilde{V}=\bigcup_{\mu \in \mathbb{Z}} \tilde{V}_{\mu} \\
A=\left\{\left(q, i, q^{\prime}, i^{\prime}\right) \in Q^{2} \mid\left(q^{\prime}, i^{\prime}\right)>(q, i) \text { et } \varphi_{i}\left(q-\frac{1}{b}\right)<\varphi_{i^{\prime}}\left(q^{\prime}\right)<\varphi_{i}(q)\right\} \\
\tilde{A}=\left\{\left(q, i, q^{\prime}, i^{\prime}\right) \in Q^{2} \mid\left(q^{\prime}, i^{\prime}\right)>(q, i) \text { et } \tilde{\varphi}_{i}\left(q-\frac{1}{b}\right)<\tilde{\varphi}_{i^{\prime}}\left(q^{\prime}\right)<\tilde{\varphi}_{i}(q)\right\}
\end{gathered}
$$

et, enfin, pour tout $(q, i) \in \tilde{V}$ :

$$
\tilde{A}(q, i)=\left\{\left(q^{\prime}, i^{\prime}\right) \in Q \mid\left(q^{\prime}, i^{\prime}\right)>(q, i) \text { et } \tilde{\varphi}_{i}\left(q-\frac{1}{b}\right)<\tilde{\varphi}_{i^{\prime}}\left(q^{\prime}\right)<\tilde{\varphi}_{i}(q)\right\} .
$$

Lemme 1.19. On a $\operatorname{Card} \tilde{A} \leqslant \operatorname{Card} A=\operatorname{dim}(\varphi)$. 
Démonstration. On remarque dans un premier temps que si $\left(q, i, q^{\prime}, i^{\prime}\right)$ est un élément de $A$, alors on a nécessairement $i<i^{\prime}$. En effet, on déduit de $\left(q, i, q^{\prime}, i^{\prime}\right) \in A$ que $\varphi_{i}(q)>\varphi_{i^{\prime}}\left(q^{\prime}\right) \geqslant \varphi_{i^{\prime}}(q)$, ce qui ne peut se produire si $i^{\prime} \leqslant i$ étant donné que $\varphi_{1} \geqslant \cdots \geqslant \varphi_{d}$ par hypothèse. On déduit en particulier de cette propriété que, dans la définition de $A$, on peut remplacer l'inégalité $\left(q^{\prime}, i^{\prime}\right)>(q, i)$ par la condition plus simple $q^{\prime} \geqslant q$.

Pour tout $q \in \frac{1}{b} \mathbb{Z}$, il existe une permutation $\tau_{q} \in \mathfrak{S}_{d}$ telle que $\tilde{\varphi}_{i}(q)=\varphi_{\tau_{q}(i)}(q)$ pour tout indice $i$. Pour démontrer que Card $\tilde{A} \leqslant \operatorname{Card} A$, il suffit donc de montrer que, pour tout triplet $\left(q, q^{\prime}, i^{\prime}\right) \in \frac{1}{b} \mathbb{Z} \times Q$ avec $q^{\prime} \geqslant q$, il n'y a pas plus d'indices $i$ tels que $\left(q, i, q^{\prime}, i^{\prime}\right) \in \tilde{A}$ que d'indices $i$ tels que $\left(q, i, q^{\prime}, \tau_{q^{\prime}}\left(i^{\prime}\right)\right) \in A$. D'après le résultat du premier alinéa de la démonstration, il suffit pour cela de démontrer que, si on a posé $\mu=\tilde{\varphi}_{i^{\prime}}\left(q^{\prime}\right)=\varphi_{\tau_{q^{\prime}}\left(i^{\prime}\right)}\left(q^{\prime}\right)$, les deux ensembles suivants :

$$
\tilde{B}=\left\{i \mid \tilde{\varphi}_{i}\left(q-\frac{1}{b}\right)<\mu<\tilde{\varphi}_{i}(q)\right\} \quad \text { et } \quad B=\left\{i \mid \varphi_{i}\left(q-\frac{1}{b}\right)<\mu<\varphi_{i}(q)\right\}
$$

ont même cardinal. Or, on peut écrire

$$
\tilde{B}=\tilde{B}_{1} \backslash \tilde{B}_{2} \quad \text { avec } \quad \tilde{B}_{1}=\left\{i \mid \mu<\tilde{\varphi}_{i}(q)\right\} \quad \text { et } \quad \tilde{B}_{2}=\left\{i \mid \mu \leqslant \tilde{\varphi}_{i}\left(q-\frac{1}{b}\right)\right\}
$$

et de même $B=B_{1} \backslash B_{2}$ où $B_{1}$ et $B_{2}$ sont définis de manière analogue en remplaçant $\tilde{\varphi}_{i}$ par $\varphi_{i}$. On a alors les inclusions $\tilde{B}_{2} \subset \tilde{B}_{1}$ et $B_{2} \subset B_{1}$ alors que, par ailleurs, la permutation $\tau_{q}$ (resp. $\tau_{q-\frac{1}{b}}$ ) induit une bijection de $\tilde{B}_{1}$ dans $B_{1}$ (resp. de $\tilde{B}_{2}$ dans $B_{2}$ ). La conclusion s'ensuit.

Il reste à démontrer que Card $A=\operatorname{dim}(\varphi)$. Pour cela, on fixe deux entiers $i$ et $i^{\prime}$ avec $i<i^{\prime}$. L'ensemble différence $\varphi_{i^{\prime}}(\mathbb{R}) \backslash \varphi_{i}(\mathbb{R})$ s'écrit comme une union disjointe d'intervalles de la forme $[\mu, \mu+1$ [ pour certains entiers $\mu$. De plus, si $\mu$ est un tel entier (i.e. si $\mu \in \varphi_{i^{\prime}}(\mathbb{R}) \backslash \varphi_{i}(\mathbb{R})$ ), il existe $q, q^{\prime} \in \frac{1}{b} \mathbb{Z}$ tels que $\varphi_{i^{\prime}}\left(q^{\prime}\right)=\mu$ et $\left(q, i, q^{\prime}, i^{\prime}\right) \in A$ (on rappelle que, dans la définition de l'ensemble $A$, on peut remplacer $\left(q^{\prime}, i^{\prime}\right)>(q, i)$ par $\left.q^{\prime} \geqslant q\right)$. En outre, les rationnels $q$ et $q^{\prime}$ sont uniquement déterminés. À partir de la définition de $\operatorname{dim}(\varphi)$ (voir définition 1.13), on en déduit que $\operatorname{dim}(\varphi)$ compte le nombre de quadruplets $\left(q, i, q^{\prime}, i^{\prime}\right) \in A$ tels que $i<i^{\prime}$, c'est-à-dire le nombre d'éléments de $A$ puisque l'on a démontré que tout $\left(q, i, q^{\prime}, i^{\prime}\right) \in A$ vérifie la condition supplémentaire $i<i^{\prime}$.

On définit $\tilde{q}_{i}$ comme le nombre associé aux fonctions $\tilde{\varphi}_{i}$ et on note $\left(e_{1}, \ldots, e_{d}\right)$ la base canonique de $M$. La première étape de la preuve consiste à démontrer qu'étant donné un réseau $L$ de $M$ tel que $\tilde{\varphi}_{i}(L)=\tilde{\varphi}_{i}$ pour tout $i$, il existe des éléments $v_{q, i} \in M_{k\left(\left(u^{1 / b}\right)\right)}$ pour $(q, i) \in \tilde{V}$ et des élements $a_{q, i, q^{\prime}, i^{\prime}} \in k$ pour $\left(q, i, q^{\prime}, i^{\prime}\right) \in \tilde{A}$ qui vérifient :

i) pour tout $(q, i) \in \tilde{V}$, on a $\operatorname{val}\left(v_{q, i}\right)=(q, i)$ et $\operatorname{val}\left(v_{q, i}-u^{q} e_{i}\right)>(q, i)$;

ii) pour tout $(q, i) \in \tilde{V}$, on a $v_{q, i} \in k\left[\left[u^{1 / b}\right]\right] \otimes_{k[[u]]} L$ et $w_{q, i}=u^{-\tilde{\varphi}_{i}(q)} \sigma\left(v_{q, i}\right) \in L$;

iii) pour tout nombre entier $\mu$, les éléments $\left(w_{q, i} \bmod u\right)$ pour $(q, i)$ parcourant $\tilde{V}_{\mu}$ forment une famille libre sur $k$ dans $L / u L$;

iv) pour tout $(q, i) \in \tilde{V}$ tel que $\left(q-\frac{1}{b}, i\right) \in \tilde{V}$, on a

$$
v_{q, i}=u^{1 / b} v_{q-\frac{1}{b}, i}+\sum_{\left(q^{\prime}, i^{\prime}\right) \in \tilde{A}(q, i)} a_{q, i, q^{\prime}, i^{\prime}} \cdot v_{q^{\prime}, i^{\prime}}
$$

v) pour tout $(q, i) \in \tilde{V}$ tel que $\tilde{\varphi}_{i}(q)=b q-\tilde{q}_{i}$ (ou de façon équivalente pour un tel $(q, i)$ ), on a

$$
v_{\tilde{q}_{i}, i}=w_{q, i}+\sum_{\left(q^{\prime}, i^{\prime}\right) \in \tilde{A}\left(q_{i}, i\right)} a_{q_{i}, i, q^{\prime}, i^{\prime}}^{p^{h}} \cdot v_{q^{\prime}, i^{\prime}}-\sum_{\substack{\left(q^{\prime}, i^{\prime}\right) \in \tilde{A}(q, i) \\ q^{\prime}=q}} a_{q, i, q, i^{\prime}}^{p^{h}} \cdot u^{\tilde{q}_{i}} e_{i^{\prime}}
$$

où $w_{q, i}=u^{-\tilde{\varphi}_{i}(q)} \sigma\left(v_{q, i}\right)$ comme ci-dessus.

Une famille $\left(v_{q, i}, a_{q, i, q^{\prime}, i^{\prime}}\right)$ est dite correcte pour $L$ si elle vérifie les conditions précédentes et, étant donné un entier $n$, elle est dite $n$-correcte si elle vérifie i), ii), iii) et si les égalités iv) et v) sont vraies respectivement modulo $u^{q+\frac{n}{b}}$ et $u^{\tilde{q}_{i}+\frac{n}{b}}$. On va construire une famille correcte en procédant par approximations successives. Pour amorcer la construction, on se donne des éléments $v_{q, i}$ vérifiant simplement les conditions i), ii) et iii); leur existence résulte de la définition des fonctions $\tilde{\varphi}_{i}(L)$ et, en ce qui concerne iii), d'une analyse de la démonstration du lemme 4.1 de [16]. On choisit également $a_{q, i, q^{\prime}, i^{\prime}}=0$ pour tout $\left(q, i, q^{\prime}, i^{\prime}\right) \in \tilde{A}$. La famille $\left(v_{q, i}, a_{q, i, q^{\prime}, i^{\prime}}\right)$ est alors 0-correcte. L'étape d'itération est donnée par le lemme suivant duquel il résulte directement l'existence souhaitée après un passage à la limite. 
Lemme 1.20. Soit $\left(v_{q, i}, a_{q, i, q^{\prime}, i^{\prime}}\right)$ une famille $n$-correcte pour $L$ pour un certain entier $n \geqslant 0$. On pose $m=n+1$. Alors, il existe $\left(v_{q, i}^{\prime}, a_{q, i, q^{\prime}, i^{\prime}}^{\prime}\right)$ une famille $m$-correcte pour $L$ telle que

- on ait $v_{q, i}^{\prime} \equiv v_{q, i}\left(\bmod u^{q+\frac{n}{b}}\right)$ pour tout $(q, i) \in \tilde{V}$;

- si $n$ est suffisamment grand, on ait aussi $a_{q, i_{0}, q^{\prime}, i^{\prime}}^{\prime}=a_{q, i_{0}, q^{\prime}, i^{\prime}}$ pour tout $\left(q, i_{0}, q^{\prime}, i^{\prime}\right) \in \tilde{A}$.

Démonstration. On construit les $v_{q, i}^{\prime}$ par récurrence sur $q$ et à $q$ fixé par récurrence descendante sur $i$. Autrement dit, on considère $(q, i) \in \tilde{V}$, on suppose que tous les $v_{q^{\prime}, i^{\prime}}^{\prime} \in \tilde{V}$ avec $q^{\prime}<q$ ou $q^{\prime}=q$ et $i^{\prime}>i$ sont construits et on cherche à construire $v_{q, i}^{\prime}$. On suppose d'abord que $\left(q-\frac{1}{b}, i\right)$ appartient à $\tilde{V}$ et on regarde dans ce cas l'équation 1.18 modulo $u^{q+\frac{m}{b}}$, i.e. la congruence

$$
v_{q, i}^{\prime} \equiv u^{1 / b} \cdot v_{q-\frac{1}{b}, i}^{\prime}+\sum_{\left(q^{\prime}, i^{\prime}\right) \in \tilde{A}(q, i)} a_{q, i, q^{\prime}, i^{\prime}}^{\prime} \cdot v_{q^{\prime}, i^{\prime}}^{\prime} \quad\left(\bmod u^{q+\frac{m}{b}}\right)
$$

qui doit être satisfaite par l'élément $v_{q, i}^{\prime}$ que l'on veut construire. Dans l'expression précédente, les $v_{q-\frac{1}{5}, i}^{\prime}$ ont déjà été construits de même que les $v_{q^{\prime}, i^{\prime}}^{\prime}$ pour $q=q^{\prime}$ car on a alors nécessairement $i^{\prime}>i$. Si $q^{\prime}>q$, en revanche, on n'a pas encore construit $v_{q^{\prime}, i^{\prime}}^{\prime}$ mais on souhaite le faire de façon à ce que $v_{q^{\prime}, i^{\prime}}^{\prime} \equiv v_{q^{\prime}, i^{\prime}}$ $\left(\bmod u^{q^{\prime}+\frac{n}{b}}\right)$ et donc a fortiori $v_{q^{\prime}, i^{\prime}}^{\prime} \equiv v_{q^{\prime}, i^{\prime}}\left(\bmod u^{q+\frac{m}{b}}\right)$. On cherche donc à ce que $v_{q^{\prime}, i^{\prime}}^{\prime}$ satisfasse la congruence 1.20) où on a remplacé $v_{q^{\prime}, i^{\prime}}^{\prime}$ pour $q^{\prime}>q$ par $v_{q^{\prime}, i^{\prime}}$. En fait, on va chercher $v_{q^{\prime}, i^{\prime}}^{\prime}$ de sorte que cette nouvelle congruence soit une égalité, c'est-à-dire de sorte que

$$
v_{q, i}^{\prime}=u^{1 / b} \cdot v_{q-\frac{1}{b}, i}^{\prime}+\sum_{\left(q^{\prime}, i^{\prime}\right) \in \tilde{A}(q, i)} a_{q, i, q^{\prime}, i^{\prime}}^{\prime} \cdot x_{q^{\prime}, i^{\prime}}
$$

où, pour unifier les écritures, on a posé, pour $\left(q^{\prime}, i^{\prime}\right) \in \tilde{A}(q, i), x_{q^{\prime}, i^{\prime}}=v_{q^{\prime}, i^{\prime}}^{\prime}$ si $q^{\prime}=q$ et $x_{q^{\prime}, i^{\prime}}=v_{q^{\prime}, i^{\prime}}$ sinon. Par ailleurs, en plus de cela, on doit avoir $\sigma\left(v_{q, i}^{\prime}\right) \in u^{\tilde{\varphi}_{i}(q)} L$. En reportant la valeur désirée de $v_{q, i}^{\prime}$ donnée par l'égalité (1.21), on est amené à démontrer qu'il existe $a_{q, i, q^{\prime}, i^{\prime}}^{\prime} \in k$ tels que :

$$
\sigma\left(u^{1 / b} \cdot v_{q-\frac{1}{b}, i}^{\prime}+\sum_{\left(q^{\prime}, i^{\prime}\right) \in \tilde{A}(q, i)} a_{q, i, q^{\prime}, i^{\prime}}^{\prime} \cdot x_{q^{\prime}, i^{\prime}}\right) \in u^{\tilde{\varphi}_{i}(q)} L
$$

Par définition de $\tilde{\varphi}_{i}(q)$, on sait qu'il existe dans $L$ un élément $x$ de valuation $(q, i)$ tel que $\sigma(x) \in u^{\tilde{\varphi}_{i}(q)}$. Quitte à multiplier $x$ par une constante dans $k$, on peut en outre supposer que val $\left(x-u^{1 / b} v_{q-\frac{1}{b}, i}^{\prime}\right)>(q, i)$. On en déduit, en utilisant la condition i), que $x-u^{1 / b} v_{q-\frac{1}{b}, i}^{\prime}$ s'écrit comme une somme infinie, portant sur tous les couples $\left(q^{\prime}, i^{\prime}\right)$ strictement supérieurs à $(q, i)$, de termes de la forme $a_{q, i, q^{\prime}, i^{\prime}}^{\prime} v_{q^{\prime}, i^{\prime}}$ avec $a_{q, i, q^{\prime}, i^{\prime}}^{\prime} \in k$. Puisque $\sigma\left(x_{q^{\prime}, i^{\prime}}\right) \in u^{\tilde{\varphi}_{i^{\prime}}\left(q^{\prime}\right)} L$, on peut, quitte à changer $x$ en un autre élément de valuation $(q, i)$ tel que $\sigma(x) \in u^{\tilde{\varphi}_{i}(q)}$, retirer de la somme précédente les contributions apportées par les couples $\left(q^{\prime}, i^{\prime}\right)$ tels que $\tilde{\varphi}_{i^{\prime}}\left(q^{\prime}\right) \geqslant \tilde{\varphi}_{i}(q)$. La somme restante est alors finie, car il n'existe de toute façon qu'un nombre fini de couples $\left(q^{\prime}, i^{\prime}\right) \in \tilde{V}$ tels que $\tilde{\varphi}_{i^{\prime}}\left(q^{\prime}\right)<\tilde{\varphi}_{i}(q)$. Pour conclure, il ne reste plus qu'à démontrer que les $a_{q, i, q^{\prime}, i^{\prime}}^{\prime}$ sont nécessairement nuls dès que $\tilde{\varphi}_{i^{\prime}}\left(q^{\prime}\right) \leqslant \tilde{\varphi}_{i}\left(q-\frac{1}{b}\right)$. On part pour cela de la relation

$$
\sigma(x)=u \cdot \sigma\left(v_{q-\frac{1}{b}, i}^{\prime}\right)+\sum_{\substack{\left(q^{\prime}, i^{\prime}\right)>(q, i) \\ \tilde{\varphi}_{i^{\prime}}\left(q^{\prime}\right)<\tilde{\varphi}_{i}(q)}}\left(a_{q, i, q^{\prime}, i^{\prime}}^{\prime}\right)^{p^{h}} \cdot \sigma\left(x_{q^{\prime}, i^{\prime}}\right) \in u^{\tilde{\varphi}_{i}(q)} L .
$$

obtenue simplement en développant. On définit également la valuation $L$-adique $v{ }_{L}(v)$ d'un élément $v \in M$ comme le plus grand entier $n$ tel que $v \in u^{n} L$. La valuation $L$-adique d'un élément de la forme $\sigma\left(v_{q^{\prime}, i^{\prime}}\right)$ ou $\sigma\left(v_{q^{\prime}, i^{\prime}}^{\prime}\right)$ est alors égale à $\tilde{\varphi}_{i^{\prime}}\left(q^{\prime}\right)$ : en effet, elle est supérieure ou égale à cette valeur d'après la condition ii) et l'inégalité ne peut être stricte par définition de $\tilde{\varphi}_{i^{\prime}}\left(q^{\prime}\right)$. Par ailleurs, à partir de la condition iii), il est facile de montrer que la valuation $L$-adique d'une somme de termes de la forme $c_{q^{\prime}, i^{\prime}} \sigma\left(x_{q^{\prime}, i^{\prime}}\right)$ (avec $c_{q^{\prime}, i^{\prime}} \in k$ ) est toujours égale au minimum des valuations $L$-adiques des $c_{q^{\prime}, i^{\prime}} \sigma\left(x_{q^{\prime}, i^{\prime}}\right)$. Ainsi aucun terme de la somme qui apparaît dans 1.23 ne peut avoir une valuation $L$-adique strictement inférieure à $\operatorname{val}_{L}\left(u \cdot \sigma\left(v_{q-\frac{1}{b}, i}^{\prime}\right)\right)=1+\tilde{\varphi}_{i}\left(q-\frac{1}{b}\right)$, ce qui implique finalement ce qu'il fallait démontrer.

Si maintenant, au contraire, $\left(q-\frac{1}{b}, i\right) \notin \tilde{V}$, on raisonne de manière similaire sauf que l'on part désormais de l'équation (1.19) et comme précédemment on remplace $w_{q, i}^{\prime}=u^{-\tilde{\varphi}_{i}(q)} \sigma\left(v_{q, i}^{\prime}\right)$ par $w_{q, i}=$ 
$u^{-\tilde{\varphi}_{i}(q)} \sigma\left(v_{q, i}\right)$. Après cela, il n'est plus difficile de vérifier que $v_{q, i}^{\prime} \equiv v_{q, i}\left(\bmod u^{q+\frac{n}{b}}\right)$ pour tout $(q, i) \in$ $\tilde{V}$ et que la famille $\left(v_{q, i}^{\prime}, a_{q, i, q^{\prime}, i^{\prime}}^{\prime}\right)$ que nous avons construite est bien $m$-correcte. Il reste à montrer que si $n$ est suffisamment grand, on a $a_{q, i, q^{\prime}, i^{\prime}}=a_{q, i, q^{\prime}, i^{\prime}}^{\prime}$ pour tout quadruplet $\left(q, i, q^{\prime}, i^{\prime}\right) \in \tilde{A}$. Mais cela résulte directement du fait que, en vertu des congruences $v_{q, i}^{\prime} \equiv v_{q, i}\left(\bmod u^{q+\frac{n}{b}}\right)$ pour tout $(q, i) \in \tilde{V}$, l'assertion (1.22), de même que son analogue dans le cas où $\left(q-\frac{1}{b}, i\right) \notin \tilde{V}$, est vraie avec $a_{q, i, q^{\prime}, i^{\prime}}^{\prime}=a_{q, i, q^{\prime}, i^{\prime}}$ si $n$ est suffisamment grand.

Remarque 1.21. En reprenant l'argument de la démonstration de l'existence des $a_{q, i, q^{\prime}, i^{\prime}}^{\prime}$, on voit que ceuxci sont en fait uniquement déterminés. Il résulte de cette remarque, par passage à la limite, que les éléments $a_{q, i, q^{\prime}, i^{\prime}}$ dans une famille correcte pour $L$ sont, entièrement déterminés par $L$.

Il est également possible à partir d'une famille correcte $\left(v_{q, i}, a_{q, i, q^{\prime}, i^{\prime}}\right)$ de retrouver le réseau $L$ : en effet, à partir des conditions i) et ii), on démontre directement que $L$ est le module engendré par les $v_{q, i}$ pour $(q, i)$ parcourant $V$. Le lemme suivant montre qu'en fait $L$ est déjà engendré par les $d$ vecteurs $v_{\tilde{q}_{i}, i}$ (qui en forment donc une base).

Lemme 1.22. Soit $\left(v_{q, i}, a_{q, i, q^{\prime}, i^{\prime}}\right)$ une famille correcte pour L. Pour tout $(q, i) \in \tilde{V}$, il existe des $\lambda_{s} \in k[[u]]$ $(1 \leqslant s \leqslant d)$ tels que :

$$
v_{q, i}=\sum_{s=1}^{d} \lambda_{s} \cdot u^{q-\tilde{q}_{s}} v_{\tilde{q}_{s}, s}
$$

et $\lambda_{s}=0$ si $q<\tilde{q}_{s}$ ou si $q=\tilde{q}_{s}$ et $s<i$.

Démonstration. $\mathrm{Si}\left(q-\frac{1}{b}, i\right) \notin \tilde{V}$, alors $q=\tilde{q}_{i}$ et le résultat est clair. Dans le cas contraire, la relation (1.18) assure que $v_{q, i}$ s'exprime en termes de $v_{q-\frac{1}{b}, i}$ et des $v_{q^{\prime}, i^{\prime}}$ pour $\left(q^{\prime}, i^{\prime}\right) \in \tilde{A}(q, i)$. Or, les nombres $\tilde{\varphi}_{i}\left(q-\frac{1}{b}\right)$ et $\varphi_{i^{\prime}}\left(q^{\prime}\right)$ sont tous strictement plus petits que $\varphi_{i}(q)$. Une récurrence sur le nombre $\tilde{\mu}=\tilde{\varphi}_{i}(q)$ permet donc de terminer la démonstration (on remarque que l'initialisation ne pose pas de problème car si $\tilde{\mu}$ est suffisamment petit, aucun couple $(q, i)$ ne convient).

Remarque 1.23. Un examen de la démonstration précédente indique, en outre, que les $\lambda_{s}$ s'expriment uniquement en fonction des $a_{q, i, q^{\prime}, i^{\prime}}$ et des propriétés combinatoires des fonctions $\tilde{\varphi}_{i}$.

L'espace des familles correctes Dans ce paragraphe, on explique comment l'invariant «famille correcte $\gg$ permet de paramétrer les réseaux $L$. À partir de maintenant, nous ne fixe donc plus un réseau $L$ mais, au contraire, on considère l'ensemble $\mathcal{C}(k)$ des familles $\left(v_{q, i}, a_{q, i, q^{\prime}, i^{\prime}}\right)$ satisfaisant les conditions i), iv) et v) précédemment énoncées. Manifestement, $\mathcal{C}(k)$ est l'ensemble des $k$-points d'une variété algébrique définie sur $k$ que l'on note $\mathcal{C}$.

Lemme 1.24. On suppose que $\left(v_{q, i}, a_{q, i, q^{\prime}, i^{\prime}}\right)$ et $\left(v_{q, i}^{\prime}, a_{q, i, q^{\prime}, i^{\prime}}\right)$ (avec les mêmes $\left.a_{q, i, q^{\prime}, i^{\prime}}\right)$ vérifient les conditions $i)$, iv) et $v)$, et que pour tout $i \in\{1, \ldots, d\}$, on a $v_{\tilde{q}_{i}, i} \equiv v_{\tilde{q}_{i}, i}^{\prime}\left(\bmod u^{\tilde{q}_{i}+\frac{1}{b}}\right)$. Alors $v_{q, i}=v_{q, i}^{\prime}$ pour tout $(q, i) \in \tilde{V}$.

Démonstration. On pose, pour simplifier les écritures, $v_{i}=u^{-\tilde{q}_{i}} v_{\tilde{q}_{i}, i}$ et de même $v_{i}^{\prime}=u^{-\tilde{q}_{i}} v_{\tilde{q}_{i}, i}^{\prime}$. L'hypothèse s'écrit alors $v_{i} \equiv v_{i}^{\prime}\left(\bmod u^{1 / b}\right)$. En utilisant l'égalité (1.19), le lemme 1.22 ainsi que la remarque 1.23, on voit qu'il existe des matrices $G$ et $H$ à coefficients dans $k[[u]]$ de taille respectivement $d \times d$ et $1 \times d$ telles que

$$
\left(\sigma\left(v_{1}\right), \ldots, \sigma\left(v_{d}\right)\right)=\left(v_{1}, \ldots, v_{d}\right) G+H \quad \text { et } \quad\left(\sigma\left(v_{1}^{\prime}\right), \ldots, \sigma\left(v_{d}^{\prime}\right)\right)=\left(v_{1}^{\prime}, \ldots, v_{d}^{\prime}\right) G+H .
$$

De plus, on vérifie que la matrice $G$ s'écrit $I_{d}+G^{\prime}$ où $G^{\prime}$ est topologiquement nilpotente; en particulier $G$ est inversible. En posant $w_{i}=v_{i}-v_{i}^{\prime}$, on a par hypothèse $w_{i} \equiv 0\left(\bmod u^{1 / b}\right)$ et, d'après ce qui précède, $\left(\sigma\left(w_{1}\right), \ldots, \sigma\left(w_{d}\right)\right)=\left(w_{1}, \ldots, w_{d}\right) G$. Comme $G$ est inversible, cela implique que $w_{i} \equiv 0(\bmod u)$. En répétant l'argument, on obtient $w_{i} \equiv 0\left(\bmod u^{b^{n}}\right)$ pour tout $n$, c'est-à-dire $w_{i}=0$. Finalement, $v_{i}=v_{i}^{\prime}$ et une nouvelle application du lemme 1.22 permet de conclure.

Remarque 1.25. Le lemme précédent reste vrai si $k$ est remplacé par une $k$-algèbre quelconque. 
Soit $\mathcal{A}=\mathbb{A}_{k}^{\tilde{A}}$ l'espace affine standard sur $k$ dont les coordonnées sont indicées par l'ensemble $\tilde{A}$; c'est une variété algébrique de dimension Card $\tilde{A}$. On dispose par ailleurs d'un morphisme naturel $f: \mathcal{C} \rightarrow \mathcal{A}$ qui à une famille $\left(v_{q, i}, a_{q, i, q^{\prime}, i^{\prime}}\right)$ associe le vecteur de $a_{q, i, q^{\prime}, i^{\prime}}$. Le lemme précédent et la remarque qui le suit montrent que les fibres de $f$ sont de dimension inférieure ou égale à $\frac{d(d-1)}{2}$. En outre, lorsque $h \neq 0$, un examen de la preuve du lemme 1.24 montre même que $f$ est étale. Ainsi, si l'on pose $\varepsilon=1$ si $h=0$ et $\varepsilon=0$ dans le cas contraire, on obtient :

$$
\operatorname{dim}_{k} \mathcal{C} \leqslant \operatorname{dim}_{k} \mathcal{A}+\varepsilon \cdot \frac{d(d-1)}{2}=\operatorname{Card} A+\varepsilon \cdot \frac{d(d-1)}{2} \leqslant \operatorname{dim}(\varphi)+\varepsilon \cdot \frac{d(d-1)}{2} .
$$

Par ailleurs, l'application qui à une famille $\left(v_{q, i}, a_{q, i, q^{\prime}, i^{\prime}}\right) \in \mathcal{C}(k)$ associe le réseau engendré par les $v_{q, i}$ définit un morphisme algébrique $g$ de $\mathcal{C}$ dans la grassmanienne affine sur $k$. Le fait que tout réseau $L$ appartenant à $\tilde{\mathcal{X}}_{\tilde{\varphi}}(k)$ admette une famille correcte signifie que l'image de $g$ contient $\tilde{\mathcal{X}}_{\tilde{\varphi}}$. Ainsi on obtient $\operatorname{dim}_{k} \tilde{\mathcal{X}}_{\tilde{\varphi}} \leqslant \operatorname{dim}_{k} \mathcal{C}$ et la majoration que l'on voulait suit alors de 1.24).

Démonstration de la minoration On se place ici dans le cas où $\tilde{\varphi}_{1} \geqslant \tilde{\varphi}_{2} \geqslant \cdots \geqslant \tilde{\varphi}_{d}$ et on souhaite montrer qu'alors $\operatorname{dim}_{k} \tilde{\mathcal{X}}_{\tilde{\varphi}} \geqslant \operatorname{dim}(\varphi)$. Dans ce cas particulier, les ensembles $A$ et $\tilde{A}$ coïncident et, d'après le lemme 1.19, leur cardinal vaut $\operatorname{dim}(\varphi)$.

Lemme 1.26. Le morphisme $f: \mathcal{C} \rightarrow \mathcal{A}$ défini précédemment est un isomorphisme.

Démonstration. Soit $R$ une $k$-algèbre. Il s'agit de montrer que pour tout $\left(a_{q, i, q^{\prime}, i^{\prime}}\right) \in \mathcal{A}(R)$. Il existe une unique famille $\left(v_{q, i}\right)_{(q, i) \in \tilde{V}}$ d'éléments de $M \otimes_{k((u))} R\left(\left(u^{1 / b}\right)\right)$ telle que $\left(v_{q, i}, a_{q, i, q^{\prime}, i^{\prime}}\right) \in \mathcal{C}(R)$.

On construit les $v_{q, i}$ et on démontre leur unicité par récurrence descendante sur $i$. En reprenant la démonstration du lemme 1.24, on voit que l'élément $v_{i}=u^{-\tilde{q}_{i}} v_{\tilde{q}_{i}, i}$ doit satisfaire une équation de la forme

$$
\sigma\left(v_{i}\right)=v_{i}+\sum_{s=i+1}^{d} \lambda_{s} v_{s}
$$

où les $\lambda_{s}$ sont de valuation $u$-adique strictement positive (les éventuels termes de valuation 0 s'annulent avec le terme $\sum_{\left(q^{\prime}, i^{\prime}\right) \in \tilde{A}(q, i), q^{\prime}=q} a_{q, i, q, i^{\prime}}^{p^{h}} \cdot u^{\tilde{q}_{i}} e_{i^{\prime}}$ de la formule 1.19)). Comme les $v_{s}$ pour $s>i$ sont déjà connus, on a à résoudre une équation de la forme $\sigma\left(v_{i}\right)=v_{i}-c$ où $c$ est un élément connu de valuation strictement positive de $R\left[\left[u^{1 / b}\right]\right]$. Une telle équation a bien une unique solution, à savoir $v_{i}=\sum_{n=0}^{\infty} \sigma^{n}(c)$. Les $v_{q, i}$ pour $q>q_{i}$ s'obtiennent alors à partir d'une variante du lemme 1.22 .

À présent, il est aisé de conclure. La remarque 1.21 montre que le morphisme $g: g^{-1}\left(\tilde{\mathcal{X}}_{\tilde{\varphi}}\right) \rightarrow \tilde{\mathcal{X}}_{\tilde{\varphi}}$ est également un isomorphisme. Il suffit donc de démontrer que $g^{-1}\left(\tilde{\mathcal{X}}_{\tilde{\varphi}}\right)$ est un ouvert non vide de $\mathcal{C}$, ce qui se fait comme dans la preuve du Claim 3 de [16].

\section{Mise en place de la méthode}

Les théorèmes 1.10 et 1.18 permettent de reformuler le problème de calculer — ou disons, plutôt d'estimer - la dimension de $\mathcal{X}_{\leqslant e}$ en un problème de programmation linéaire. Dans cette section, nous mettons en place les outils nécessaires à la résolution de ce dernier problème puis, en guise d'exemple, nous illustrons la méthode proposée en démontrant le théorème 2 de l'introduction sous une hypothèse additionnelle. La démonstration complète de ce théorème est reportée à la section suivante, $\S 3.3 .3$.

\subsection{Préliminaires de programmation linéaire}

On considère un espace euclidien $E$ dont on note $\langle\cdot \mid \cdot\rangle_{E}$ le produit scalaire. On se donne :

- un cône convexe $Q \subset E$, c'est-à-dire un sous-ensemble non vide de $E$ stable par addition et par multiplication par les nombres réels positifs ou nuls ;

- une application linéaire $f: E \rightarrow \mathbb{R}^{n}$ où $n$ est un certain entier naturel;

- une forme linéaire $\ell: E \rightarrow \mathbb{R}$. 
Étant donné que $E$ est un espace euclidien, il existe des vecteurs $\vec{\ell}, \vec{f}_{1}, \ldots, \vec{f}_{n}$ tels que $\ell(x)=\langle x \mid \vec{\ell}\rangle_{E}$ et $f(x)=\left(\left\langle x \mid \vec{f}_{1}\right\rangle_{E}, \ldots,\left\langle x \mid \vec{f}_{n}\right\rangle_{E}\right)$ pour tout $x \in E$. On souhaite étudier la fonction $a_{Q, f, \ell}: \mathbb{R}^{n} \rightarrow$ $\mathbb{R} \cup\{ \pm \infty\}$ définie par :

$$
a_{Q, f, \ell}(y)=\sup _{\substack{x \in Q \\ f(x)=y}} \ell(x)=\sup _{\substack{x \in Q \\\left\langle x \mid \vec{f}_{i}\right\rangle_{E}=y_{i}}}\langle x \mid \vec{\ell}\rangle_{E} \quad \text { où } y=\left(y_{1}, \ldots, y_{n}\right)
$$

en convenant, comme d'habitude, que la borne supérieure de l'ensemble vide est $-\infty$ et celle d'un ensemble non majorée est $+\infty$. Soit $Q^{\star}$ le cône dual de $Q$ :

$$
Q^{\star}=\left\{x \in E \mid\left\langle x \mid x^{\prime}\right\rangle_{E} \geqslant 0, \quad \forall x^{\prime} \in Q\right\} .
$$

Si $Q$ est défini par les inégalités $\left\langle x \mid \vec{v}_{i}\right\rangle_{E} \geqslant 0(1 \leqslant i \leqslant N)$, alors $Q^{\star}$ est le cône convexe engendré par les vecteurs $v_{i}$, c'est-à-dire l'ensemble des vecteurs de la forme $\lambda_{1} \vec{v}_{1}+\cdots+\lambda_{N} \vec{v}_{N}$ pour des scalaires $\lambda_{i} \in \mathbb{R}^{+}$. On introduit $A_{Q, f, \ell}$ l'ensemble convexe défini par :

$$
A_{Q, f, \ell}=\left\{y=\left(y_{1}, \ldots, y_{n}\right) \in \mathbb{R}^{n} \mid\left(y_{1} \vec{f}_{1}+\cdots+y_{n} \vec{f}_{n}\right)-\vec{\ell} \in Q^{\star}\right\} .
$$

Le théorème suivant établit un lien de dualité entre maximisation $\operatorname{sur} Q$ et minimisation sur $A_{Q, f, \ell}$.

Théorème 2.1. Avec les notations précédentes, on $a$ :

$$
a_{Q, f, \ell}(y)=\inf _{\alpha \in A_{Q, f, \ell}}\langle\alpha \mid y\rangle_{n}
$$

où $\langle\cdot \mid \cdot\rangle_{n}$ désigne le produit scalaire usuel sur $\mathbb{R}^{n}$.

Démonstration. Il s'agit un résultat classique de dualité en programmation linéaire. On rappelle quand même brièvement comment on l'établit. On remarque tout d'abord que la fonction $a_{Q, f, \ell}$ est concave. Le théorème de Hahn Banach assure qu'elle s'écrit comme la borne inférieure des fonctions affines qui la majorent. Or un calcul immédiat montre que la fonction affine $\mathbb{R}^{n} \rightarrow \mathbb{R},\left(y_{1}, \ldots, y_{n}\right) \mapsto \alpha_{1} y_{1}+\cdots+$ $\alpha_{n} y_{n}+\beta\left(\alpha_{i}, \beta \in \mathbb{R}\right)$ majore $a_{Q, f, \ell}$ si, et seulement si

$$
\forall x \in Q, \quad\left\langle x \mid \vec{\ell}-\left(\alpha_{1} \vec{f}_{1}+\cdots+\alpha_{n} \vec{f}_{n}\right)\right\rangle_{E} \leqslant \beta .
$$

Comme $Q$ est un cone convexe, ceci est encore équivalent à $\beta \geqslant 0$ et $\left(\alpha_{1} \overrightarrow{f_{1}}+\cdots+\alpha_{n} \overrightarrow{f_{n}}\right)-\vec{\ell} \in Q^{\star}$. Le théorème en résulte.

Pour ce que l'on veut faire, on aura besoin de travailler dans une situation légèrement plus générale que celle qui vient d'être étudiée. Précisément, en plus de $Q, f$ et $\ell$, on se donne maintenant deux cônes convexes $C$ et $D$ inclus dans $\mathbb{R}^{n}$, et on considère la fonction $b_{Q, f, \ell, C, D}: \mathbb{R}^{n} \rightarrow \mathbb{R} \cup\{ \pm \infty\}$ définie par :

$$
\begin{aligned}
b_{Q, f, \ell, C, D}(y) & =\sup _{x \in Q, f(x) \in y-C} \ell(x) & & \text { si } y \in D \\
& =-\infty & & \text { sinon }
\end{aligned}
$$

où par définition $y-C$ est l'ensemble des vecteurs $y^{\prime} \in \mathbb{R}^{n}$ pour lesquels il existe $c \in C$ tel que $y-c=y^{\prime}$, ou autrement dit $y-y^{\prime} \in C$. Dans la suite, lorsque $D=\mathbb{R}^{n}$, on s'autorisera à ne pas le noter en indice. On note $C^{\star}$ et $D^{\star}$ les cônes duaux respectifs de $C$ et $D$. On pose :

$$
B_{Q, f, \ell, C, D}=\left(A_{Q, f, \ell} \cap C^{\star}\right)+D^{\star}
$$

où la notation précédente signifie que les éléments de $B_{Q, f, \ell, C, D}$ sont ceux qui s'écrivent sous la forme $y_{1}+y_{2}$ avec $y_{1} \in A_{Q, f, \ell} \cap C^{\star}$ et $y_{2} \in D^{\star}$.

Proposition 2.2. Avec les notations précédentes, on a :

$$
b_{Q, f, \ell, C, D}(y)=\inf _{\alpha \in B_{Q, f, \ell, C, D}}\langle\alpha \mid y\rangle_{n} .
$$


Démonstration. On commence par traiter le cas où $D=\mathbb{R}^{n}$. Alors $D^{\star}=\{0\}$ et $B_{Q, f, \ell, C, D}=A_{Q, f, \ell} \cap C^{\star}$. La démonstration suit les mêmes idées que celle du théorème 2.1. On commence par montrer que la fonction $b_{Q, f, \ell, C, D}$ est concave. Par le théorème de Hahn Banach, elle s'écrit donc comme la borne inférieure des fonctions affines qui la majorent. Or, la fonction affine $\mathbb{R}^{n} \rightarrow \mathbb{R},\left(y_{1}, \ldots, y_{n}\right) \mapsto \alpha_{1} y_{1}+\cdots+\alpha_{n} y_{n}+\beta$ $\left(\alpha_{i}, \beta \in \mathbb{R}\right)$ majore $b_{Q, f, \ell, C, D}$ si, et seulement si

$$
\forall x \in Q, \forall c \in C, \quad\left\langle x \mid \vec{\ell}-\left(\alpha_{1} \vec{f}_{1}+\cdots+\alpha_{n} \vec{f}_{n}\right)\right\rangle_{E} \leqslant \beta+\langle\alpha \mid c\rangle_{n} .
$$

Si $\alpha \in C^{\star}$, le produit scalaire $\langle\alpha \mid c\rangle_{n}$ est par définition toujours positif ou nul. La valeur minimale qu'il prend lorsque $c$ décrit $C$ est donc 0 . Ainsi, la condition précédente est équivalente à celle qui apparaissait dans la démonstration du théorème 2.1, soit encore à $\beta \geqslant 0$ et $\alpha \in A_{Q, f, \ell}$. Si, au contraire, $\alpha \notin C^{\star}$, alors il existe un vecteur $c_{0} \in C$ tel que $\left\langle\alpha \mid c_{0}\right\rangle_{n}<0$. Comme $C$ est supposé stable par multiplication par les éléments de $\mathbb{R}^{+}$, la quantité $\langle\alpha \mid c\rangle_{n}$ est non minorée lorsque $c$ décrit $C$ et la condition (2.2) n'est jamais satisfaite dans ce cas. La proposition, dans le cas particulier $D=\mathbb{R}^{n}$, résulte de ces considérations.

On en vient maintenant au cas général. D'après ce que l'on vient de faire, il suffit d'établir que :

$$
\begin{aligned}
\inf _{\alpha \in B_{Q, f, \ell, C, D}}\langle\alpha \mid y\rangle_{n} & =\inf _{\alpha \in A_{Q, f, \ell} \cap C^{\star}}\langle\alpha \mid y\rangle_{n} & & \text { si } y \in D \\
& =-\infty & & \text { sinon. }
\end{aligned}
$$

On suppose d'abord que $y \in D$. Alors, si $\alpha$ est un élément de $B_{Q, f, \ell, C, D}$, il s'écrit $\alpha=\alpha_{1}+\alpha_{2}$ avec $\alpha_{1} \in A_{Q, f, \ell} \cap C^{\star}$ et $\alpha_{2} \in D^{\star}$, d'où il suit $\langle\alpha \mid y\rangle_{n}=\left\langle\alpha_{1} \mid y\right\rangle_{n}+\left\langle\alpha_{2} \mid y\right\rangle_{n} \geqslant\left\langle\alpha_{1} \mid y\right\rangle_{n}$. En passant à la borne inférieure, on obtient l'inégalité $\inf _{\alpha \in B_{Q, f, \ell, C, D}}\langle\alpha \mid y\rangle_{n} \geqslant \inf _{\alpha \in A_{Q, f, \ell} \cap C^{\star}}\langle\alpha \mid y\rangle_{n}$. Mais l'inégalité dans l'autre sens est évidente puisque $B_{Q, f, \ell, C, D}$ contient $A_{Q, f, \ell} \cap C^{\star}$. Si maintenant $y \notin D$, le théorème d'Hahn Banach assure qu'il existe $z \in D^{\star}$ tel que $\langle z \mid y\rangle_{n}<0$. Les vecteurs $\lambda z$, pour $\lambda \in \mathbb{R}^{+}$appartiennent alors tous à $B_{Q, f, \ell, C, D}$, ce qui assure que la quantité $\langle\alpha \mid y\rangle_{n}$ est non minorée lorsque $\alpha$ parcourt cet ensemble. On a donc bien démontré ce que l'on voulait dans tous les cas.

Un cas important est celui où le cône convexe $Q$ est défini comme l'intersection d'un nombre fini de demi-espaces, ce qui est la situation que l'on considèrera dans la suite. L'ensemble $B_{Q, f, \ell, C, D}$ est alors un polytope (éventuellement non borné) qui n'a, en tout cas, qu'un nombre fini de sommets. En outre, si on note $\alpha_{1}, \ldots, \alpha_{N}$ ceux qui restent à distance finie, il découle de la proposition précédente que :

$$
\begin{array}{rlll}
b_{Q, f, \ell, C, D}(y) & =\inf _{1 \leqslant i \leqslant N}\left\langle\alpha_{i} \mid y\right\rangle_{n} & & \text { si } y \in D \cap(f(Q)+C) \\
& =-\infty & & \text { sinon. }
\end{array}
$$

Ainsi, déterminer la fonction $b_{Q, f, \ell, C, D}$ revient à déterminer les $\alpha_{i}$.

\section{Un peu de réseaux pour pimenter}

On conserve les notations introduites précédemment, et on se donne en outre $R$ un réseau de $E$, c'est-àdire un sous-groupe additif de $E$ engendré par une base de $E$. On définit une nouvelle fonction $b_{Q, R, f, \ell, C, D}^{\prime}$ : $\mathbb{R}^{n} \rightarrow \mathbb{R}$ par

$$
\begin{aligned}
b_{Q, R, f, \ell, C, D}^{\prime}(y) & =\sup _{x \in Q \cap R, f(x) \in y-C} \ell(x) & & \text { si } y \in D \\
& =-\infty & & \text { sinon. }
\end{aligned}
$$

De même que précédemment, lorsque $D=\mathbb{R}^{n}$, on l'omettra dans la notation. Il est évident que la fonction $b_{Q, R, f, \ell, C, D}^{\prime}$ est majorée par $b_{Q, f, \ell, C, D}$ puisque la borne supérieure pour définir cette dernière fonction est prise sur un ensemble plus gros.

Proposition 2.3. On suppose que $Q$ engendre $E$ en tant qu'espace vectoriel, que l'application $f$ est surjective, et finalement que $R \cap f^{-1}(C)$ et $f^{-1}(C)$ engendrent le même espace vectoriel dans $E$.

Alors, il existe un vecteur $y_{0} \in \mathbb{R}^{n}$ et une constante $c \in \mathbb{R}$ tels que, pour tout $y \in f(R)+C$, on ait :

$$
b_{Q, f, \ell, C}\left(y-y_{0}\right)-c \leqslant b_{Q, R, f, \ell, C}^{\prime}(y) \leqslant b_{Q, f, \ell, C}(y)
$$

où, bien sûr, on convient que $-\infty-c=-\infty$ et $+\infty-c=+\infty$. 
Remarque 2.4. Le fait que $Q$ engendre $E$ n'est évidemment pas vraiment contraignant puisque, dans le cas où cela ne serait pas vérifié, il suffit de remplacer $E$ par le sous-espace vectoriel $Q_{\mathbb{R}}$ engendré par $Q$ et le réseau $R$ par $R \cap Q_{\mathbb{R}}$. On attire toutefois l'attention du lecteur sur le fait qu'il se peut que $R \cap Q_{\mathbb{R}}$ ne soit pas un réseau de $Q_{\mathbb{R}}$; il s'agit donc d'une question qu'il ne faudra pas oublier de se poser le cas échéant. Toutefois, dans le cas où le réseau $R$ et le cône $Q$ sont tous les deux définis sur le corps des nombres rationnels, il est facile de vérifier que $R \cap Q_{\mathbb{R}}$ est toujours un réseau dans $R$; il n'y a donc dans cette situation particulière pas de vérification supplémentaire à faire. De la même façon, la troisième hypothèse de la proposition (à savoir que $R \cap f^{-1}(C)$ et $f^{-1}(C)$ engendrent le même espace vectoriel dans $E$ ) est automatiquement satisfaite dès que $R, C$ et $f$ sont définis sur $\mathbb{Q}$. Dans les applications à suivre, ce sera toujours le cas, et il ne sera donc pas nécessaire de vérifier la troisième hypothèse, de même que l'on pourra appliquer la proposition même si $Q$ n'engendre pas $E$.

Un mot enfin en rapport avec l'hypothèse de surjectivité de $f$. Bien entendu, elle n'est pas véritablement contraignante car on peut toujours appliquer la proposition en remplaçant $\mathbb{R}^{n}$ par l'image de $f$. La conclusion du théorème n'est alors bien sûr plus valable que pout les $y$ qui appartiennent à l'intersection de $f(R)+C$ avec l'image de $f$.

Démonstration. On définit $C^{\prime}=f^{-1}(C)$ et de façon générale, si $X$ est un sous-ensemble de $E$ ou de $\mathbb{R}^{n}$, on note $X_{\mathbb{R}}$ le sous-espace vectoriel qu'il engendre. Soit $M \subset C_{\mathbb{R}}^{\prime}$ une maille du réseau $C_{\mathbb{R}}^{\prime} \cap R$. C'est un ensemble compact qui vérifie la propriété suivante : pour tout $x \in C_{\mathbb{R}}^{\prime}$, il existe $m \in M$ tel que $x+m \in R$. Du fait que $C^{\prime}$ est d'intérieur non vide dans $C_{\mathbb{R}}^{\prime}$ et qu'il est stable par multiplication par les réels positifs, on déduit qu'il existe un translaté de $M$ entièrement inclus dans $\left(-C^{\prime}\right)$. Soit $K$ un tel translaté. C'est encore un ensemble compact qui vérifie une propriété analogue à celle satisfaite par $M$. De même que précédemment, étant donné que $K$ est compact et que $Q$ est un cône convexe d'intérieur non vide dans $Q_{\mathbb{R}}$, il existe $x_{0} \in Q_{\mathbb{R}}$ tel que $x_{0}+K \subset Q$. On définit $y_{0}=f\left(x_{0}\right)$.

Soit $y \in f(R)+C$. Si $y-y_{0} \notin f(Q)+C$, on a $b_{Q, f, \ell, C}\left(y-y_{0}\right)=-\infty$ et la proposition est évidente dans ce cas. On suppose donc que $y-y_{0} \in f(Q)+C$. Alors $b_{Q, f, \ell, C}\left(y-y_{0}\right)$ est fini, et pour tout $\varepsilon>0$, il existe $x_{1} \in Q$ tel que $f\left(x_{1}\right) \in\left(y-y_{0}\right)-C$ et $\ell\left(x_{1}\right) \geqslant b_{Q, f, \ell, C}\left(y-y_{0}\right)-\varepsilon$. On a alors $f\left(x_{0}+x_{1}\right) \in y-C \subset f(R)+C_{\mathbb{R}}$, d'où on déduit que $x_{0}+x_{1} \in R+C_{\mathbb{R}}^{\prime}$ et, de là, qu'il existe $x_{2} \in K$ tel que $x=x_{0}+x_{1}+x_{2}$ soit élément de $R$. Comme $x_{1} \in Q$ et $x_{0}+K \subset Q$, l'élément $x$ appartient aussi à $Q$. Par ailleurs, $f(x)=y_{0}+f\left(x_{1}\right)+f\left(x_{2}\right) \in y_{0}+\left(y-y_{0}\right)-C+f(K)=y-C$ car $f(K)$ est inclus dans $(-C)$ par construction de $K$. Ainsi, trouve-t-on :

$$
b_{Q, R, f, \ell, C}^{\prime}(y) \geqslant \ell(x) \geqslant \ell\left(x_{0}\right)+b_{Q, f, \ell, C}\left(y-y_{0}\right)-\varepsilon \inf _{x^{\prime} \in K} \ell\left(x^{\prime}\right)
$$

et la borne inférieure est finie étant donné que $K$ est compact.

La minoration dans la proposition précédente fait intervenir la valeur de la fonction $b_{Q, f, \ell, C}$ en $y-y_{0}$, alors qu'il aurait été sans doute plus agréable d'avoir simplement $b_{Q, f, \ell, C}(y)$. En général, malheureusement, on ne peut pas remplacer $y-y_{0}$ par $y$, même en modifiant la constante $c$. Néanmoins dans le cas où $Q$ est défini comme l'intersection d'un nombre fini d'hyperplans, on peut être plus précis : il résulte alors de la formule (2.3) que la fonction $b_{Q, f, \ell, C}$ est uniformément continue sur l'ensemble $f(Q)+C$ et donc, en particulier, qu'il existe une constante réelle $c^{\prime}$ telle que :

$$
b_{Q, f, \ell, C}(y)-c^{\prime} \leqslant b_{Q, f, \ell, C}\left(y-y_{0}\right)
$$

pour tout $y \in(f(Q)+C) \cap\left(y_{0}+f(Q)+C\right)$. En fait, l'inégalité précédente estencore satisfaite si $y \notin$ $f(Q)+C$ puisque dans ce cas, le minorant vaut $-\infty$. Enfin, en posant $c^{\prime \prime}=c+c^{\prime}$, il vient :

$$
b_{Q, f, \ell, C, D}(y)-c^{\prime \prime} \leqslant b_{Q, R, f, \ell, C, D}^{\prime}(y) \leqslant b_{Q, f, \ell, C, D}(y)
$$

pour tout $y$ sauf éventuellement ceux qui appartiennent à $D \cap(f(Q)+C)$ mais pas à $y_{0}+f(Q)+C$. Il existe donc, si l'on veut, une zone de trouble autour de la frontière de $f(Q)+C$ sur laquelle on ne sait pas contrôler le comportement de la fonction $b_{Q, R, f, \ell, C, D}^{\prime}$.

\section{2 Étude du cône convexe $\Phi$}

On reprend la situation du théorème 1.10 : on note $I$ l'ensemble des couples d'entiers $(i, j)$ tels que $1 \leqslant i \leqslant j \leqslant d$, et $K$ le sous-ensemble convexe de $\left(\mathbb{R}^{2}\right)^{I}$ défini par les égalités $(1.5)$ et $[1.6)$ et les 
inégalités (1.12). En fait, comme cela a déjà été dit, la formule (1.7) permet de se passer des $\mu_{i, j}$ ce que nous allons faire à partir de maintenant. On considère donc plutôt l'espace vectoriel $E=\mathbb{R}^{I}$ formé des suites $q=\left(q_{i, j}\right)_{(i, j) \in I}$ indexées par les éléments de $I$, et à l'intérieur de celui-ci, le cône convexe $Q$ défini par les jeux d'inégalités suivants :

$$
\begin{aligned}
(\text { Jeu I) }: & \text { si } 1 \leqslant i \leqslant j<d, q_{i, j} \geqslant q_{i, j+1} \\
\text { (Jeu II) }: & \text { si } 1 \leqslant i \leqslant j<d, q_{i, j} \leqslant q_{i+1, j+1} \\
\text { (Jeu III) : } & \text { si } 1 \leqslant i \leqslant j<d, \\
& b q_{j, j}-q_{j, d}+b \cdot \sum_{s=i}^{j-1}\left(q_{s, j}-q_{s, j-1}\right) \geqslant b q_{j+1, j+1}-q_{j+1, d}+b \cdot \sum_{s=i+1}^{j}\left(q_{s, j+1}-q_{s, j}\right)
\end{aligned}
$$

qui correspondent aux inégalités (1.12) dans lesquelles on a remplacé chaque apparition d'un $\mu_{i, j}$ par son expression en fonction des $q_{i, j}$. Le théorème 1.10 dit alors que l'application $\varphi \mapsto\left(q_{i, j}(\varphi)\right)_{(i, j) \in I}$ définit une bijection entre $\Phi$ et $Q$.

On munit $E$ du produit scalaire usuel : si $v=\left(v_{i, j}\right)$ et $w=\left(w_{i, j}\right)$ sont deux éléments de $E$, on pose

$$
\langle v \mid w\rangle_{E}=\sum_{(i, j) \in I} v_{i, j} w_{i, j}
$$

Les inégalités définissant $Q$ se réécrivent alors sous la forme $\left\langle\vec{v}_{m} \mid q\right\rangle_{E} \geqslant 0$ pour certains vecteurs explicites $\vec{v}_{m} \in E(1 \leqslant m \leqslant M)$. Une étude attentive des formules montre en outre que tous les vecteurs $\vec{v}_{m}$ appartiennent à l'hyperplan $\ll$ somme des coordonnées égale $0 \gg$.

On note comme précédemment $Q^{\star}$ le cône dual de $Q$ (par rapport au produit scalaire $\langle\cdot \mid \cdot\rangle_{E}$ ); c'est simplement le cône convexe engendré par les vecteurs $\vec{v}_{m}$. En particulier, il est lui aussi inclus dans l'hyperplan «somme des coordonnées égale $0 \gg$. Malheureusement, la présentation à l'aide des $\vec{v}_{m}$ n'est pas adaptée au calcul des convexes $A_{Q, f, \ell}$ qui apparaissent en 2.1, et qui joueront un rôle central dans la suite de l'article. Pour calculer ces ensembles, il serait plus commode de disposer d'une présentation de $Q^{\star}$ comme l'intersection d'un certain nombre de demi-espaces. Hélas, de part sa complexité, le jeu III d'inégalités rend la chose difficile à réaliser. Pour contourner le problème, l'idée consiste à travailler avec certaines approximations $Q$. Les deux plus simples d'entre elles sont les cônes convexes $Q_{\min }$ et $Q_{\max }$ définis comme suit : $Q_{\max }$ est le cône convexe défini par les jeux d'inégalité I et II, tandis $Q_{\min }$ est celui défini par le jeu I et le jeu II' que voici :

(Jeu II') : $\quad$ si $1 \leqslant i \leqslant j<d, q_{i, j}=q_{i+1, j+1}$

Il est clair que $Q \subset Q_{\max }$ et un calcul aisé montre que $Q_{\min } \subset Q$. Ainsi on a des inclusions renversées au niveau des duaux, d'où on déduit que, pour toute donnée $(f, \ell, C, D)$, l'encadrement

$$
b_{Q_{\min }, f, \ell, C, D} \leqslant b_{Q, f, \ell, C, D} \leqslant b_{Q_{\max }, f, \ell, C, D}
$$

est vrai. Il est maintenant temps de donner les présentations annoncées des cônes duaux $Q_{\min }^{\star}$ et $Q_{\max }^{\star}$. Pour cela, on introduit la définition suivante.

Définition 2.5. Une partie $J$ de $I$ est dite admissible si pour tout couple $(i, j) \in J$, les deux couples $(i, j+1)$ et $(i-1, j-1)$ sont dans $J$, dès qu'ils appartiennent à $I$.

On peut remarquer que les parties admissibles dans le sens précédent sont naturellement en bijection avec les parties de $\{1, \ldots, d\}$ : à une telle partie $J$, on fait correspondre l'ensemble $T$ des nombres non nuls qui sont de la forme :

$$
\operatorname{Card}\{j \geqslant i \mid(i, j) \in J\}
$$

pour un $i \in\{1, \ldots, d\}$. Réciproquement, si $T$ est un sous-ensemble de $\{1, \ldots, d\}$, on note $t_{1}>\cdots>$ $t_{\text {Card } T}$ ses éléments et on lui associe l'ensemble $J \subset I$ formé des couples $(i, j)$ tels que $i \leqslant \operatorname{Card} T$ et $j>d-t_{i}$. On vérifie que les deux applications précédentes sont des bijections inverses l'une de l'autre entre l'ensemble des parties admissibles de $I$ et l'ensemble des parties de $\{1, \ldots, d\}$. Par exemple, si $s \in$ $\{1, \ldots, d\}$, la partie admissible correspondant à $\{1, \ldots, s\}$ est $I_{s}=\{(i, j) \in I \mid j-i \geqslant d-s\}$. 
Proposition 2.6. Une suite $v=\left(v_{i, j}\right)$ de E appartient à $Q_{\min }^{\star}$ si, et seulement si :

$$
\sum_{(i, j) \in I} v_{i, j}=0 \quad \text { et } \forall s \in\{1, \ldots, d\}, \sum_{(i, j) \in I_{s}} v_{i, j} \leqslant 0 .
$$

Une suite $v=\left(v_{i, j}\right)$ de E appartient à $Q_{\max }^{\star}$ si, et seulement si :

$$
\sum_{(i, j) \in I} v_{i, j}=0 \quad \text { et } \quad \forall J \subset I \text { admissible, } \sum_{(i, j) \in J} v_{i, j} \leqslant 0 .
$$

En utilisant l'identification décrite précédemment entre parties admissibles de $I$ et parties de $\{1, \ldots, d\}$, on peut réécrire la condition d'appartenant à $Q_{\max }^{\star}$ comme suit :

$$
\sum_{(i, j) \in I} v_{i, j}=0 \quad \text { et } \forall T \subset\{1, \ldots, d\}, \sum_{s=1}^{\operatorname{Card} T} \sum_{j=s+1}^{t_{s}} v_{j-s, j-1} \leqslant 0
$$

où $t_{s}$ est le $s$-ième plus petit élément de $T$.

\section{Démonstration de la proposition 2.6: un peu de théorie des flots}

Il est possible de donner une démonstration « à la main » de la proposition 2.6 mais, comme me l'a signalé Bodo Lass, la proposition peut également se déduire du théorème Flot-Maximal-Coupe-Minimale, classique en théorie des graphes. J'ai choisi ici de présenter cette dernière approche qui est à la fois plus générale et plus conceptuelle.

Quelques rappels pour commencer au sujet du théorème Flot-Maximal-Coupe-Maximale. Soit $G$ un graphe fini orienté dans lequel on a privilégié deux sommets $D$ (comme départ) et $A$ (comme arrivée) et on a attribué à chaque arête $a$ un nombre positif ou nul, éventuellement égal à $+\infty$, appelé capacité de $a$, et noté $c(a)$. Si $a$ est une arête dans $G$, on note $s_{1}(a)$ (resp. $s_{2}(a)$ ) le sommet duquel elle part (resp. auquel elle aboutit). Un flot de $D$ vers $A$ est une fonction $f$ à valeurs réelles définie sur les arêtes de $G$ satisfaisant les propriétés suivantes :

- pour toute arête $a$, on a $0 \leqslant f(a) \leqslant c(a)$;

- pour tout sommet $s$ différent de $D$ et $A$, on a $\sum_{a \mid s_{1}(a)=s} f(a)=\sum_{a \mid s_{2}(a)=s} f(a)$. La dernière propriété implique que :

$$
\sum_{a \mid s_{1}(a)=D} f(a)-\sum_{a \mid s_{2}(a)=D} f(a)=\sum_{a \mid s_{2}(a)=A} f(a)-\sum_{a \mid s_{1}(a)=A} f(a) .
$$

Cette valeur commune s'appelle la valeur du flot $f$ et est notée $|f|$. Une coupe $C$ de $G$ est la donnée d'une partition de l'ensemble des sommets de $G$ en deux parties $\mathcal{D}$ et $\mathcal{A}$ telles que $D \in \mathcal{D}$ et $A \in \mathcal{A}$. La capacité de la coupe $C$, que l'on note $|C|$, est la somme des $c(a)$ étendue à toutes les arêtes $a$ qui ont leur origine dans $\mathcal{D}$ et leur arrivée dans $\mathcal{A}$.

Il est facile de voir que si $f$ est un flot et $C$ est une coupe sur le graphe $G$ précédemment fixé, alors $|f| \leqslant|C|$. Ainsi, en passant aux bornes supérieures et inférieures, on obtient $\sup _{f \text { flot }}|f| \leqslant \min _{C}$ coupe $|C|$ (notez qu'il n'y a qu'un nombre fini de coupes possibles).

Théorème 2.7 (Flot-Maximal-Coupe-Minimale). Avec les notations précédentes, on a :

$$
\sup _{f \text { flot }}|f|=\min _{C \text { coupe }}|C|
$$

et la borne supérieure précédente est atteinte.

Démonstration. Voir par exemple [13].

On se place à présent dans une situation un peu différente. On considère toujours un graphe fini orienté $G$ mais on ne se donne plus de décoration : on ne suppose plus que deux de ses sommets sont privilégiés, ni que les arêtes de $G$ sont munies d'une capacité. On note $S$ l'ensemble des sommets de $G$. À un tel graphe, on associe l'espace euclidien $E_{G}=\mathbb{R}^{S}$ muni du produit scalaire usuel $\langle\cdot \mid \cdot\rangle_{G}$ et $Q_{G}$ le cône convexe regroupant les éléments $x=\left(x_{s}\right)_{s \in S} \in E_{G}$ vérifiant

$$
x_{s_{2}(a)} \leqslant x_{s_{1}(a)}
$$

pour toute arête $a$ de $G$. 
Définition 2.8. Un sous-ensemble $S^{\prime}$ de $S$ est dit admissible si toute arête de $G$ ayant son origine dans $S^{\prime}$ a aussi son but dans $S^{\prime}$.

On a alors la proposition suivante, de laquelle il résulte facilement la proposition 2.6.

Proposition 2.9. On conserve les notations précédentes, et on note $Q_{G}^{\star}$ le cône dual de $Q_{G}$ par rapport au produit scalaire $\langle\cdot \mid \cdot\rangle_{G}$. Alors un élément $x=\left(x_{s}\right)_{s \in S} \in E_{G}$ appartient à $Q_{G}^{\star}$ si, et seulement si :

$$
\sum_{s \in S} x_{s}=0 \quad \text { et } \quad \forall S^{\prime} \subset S \text { admissible, } \sum_{s \in S^{\prime}} x_{s} \leqslant 0 .
$$

Démonstration. De la définition de $Q_{G}$, il résulte que $Q_{G}^{\star}$ est un cône convexe engendré par des vecteurs de l'hyperplan « somme des coordonnées égale $0 \gg$. Ainsi $Q_{G}^{\star}$ est inclus dans cet hyperplan. Par ailleurs, si $S^{\prime} \subset S$ est un ensemble admissible de sommets, l'opposé de la fonction indicatrice de $S^{\prime}$ définit un vecteur $-\mathbb{1}_{S^{\prime}} \in Q_{G}$. On en déduit que le produit scalaire $\left\langle x \mid \mathbb{1}_{S^{\prime}}\right\rangle_{G}$ est négatif ou nul dès que $x \in Q_{G}^{\star}$. Les éléments de $Q_{G}^{\star}$ vérifient donc bien les conditions de la proposition.

Réciproquement, on considère un vecteur $x=\left(x_{s}\right) \in E_{G}$ vérifiant ces conditions. Soit $M$ un nombre réel positif assez grand pour que toutes les sommes $x_{s}+M$ (pour $s$ décrivant $S$ ) soient positives ou nulles. On introduit le graphe $\tilde{G}$ obtenu à ajoutant à $G$ deux nouveaux sommets notés $D$ et $A$ et, pour tout sommet $s$ de $G$, une arête de $D$ vers $s$ et une arête de $s$ vers $A$. On définit une capacité $c$ sur $\tilde{G}$ comme suit :

- si $a$ est une arête de $G$, on pose $c(a)=+\infty$;

- si $a$ part de $D$ et arrive à un sommet $s$ de $G$, on pose $c(a)=x_{s}+M$;

- si $a$ part d'un sommet $s$ de $G$ et arrive à $A$, on pose $c(a)=M$.

Soit $C=(\mathcal{D}, \mathcal{A})$ une coupe de $\tilde{G}$. L'ensemble $S^{\prime}=\mathcal{D} \backslash\{D\}$ est inclus dans $S$. Si $S^{\prime}$ n'est pas admissible, cela signifie qu'il existe une arête reliant un sommet de $S^{\prime}$ à un sommet de $S \backslash S^{\prime}$. Autrement dit, il existe une arête dans $G$ (donc de capacité infinie) reliant un sommet de $\mathcal{D}$ à un sommet de $\mathcal{A}$. Dans ce cas, la capacité de la coupe $C$ est donc infinie. $\mathrm{Si}$, au contraire, l'ensemble des sommets $S^{\prime}$ est admissible, la capacité de la coupe $C$ vérifie :

$$
|C|=\sum_{s \in S^{\prime}} M+\sum_{s \in S \backslash S^{\prime}}\left(x_{s}+M\right)=n M-\sum_{s \in S^{\prime}} x_{s} \geqslant n M
$$

où on a noté $n$ le nombre de sommets de $G$. Ainsi la capacité minimale d'une coupe, notée $c_{\min }$ est, elle aussi, supérieure ou égale à $n M$. D'après le théorème Flot-Maximal-Coupe-Minimale, il existe un flot $f$ sur $\tilde{G}$, de valeur $c_{\min }$. Comme la somme des capacités des arêtes sortant de $D$ est $n M$, la capacité de ce flot est inférieure ou égale à $n M$. On en déduit qu'elle est égale à $n M$ (et donc qu'il en est de même de $\left.c_{\min }\right)$ et que toute arête partant de $D$ est saturée, c'est-à-dire que pour toute arête $a$ partant de $D$, on a $f(a)=c(a)=M+x_{s_{2}(a)}$. De même, on démontre que, pour toute arête $a$ partant d'un sommet $s \in S$ et aboutissant en $A$, on a $f(a)=c(a)=M$. Par la condition de flot, on a pour tout sommet $s \in S$ :

$$
\sum_{a \mid s_{1}(a)=s} f(a)-\sum_{a \mid s_{2}(a)=s} f(a)=x_{s}
$$

où, dans les sommes précédentes, $a$ désigne une arête de $G$. Ainsi, pour tout $y=\left(y_{s}\right) \in Q_{G}$, on a :

$$
\langle x \mid y\rangle_{E}=\sum_{s \in S} y_{s} \cdot\left(\sum_{a \mid s_{1}(a)=s} f(a)-\sum_{a \mid s_{2}(a)=s} f(a)\right)=\sum_{a}\left(y_{s_{1}(a)}-y_{s_{2}(a)}\right) f(a) \geqslant 0
$$

d'où on déduit finalement que $x \in Q_{G}^{\star}$ comme voulu.

\subsection{Un premier exemple d'application}

À titre d'exemple, et afin de familiariser le lecteur avec les méthodes de cet article, j'aimerais montrer comment la machinerie qui vient d'être introduite permet de démontrer la majoration du théorème 1 g sous l'hypothèse supplémentaire $b \geqslant d-1$. Ce cas est intéressant car de nombreuses difficultés techniques s'évanouïssent, mais il permet tout de même de donner une idée correcte de la nature des raisonnements qui apparaîtront dans la section suivante.

Pour ne pas avoir, dans la suite, à distinguer systématiquement les cas selon que $h$ soit ou non égal à 0 , on se restreint à partir de maintenant à $h \neq 0$, le cas contraire se traitant de façon complètement analogue en ajoutant $\frac{d(d-1)}{2}$ à tous les majorants. 


\subsubsection{Positionnement du problème}

Un réseau $L$ de $M$ définit un $k$-point de la variété $\mathcal{X}_{\leqslant e}$ si, et seulement si les exposants des diviseurs élémentaires du $k[[u]]$-module engendré par $\sigma(L)$ par rapport à $L$ sont tous compris entre 0 et $e$. Si on note ceux-ci $\mu_{1}(L) \geqslant \cdots \geqslant \mu_{d}(L)$ comme dans la proposition 1.2, cela se réécrit $\mu_{1}(L) \leqslant e$ et $\mu_{d}(L) \geqslant$ 0 . Par ailleurs, si on note $q_{i, j}(L)$ et $\mu_{i, j}(L)$ les nombres réels associés au $d$-uplet de fonctions $\varphi(L)=$ $\left(\varphi_{1}(L), \varphi_{2}(L), \ldots, \varphi_{d}(L)\right)$, l'assertion 4 de la proposition 1.2 combinée à la proposition 1.7 assure que $\mu_{j}(L)=\mu_{1, j}(L)$ pour tout indice $j \in\{1, \ldots, d\}$. Ces observations conduisent à une décomposition de la variété $\mathcal{X}_{\leqslant e}$ en union disjointe de parties localement fermées comme suit :

$$
\mathcal{X}_{\leqslant e}=\bigsqcup_{\varphi \in \Phi \leqslant e} \mathcal{X}_{\varphi}
$$

où l'ensemble $\Phi_{\leqslant e}$ réunit les $\varphi \in \Phi_{\mathbb{Z}}$ tels que $\mu_{1,1}(\varphi) \leqslant e$ et $\mu_{1, d}(\varphi) \geqslant 0$. On déduit directement de cette écriture, une formule au niveau des dimensions :

$$
\operatorname{dim}_{k} \mathcal{X}_{\leqslant e}=\sup _{\varphi \in \Phi \leqslant e} \operatorname{dim}_{k} \mathcal{X}_{\varphi}
$$

Comme précédemment, on note $E$ l'espace des suites indicées par l'ensemble $I$, et $Q$ le cône convexe défini par les jeux d'inégalités I, II et III. Soit $f: E \rightarrow \mathbb{R}^{2}$ l'application linéaire qui à une famille $\left(q_{i, j}\right)$ associe le couple $\left(\mu_{1,1}, \mu_{1, d}\right)$ où ces réels sont définis comme d'habitude par la formule (1.7). Par ailleurs, par le lemme 1.15, la fonction dim s'étend en une forme linéaire $E \rightarrow \mathbb{R}$, notée $\ell$. On pose encore $C=$ $\mathbb{R}^{+} \times \mathbb{R}^{-}, D=\mathbb{R}^{2}$ et on note $R$ le réseau formé des éléments $\left(q_{i, j}\right) \in E$ tels que $q_{i, j} \in \frac{1}{b} \mathbb{Z}$ pour tout $(i, j) \in I$ et $q_{i, d} \in \mathbb{Z}$ pour tout $i \in\{1, \ldots, d\}$. Les théorèmes 1.10 et 1.18 , couplés à la proposition 1.11 , fournissent alors l'égalité (on rappelle que l'on suppose $h \neq 0$ ) :

$$
\operatorname{dim}_{k} \mathcal{X}_{\leqslant e}=b_{Q, R, f, \ell, C}^{\prime}(e, 0)
$$

et donc en particulier $\operatorname{dim}_{k} \mathcal{X}_{\leqslant e} \leqslant b_{Q, R, f, \ell, C}(e, 0)$. Pour estimer ce dernier nombre, on utilise l'encadrement (2.5), et les propositions 2.2 et 2.6 pour évaluer les fonctions $b_{Q_{\min }, f, \ell, C}$ et $b_{Q_{\max }, f, \ell, C}$.

\subsubsection{Les vecteurs $\vec{\mu}_{1}, \ldots, \vec{\mu}_{d}$ et $\vec{\delta}$}

Pour pouvoir suivre la méthode indiquée ci-dessus, il faut commencer par exprimer les coordonnées de la fonction $f$ ainsi que la forme linéaire $\ell$ comme des produits scalaires contre certains vecteurs de $E$. C'est l'objet de ce numéro.

Pour un indice $i$ compris entre 1 et $d$, on appelle $\vec{\mu}_{i}$ le vecteur de $E$ tel que pour toute suite $q=\left(q_{i, j}\right) \in$

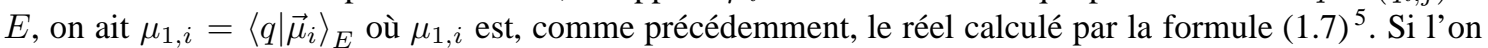
décide de représenter les éléments de $E$ comme des matrices triangulaires supérieures (le terme d'indice $(i, j)$ étant placé à l'intersection de la $i$-ième ligne et de la $j$-ième colonne), on a ainsi :

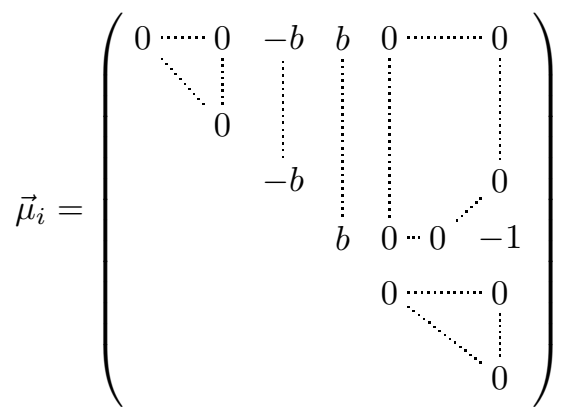

où le $b$ sur la diagonale est à l'intersection de la $i$-ième ligne et de la $i$-ième colonne. De même, on définit

\footnotetext{
5. Pour le calcul de la dimension de $\mathcal{X}_{\leqslant e}$, seuls les vecteurs $\vec{\mu}_{1}$ et $\vec{\mu}_{d}$ seront utiles. Cependant, les autres vecteurs $\vec{\mu}_{i}$ serviront dans la suite pour estimer la dimension d'autres variétés, et nous avons pensé qu'il était préférable de les introduire tous en même temps.
} 
le vecteur $\vec{\delta} \operatorname{par}\langle q \mid \vec{\delta}\rangle_{E}=\ell(q)$ pour tout $q \in E$. Le lemme 1.15 montre que $\vec{\delta}$ s'exprime comme suit :

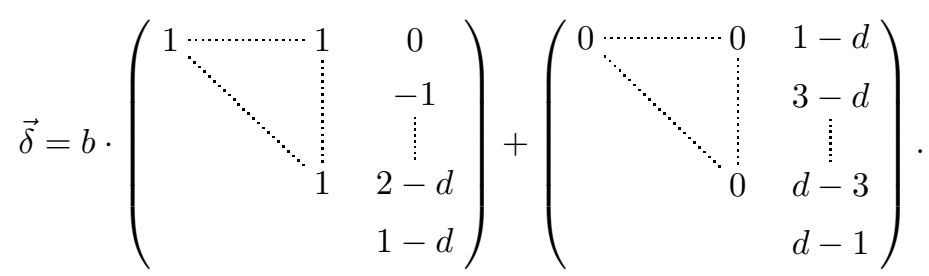

Si $J \subset I$ est un sous-ensemble admissible (voir définition 2.5p et si $x=\left(x_{i, j}\right) \in E$, on note $S_{J}(x)$ la somme des coordonnées $x_{i, j}$ pour $(i, j)$ parcourant $J$. Les fonctions $S_{J}$ ainsi définies sont clairement des formes linéaires sur $E$. Si $T$ est la partie de $\{1, \ldots, d\}$ correspondant à l'ensemble admissible $J$, on s'autorise à noter $S_{T}$ à la place de $S_{J}$. Pour les calculs à suivre, le lemme suivant nous sera fort utile.

Lemme 2.10. Si $T$ est un sous-ensemble de $\{1, \ldots, d\}$, on a :

$$
S_{T}\left(\vec{\mu}_{i}\right)=b \cdot \mathbb{1}_{T}(d+1-i)-[(\operatorname{Card} T) \geqslant i]
$$

où $\mathbb{1}_{T}$ est la fonction indicatrice de $T$ et l'expression $[(\operatorname{Card} T) \geqslant i]$ vaut 1 si $\operatorname{Card} T \geqslant i$ et 0 sinon. On a aussi :

$$
S_{T}(\vec{\delta})=b \cdot\left(\sum_{t \in T} t-\frac{\operatorname{Card} T \cdot(\operatorname{Card} T+1)}{2}\right)-\operatorname{Card} T \cdot(d-\operatorname{Card} T) .
$$

Démonstration. C'est un simple calcul à partir des descriptions précédentes.

\subsubsection{Calcul du majorant}

On commence par calculer la fonction $b_{Q_{\min }, f, \ell, C}$ en utilisant la proposition 2.2. Il s'agit donc de déterminer l'ensemble $B_{Q_{\min }, f, \ell, C}=A_{Q_{\min }, f, \ell} \cap C^{\star}$. Il est facile de voir que $C^{\star}=\mathbb{R}^{+} \times \mathbb{R}^{-}$; reste donc à calculer $A_{Q_{\min }, f, \ell}$. Comme $f=\left(\left\langle\vec{\mu}_{1}, \cdot\right\rangle_{E},\left\langle\vec{\mu}_{d}, \cdot\right\rangle_{E}\right)$ et $\ell=\langle\vec{\delta} \mid \cdot\rangle_{E}$, la formule (2.1) s'écrit :

$$
A_{Q_{\text {min }}, f, \ell}=\left\{\left(y_{1}, y_{d}\right) \in \mathbb{R}^{2} \mid y_{1} \vec{\mu}_{1}+y_{d} \vec{\mu}_{d}-\vec{\delta} \in Q_{\text {min }}^{\star}\right\} .
$$

On cherche donc les couples $\left(y_{1}, y_{d}\right)$ tels que $y_{1} \geqslant 0, y_{d} \leqslant 0$, et $y_{1} \vec{\mu}_{1}+y_{d} \vec{\mu}_{d}-\vec{\delta} \in Q_{\min }^{\star}$. D'après la proposition 2.6, la dernière condition se réécrit :

$$
\left\{\begin{array}{l}
y_{1} S_{I_{s}}\left(\vec{\mu}_{1}\right)+y_{d} S_{I_{s}}\left(\vec{\mu}_{d}\right) \leqslant S_{I_{s}}(\vec{\delta}) \quad \forall s \in\{1, \ldots, d\} \\
y_{1} S_{I_{d}}\left(\vec{\mu}_{1}\right)+y_{d} S_{I_{d}}\left(\vec{\mu}_{d}\right)=S_{I_{d}}(\vec{\delta})
\end{array}\right.
$$

où on rappelle que $I_{s}$ désigne l'ensemble admissible formé des couples $(i, j) \in I$ tels que $j-i \geqslant d-s$. Comme cet ensemble correspond à la partie $T=\{1, \ldots, s\}$, le lemme 2.10 assure que si $s<d$, on a $S_{I_{s}}\left(\vec{\mu}_{1}\right)=-1, S_{I_{s}}\left(\vec{\mu}_{d}\right)=b$ et $S_{I_{s}}(\vec{\delta})=-s(d-s)$, tandis que pour $s=d$, on a $S_{I_{d}}\left(\vec{\mu}_{1}\right)=S_{I_{d}}\left(\vec{\mu}_{d}\right)=b-1$, $S_{I_{d}}(\vec{\delta})=0$. Ainsi, le système (2.8) est équivalent à :

$$
y_{1}+y_{d}=0 \quad \text { et } \quad y_{1} \geqslant \frac{s(d-s)}{b+1}, \quad \forall s \in\{1, \ldots, d-1\} .
$$

Comme on voit aisément que le maximum de $s(d-s)$ vaut $\left[\frac{d^{2}}{4}\right]$, l'ensemble $B_{Q_{\min }, f, \ell, C}$ est formé des couples $(y,-y)$ avec $y \geqslant\left[\frac{d^{2}}{4}\right] \cdot \frac{1}{b+1}$. Ainsi, si $y_{1} \geqslant y_{d}$, on obtient :

$$
b_{Q_{\min }, f, \ell, C}\left(y_{1}, y_{d}\right)=\left[\frac{d^{2}}{4}\right] \cdot \frac{y_{1}-y_{d}}{b+1} .
$$

On souhaite à présent montrer que l'expression ci-dessus vaut encore pour la fonction $b_{Q_{\max }, f, \ell, C}$. Comme on sait que $b_{Q_{\min }, f, \ell, C} \leqslant b_{Q_{\max }, f, \ell, C}$, il suffit, pour établir ce que l'on veut, de montrer que ([ $\left[\frac{d^{2}}{4}\right] \cdot \frac{1}{b+1},-\left[\frac{d^{2}}{4}\right]$. $\left.\frac{1}{b+1}\right) \in B_{Q_{\max }, f, \ell, C}$, c'est-à-dire que pour toute partie $T \in\{1, \ldots, d\}$, on a :

$$
\left[\frac{d^{2}}{4}\right] \cdot\left(S_{T}\left(\vec{\mu}_{1}\right)-S_{T}\left(\vec{\mu}_{d}\right)\right) \leqslant(b+1) \cdot S_{T}(\vec{\delta}) .
$$


(Notez que le cas d'égalité, qui doit être obtenu pour $T=\{1, \ldots, d\}$, a déjà été vérifié.) On commence par éliminer les cas triviaux $T=\emptyset$ et $T=\{1, \ldots, d\}$. Ceci permet d'écrire $S_{T}\left(\vec{\mu}_{1}\right)=b \mathbb{1}_{T}(d)-1$ et $S_{T}\left(\vec{\mu}_{d}\right)=\mathbb{1}_{T}(1)$. Par ailleurs, si $T=\left\{t_{1}, \ldots, t_{s}\right\}$ avec $t_{1}<\cdots<t_{s}$, la formule du lemme 2.10 se réécrit

$$
S_{T}(\vec{\delta})=-s(d-s)+b \sum_{i=1}^{s}\left(t_{i}-i\right)
$$

On distingue à présent quatre cas selon que les entiers 1 et $d$ appartiennent ou n'appariennent pas à $T$. Si $1 \in T$ et $d \notin T$, alors $S_{T}\left(\vec{\mu}_{1}\right)-S_{T}\left(\vec{\mu}_{d}\right)=-b-1$ et l'inégalité à démontrer devient $S_{T}(\vec{\delta}) \geqslant-\left[\frac{d^{2}}{4}\right]$. Or les $t_{i}-i$ étant tous positifs ou nuls, la formule (2.9) montre que $S_{t}(\vec{\delta}) \geqslant-s(d-s)$ d'où il suit ce que l'on veut. Pour les autres cas, il est important de remarquer que si $1 \notin T$, alors tous les $t_{i}-i$ sont supérieurs ou égaux à 1 , alors que si $d \in T$, on a $t_{s}-s=d-s$. En particulier, si l'on excepte le cas que l'on vient de traiter, c'est-à-dire si l'on suppose que $1 \notin T$ ou que $d \in T$, on a

$$
S_{T}(\vec{\delta}) \geqslant b \cdot \min (s, d-s)-s(d-s) \text {. }
$$

Comme on a supposé $b \geqslant d-1$, on en déduit que $S_{T}(\vec{\delta}) \geqslant 0$, ce qui suffit à conclure dans le cas où les deux nombres 1 et $d$ appartiennent (resp. n'appartiennent pas) à $T$ puisqu'alors $S_{T}\left(\vec{\mu}_{1}\right)-S_{T}\left(\vec{\mu}_{d}\right)=-1 \leqslant 0$. Si finalement, $1 \notin T$ et $d \in T$, la minoration de $S_{T}(\delta)$ est renforcée comme suit :

$$
S_{T}(\vec{\delta}) \geqslant b(s-1+d-s)-s(d-s)=b(d-1)-s(d-s) \geqslant b(d-1)-\left[\frac{d^{2}}{4}\right] .
$$

Étant donné que $S_{T}\left(\vec{\mu}_{1}\right)-S_{T}\left(\vec{\mu}_{d}\right)=b-1$ dans ce cas, il suffit d'établir

$$
(b+1)\left(b(d-1)-\left[\frac{d^{2}}{4}\right]\right) \geqslant(b-1) \cdot\left[\frac{d^{2}}{4}\right],
$$

ce qui se simplifie encore en $(b+1)(d-1) \geqslant 2\left[\frac{d^{2}}{4}\right]$. Finalement, la condition $b \geqslant d-1$ assure que cette inégalité est bien satisfaite. On a ainsi démontré que

$$
b_{Q_{\max }, f, \ell, C}\left(y_{1}, y_{d}\right)=\left[\frac{d^{2}}{4}\right] \cdot \frac{y_{1}-y_{d}}{b+1} \quad \text { pour } y_{1} \geqslant y_{d} .
$$

à partir de quoi la majoration du théorème 2 s'ensuit puisque l'on sait que la dimension de $\mathcal{X}_{\leqslant e}$ est majorée $\operatorname{par} b_{Q, f, \ell, C}(e, 0)$ lui même majoré par $b_{Q_{\max }, f, \ell, C}(e, 0)$.

\section{Dimension des variétés $\mathcal{X}_{\leqslant e}, \mathcal{X}_{\mu}$ et $\mathcal{X}_{\leqslant \mu}$}

Pour estimer les dimensions des variérés $\mathcal{X}_{\leqslant e}, \mathcal{X}_{\mu}$ et $\mathcal{X}_{\leqslant \mu}$, on suit la méthode introduite précédemment, sauf que l'on remplace la fonction $f$ par la fonction

$$
g: E \rightarrow \mathbb{R}^{d}, \quad q \mapsto\left(\left\langle\vec{\mu}_{1} \mid q\right\rangle_{E},\left\langle\vec{\mu}_{2} \mid q\right\rangle_{E}, \ldots,\left\langle\vec{\mu}_{d} \mid q\right\rangle_{E}\right)
$$

et que la lettre $C$ fait désormais référence au nouveau cône convexe $\left\{\left(y_{1}, \ldots, y_{d}\right) \in \mathbb{R}^{d} \mid y_{1} \geqslant \cdots \geqslant y_{d}\right\}$ Toutes les autres notations $\left(Q, R, Q_{\min }, Q_{\max }, \ell, \vec{\mu}_{i}, \vec{\delta}\right.$, etc.) sont conservées; on se contente donc de renvoyer le lecteur aux $\S \S 2.2$ et 2.3.2 s'il souhaite se remémorer les définitions. Le cône dual de $C$ jouera un rôle particulier dans la suite; on remarque d'ores et déjà qu'il est engendré par les vecteurs de la forme $(0, \ldots, 0,1,-1,0, \ldots, 0)$. On en déduit facilement une description en termes d'inégalités :

$$
C^{\star}=\left\{\begin{array}{l|l}
\left(z_{1}, \ldots, z_{d}\right) \in \mathbb{R}^{d} & \begin{array}{l}
z_{1}+\cdots+z_{d}=0 \\
z_{1}+\cdots+z_{i} \geqslant 0, \forall i \in\{1, \ldots, d\}
\end{array}
\end{array}\right\} .
$$

D'autre part, étant donné que les vecteurs $\vec{\mu}_{i}$ forment trivialement une famille libre dans $E$, l'application linéaire $g$ est surjective. On peut ainsi appliquer la proposition 2.3 avec les données précédentes.

À partir de maintenant, on se restreint à nouveau au cas où $h \neq 0$, le cas contraire se traitant exactement de la même façon sauf qu'il faut ajouter $\frac{d(d-1)}{2}$ à tous les majorants. Par le théorème 1.18 , pour $\mu=$ $\left(\mu_{1}, \ldots, \mu_{d}\right)$ vérifiant les bonnes hypothèses, la dimension de $\mathcal{X}_{\mu}$ (resp. $\mathcal{X}_{\leqslant \mu}$ ) est majorée (et même bien approchée en vertu de la proposition 2.3) par le nombre $b_{Q, g, \ell, 0}(\mu)$ (resp. $b_{Q, g, \ell, C^{\star}}(\mu)$ ). On est ainsi ramené à calculer $b_{Q, g, \ell, 0}(\mu)$ et $b_{Q, g, \ell, C^{\star}}(\mu)$, et pour cela, à étudier les ensembles $B_{Q, g, \ell, 0}$ et $B_{Q, g, \ell, C^{\star}}$. 


\subsection{Les points extrémaux de $A_{Q_{\max }, g, \ell}$}

Soit $\mathfrak{S}_{d}$ l'ensemble des permutations de l'ensemble $\{1, \ldots, d\}$. Pour tout $w \in \mathfrak{S}_{d}$, on définit le vecteur $\vec{\rho}_{w} \in \mathbb{R}^{d}$ par :

$$
\vec{\rho}_{w}=\left((b-1) \cdot \sum_{n=1}^{\infty} \frac{d+1-i-w^{n}(i)}{b^{n}}\right)_{1 \leqslant i \leqslant d} .
$$

Il est clair que la somme infinie que l'on vient d'écrire converge. En fait, la suite des $w^{n}(i)$ étant périodique, elle converge même vers un nombre rationnel (qui s'exprime comme l'évaluation en $b$ d'une fraction rationnelle à coefficients entiers). De plus, lorsque $w=w_{0}$ est la permutation $i \mapsto d+1-i$, le vecteur $\vec{\rho}_{w_{0}}$ s'exprime facilement : il vaut $\frac{2 \vec{\rho}}{b+1}$ où on rappelle que $\vec{\rho}=\left(\frac{d-1}{2}, \frac{d-3}{2}, \ldots, \frac{1-d}{2}\right)$. Enfin, à partir de maintenant, on pose $b_{0}=1+\left[\frac{(d-1)^{2}}{4}\right]$.

Proposition 3.1. On suppose $b \geqslant b_{0}$. Les points extrémaux de $A_{Q_{\max }, g, \ell}$ sont exactement les vecteurs $\vec{\rho}_{w}$ pour $w$ parcourant $\mathfrak{S}_{d}$.

La fin de cette partie est consacrée à la démonstration de la proposition. On peut d'ores et déjà présenter le convexe $A_{Q_{\max }, g, \ell}$ comme une intersection de demi-espaces; il suffit pour cela d'injecter la description de $Q_{\max }^{\star}$ donnée par la proposition 2.6 dans la formule (2.1.). La condition qui en découle s'exprime simplement à l'aide des fonctions $S_{T}$ introduites juste avant le lemme 2.10; elle dit qu'un élément $y=\left(y_{1}, \ldots, y_{d}\right) \in$ $\mathbb{R}^{d}$ appartient à $A_{Q_{\max }, g, \ell}$ si, et seulement si

$$
y_{1} S_{T}\left(\vec{\mu}_{1}\right)+y_{2} S_{T}\left(\vec{\mu}_{2}\right)+\cdots+y_{d} S_{T}\left(\vec{\mu}_{d}\right) \leqslant S_{T}(\vec{\delta})
$$

pour toute partie $T \subset\{1, \ldots, d\}$ et l'égalité est atteinte lorsque $T=\{1, \ldots, d\}$. Avec les expressions du lemme 2.10, ceci se réécrit :

$$
\left\{\begin{array}{l}
y_{1}+y_{2}+\cdots+y_{d}=0 \\
f_{T}(y) \geqslant 0, \quad \forall T \subsetneq\{1, \ldots, d\}
\end{array}\right.
$$

où on a posé $s=$ Card $T$ et :

$$
f_{T}(y)=\left(y_{1}+\cdots+y_{s}\right)-b \cdot \sum_{t \in T} y_{d+1-t}-s(d-s)+b \cdot\left(\sum_{t \in T} t-\frac{s(s+1)}{2}\right) .
$$

Ceci étant dit, un point extrémal de $A_{Q_{\max }, g, \ell}$ n'est autre qu'un point de $A_{Q_{\max }, g, \ell}$ qui se situe à l'intesection de $d-1$ hyperplans affines indépendants parmi ceux d'équation $f_{T}(x)=0$ avec $T \subsetneq\{1, \ldots, d\}$. Calculer ces points revient donc à déterminer une condition nécessaire et suffisante sur les parties $T_{1}, \ldots, T_{d-1}$ de $\{1, \ldots, d\}$ pour que :

i) en posant $T_{d}=\{1, \ldots, d\}$, les hyperplans affines d'équation $f_{T_{i}}(x)=0(1 \leqslant i \leqslant d)$ aient un unique point d'intersection, et

ii) ce point d'intersection appartienne à $A_{Q_{\max }, g, \ell}$.

On commence par deux lemmes.

Lemme 3.2. On considère des entiers $m$ et $n$ tels que $0 \leqslant m<n \leqslant d$ et un élément $y=\left(y_{1}, \ldots, y_{d}\right) \in$ $A_{Q_{\max }, g, \ell \text {. Alors : }}$

$$
-m(n-m) \leqslant y_{m+1}+y_{m+2}+\cdots+y_{n} \leqslant(n-m)(d-n) .
$$

Démonstration. On commence par prouver la majoration. Dans le cas où $m=0$, celle-ci provient directement de l'inégalité $f_{T}(y) \geqslant 0$ où $T=\{d+1-n, \ldots, d\}$. Dans le cas où $m>0$, on considère la partie $T=\{d+1-n, \ldots, d-m\}$. L'inégalité $f_{T}(y) \geqslant 0$ donne alors :

$$
\left(y_{1}+\cdots+y_{n-m}\right)-b\left(y_{m+1}+\cdots+y_{n}\right) \geqslant(n-m)(d-n+m)-b(n-m)(d-n) .
$$

Or, on sait déjà par ailleurs que $y_{1}+\cdots+y_{n-m} \leqslant(n-m)(d-n+m)$. Le résultat s'ensuit. Pour la minoration, le plus rapide est de remarquer que l'application $\left(y_{1}, \ldots, y_{d}\right) \mapsto\left(-y_{d}, \ldots,-y_{1}\right)$ définit une bijection de $A_{Q_{\max }, g, \ell}$ dans lui-même et que, via cette bijection, la majoration qui vient d'être établie donne la minoration. 


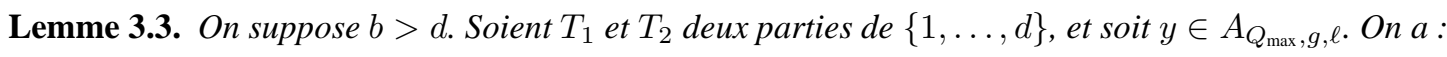

$$
f_{T_{1}}(y)+f_{T_{2}}(y) \geqslant f_{T_{1} \cap T_{2}}(y)+f_{T_{1} \cup T_{2}}(y)
$$

et l'égalité a lieu si, et seulement si $T_{1} \subset T_{2}$ ou $T_{2} \subset T_{1}$.

Démonstration. Soient $s_{1}, s_{2}, s_{1 \cap 2}$ et $s_{1 \cup 2}$ les cardinaux respectifs de $T_{1}, T_{2}, T_{1} \cap T_{2}$ et $T_{1} \cup T_{2}$. On a évidemment $s_{1}+s_{2}=s_{1 \cap 2}+s_{1 \cup 2}$ et $s=s_{1}-s_{1 \cap 2}=s_{1 \cup 2}-s_{2} \geqslant 0$. Par ailleurs, quitte à intervertir $T_{1}$ et $T_{2}$, on peut supposer $s_{1} \leqslant s_{2}$. Un calcul immédiat conduit alors à

$$
\begin{aligned}
f_{T_{1}}(y) & +f_{T_{2}}(y)-f_{T_{1} \cap T_{2}}(y)-f_{T_{1} \cup T_{2}}(y) \\
& =\left(y_{s_{1 \cap 2}+1}+\cdots+y_{s_{1}}\right)-\left(y_{s_{2}+1}+\cdots+y_{s_{1 \cup 2}}\right)+\frac{1}{2} \cdot s(b-2) \cdot\left(s_{1 \cup 2}+s_{2}-s_{1}-s_{1 \cap 2}\right) .
\end{aligned}
$$

à partir de quoi, on trouve en utilisant le lemme précédent :

$$
\begin{aligned}
f_{T_{1}}(y) & +f_{T_{2}}(y)-f_{T_{1} \cap T_{2}}(y)-f_{T_{1} \cup T_{2}}(y) \\
& \geqslant s \cdot\left[(b-1)\left(s_{2}-s_{1}\right)+b s-d+\frac{b}{2}\left(s_{1 \cup 2}-s_{1 \cap 2}-s_{2}+s_{1}\right)\right] \\
& \geqslant s \cdot\left((b-1)\left(s_{2}-s_{1}\right)+b s-d\right) .
\end{aligned}
$$

Si $s>0$, il est clair que la dernière expression est elle-aussi strictement positive. L'inégalité du lemme est donc vraie, et même stricte, dans ce cas. Si au contraire $s=0$, le majorant $s \cdot\left((b-1)\left(s_{2}-s_{1}\right)+b s-d\right)$ s'annule et l'inégalité du lemme en résulte encore. Dans ce dernier cas, l'égalité peut se produire, mais dire que $s=0$ revient à dire que $T_{1} \cap T_{2}=T_{1}$, i.e. $T_{1} \subset T_{2}$. Comme il est clair que, réciproquement, si $T_{1} \subset T_{2}$, l'inégalité du lemme est une égalité, on a bien démontré ce qui avait été annoncé.

On est maintenant prêt à démontrer la proposition 3.1. On suppose donc $b \geqslant b_{0}$. Si l'on excepte le cas $b=d=2$ que l'on vérifie à part à la main, on a $b>d$ et donc le lemme précédent s'applique. On considère des parties $T_{1}, \ldots, T_{d-1}$ de $\{1, \ldots, d\}$ vérifiant les hypothèses i) et ii) énoncées en amont des lemmes. On pose également $T_{d}=\{1, \ldots, d\}$ et on appelle $y$ l'unique point d'intersection des hyperplans affines d'équation $f_{T_{i}}(y)=0$ pour $i$ variant entre 1 et $d$. Quitte à renuméroter les $T_{i}$, on peut supposer que leurs cardinaux sont rangés par ordre croissant. Comme $y \in A_{Q_{\max }, g, \ell}$ par hypothèse, les nombres $f_{T_{i}} \cup T_{j}(y)$ et $f_{T_{i} \cap T_{j}}(y)$ sont positifs ou nuls pour tous indices $i$ et $j$. Ainsi, on est nécessairement dans le cas d'égalité du lemme précédent, ce qui signifie, dans le cas où $i<j$, que $T_{i} \subset T_{j}$ d'après l'hypothèse supplémentaire que nous avons faite sur les cardinaux. Ainsi, on obtient

$$
T_{1} \subset T_{2} \subset \cdots \subset T_{d-1} \subset T_{d}=\{1, \ldots, d\} .
$$

Par ailleurs, toutes les inclusions sont strictes car les hyperplans d'équation $f_{T_{i}}(y)=0$ ont un unique point d'intersection et que deux d'entre eux ne peuvent être confondus. On en déduit qu'il existe une permutation $w^{\star} \in \mathfrak{S}_{d}$ telle que $T_{i}=\left\{w^{\star}(1), w^{\star}(2), \ldots, w^{\star}(i)\right\}$ pour tout $i$. Il reste à calculer les coordonnées $\left(y_{1}, \ldots, y_{d}\right)$ du point $y$. Celles-ci sont solutions du système d'équations suivant :

$$
\left\{\begin{array}{l}
y_{1}-b y_{d+1-w^{\star}(1)}=d-1-b\left(w^{\star}(1)-1\right) \\
\left(y_{1}+y_{2}\right)-b\left(y_{d+1-w^{\star}(1)}+y_{d+1-w^{\star}(2)}\right)=d^{2}-4-b\left(w^{\star}(1)+w^{\star}(2)-3\right) \\
\vdots \\
\left(y_{1}+\cdots+y_{d-1}\right)-b\left(y_{d+1-w^{\star}(1)}+\cdots+y_{d+1-w^{\star}(d-1)}\right) \\
=d^{2}-(d-1)^{2}-b\left(w^{\star}(1)+\cdots+w^{\star}(d-1)-\frac{d(d-1)}{2}\right) \\
y_{1}+\cdots+y_{d}=0
\end{array}\right.
$$

En retranchant chaque équation de sa précédente, le système fournit les équations

$$
y_{i}-b y_{d+1-w^{\star}(i)}=d+1-2 i-b\left(w^{\star}(i)-i\right), \quad \text { pour } 1 \leqslant i \leqslant d .
$$

En notant $w$ la permutation inverse de $i \mapsto d+1-w^{\star}(i)$, et en posant $z_{i}=y_{i}-d-1+2 i$ pour tout $i$, celles-ci se réécrivent $z_{i}=w(i)-i+\frac{z_{w(i)}}{b}$ et donnent donc :

$$
z_{i}=w(i)-i+\frac{z_{w(i)}}{b}=w(i)-i+\frac{w^{2}(i)-w(i)}{b}+\frac{z_{w^{2}(i)}}{b^{2}}=\cdots=\sum_{n=0}^{\infty} \frac{w^{n+1}(i)-w^{n}(i)}{b^{n}} .
$$


Un calcul facile montre alors que $y=\vec{\rho}_{w}$. Réciproquement, pour montrer que $\vec{\rho}_{w}$ est un point extrémal, si

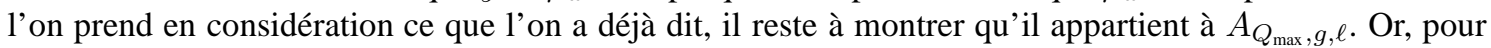
$T \subsetneq\{1, \ldots, d\}$, un nouveau calcul conduit à l'égalité suivante :

$$
\frac{f_{T}\left(\vec{\rho}_{w}\right)}{b-1}=\sum_{n=0}^{\infty} \frac{1}{b^{n}}\left(\sum_{t \in T} w^{n+1}(d+1-t)-\sum_{i=1}^{s} w^{n}(i)\right)
$$

où, comme d'habitude, $s$ est le cardinal de $T$. Chaque facteur $\sum_{t \in T} w^{n+1}(d+1-t)-\sum_{i=1}^{s} w^{n}(i)$ est, en valeur absolue, inférieur ou égal $s(d-1-s)$, qui est lui-même inférieur ou égal à $\left[\frac{(d-1)^{2}}{4}\right]=$ $b_{0}-1$. Ainsi, dans la somme infinie du membre de droite de l'égalité (3.4), la contribution des termes pour $n \geqslant 1$ est majorée en valeur absolue par $\frac{b_{0}-1}{b-1} \leqslant 1$. Par ailleurs, la contribution pour $n=0$ vaut $\sum_{t \in T} w(d+1-t)-\sum_{i=1}^{s} i$ qui est un nombre entier positif ou nul. S'il est strictement positif, il vaut au moins 1 et d'après ce qu'on a démontré précédemment, il ne peut être compensé par le reste de la somme; ainsi on a bien $f_{T}\left(\vec{\rho}_{w}\right) \geqslant 0$ dans ce cas. Si, maintenant, $\sum_{t \in T} w(d+1-t)=\sum_{i=1}^{s} i$, l'ensemble des $w(d+1-t)$ pour $t$ parcourant $T$ est nécessairement égal à l'ensemble $\{1, \ldots, s\}$. Il en résulte que pour tout $n$, on a aussi $\sum_{t \in T} w^{n+1}(d+1-t)=\sum_{i=1}^{s} w^{n}(i)$, et donc que $f_{T}\left(\vec{\rho}_{w}\right)$ est nul dans ce cas. Ainsi, pour tout sous-ensemble $T \subsetneq\{1, \ldots, d\}$, on a $f_{T}(y) \geqslant 0$. Comme on a également $y_{1}+\cdots+y_{d}=0$, on trouve bien $\vec{\rho}_{w} \in A_{Q_{\max }, g, \ell}$, et la proposition 3.1 est démontrée.

Lemme 3.4. On conserve les hypothèses de la proposition 3.1 et les hypothèses de sa démonstration. Alors, si $T$ n'est pas l'un des ensembles $T_{i}$, on a $f_{T}\left(\vec{\rho}_{w}\right)>0$.

En d'autres termes, le lemme dit qu'il ne concourt au sommet $\vec{\rho}_{w}$ que $d-1$ faces correspondant aux hyperplans d'équation $f_{T_{i}}(y)=0$ pour $1 \leqslant y \leqslant d-1$. (Notez bien que l'équation $f_{T_{d}}(y)=0$ définit l'hyperplan « somme des coordonnées égale $0 \gg$ dans lequel tout est plongé.)

Démonstration. Il suffit de remarquer que dans la somme de la formule (3.4), la contribution du terme correspondant à $n=d$ ! est positive ou nulle puisque $w^{d !}$ est l'identité. On en déduit que la contribution cumulée de tous les termes obtenus avec $n \geqslant 1$ est en fait strictement inférieure à $\frac{b_{0}-1}{b-1}$. Ainsi dès que l'ensemble des $w(d+1-t)(t \in T)$ n'est pas égal à $\{1, \ldots, s\}$, c'est-à-dire dès que $T$ n'est pas égal à $T_{s}$ avec $s=$ Card $T$, la quantité $f_{T}\left(\rho_{w}\right)$ est strictement positive.

\section{Un mot sur les points extrémaux de $A_{Q_{\max }, g, \ell}+C^{\star}$}

On démontrera dans la suite que $A_{Q, g, \ell}=A_{Q_{\max }, g, \ell}+C^{\star}$ (proposition 3.6) et, donc, plutôt que les points extrémaux de $A_{Q_{\max }, g, \ell}$, ce sont ceux de la somme $A_{Q_{\max }, g, \ell}+C^{\star}$ que l'on aimerait décrire. Ceux-ci forment un sous-ensemble des points extrémaux de $A_{Q_{\max }, g, \ell}$ qui, malheureusement, semble difficile à comprendre. On peut, malgré tout, définir un ordre sur $\mathfrak{S}_{d}$ qui permet de mieux appréhender la situation.

Voici comment on procède. Tout d'abord, pour tout entier $s \in\{1, \ldots, d\}$, on définit le préordre $\preccurlyeq s$ par :

$$
w_{1} \preccurlyeq s w_{2} \quad \operatorname{ssi}\left(\sum_{i=1}^{s} w_{1}^{n}(i)\right)_{n \geqslant 1} \leqslant \operatorname{lex}\left(\sum_{i=1}^{s} w_{2}^{n}(i)\right)_{n \geqslant 1}
$$

où $\leqslant_{\text {lex }}$ désigne l'ordre lexicographique sur l'ensemble des suites, qui donne le plus de poids aux petits indices. On pose ensuite :

$$
w_{1} \preccurlyeq w_{2} \quad \text { ssi } \quad w_{1} \preccurlyeq s w_{2} \text { pour tout } s \in\{1, \ldots, d\} \text {. }
$$

On vérifie facilement que $\preccurlyeq$ est bien un ordre sur $\mathfrak{S}_{d}$. À titre d'exemple, la figure 2 montre son diagramme de Hasse pour $d=4$ (sur cette figure, la permutation $w$ est notée $(w(1) w(2) w(3) w(4))$ ). Il existe déjà un certain nombre d'ordres sur le groupe des permutations $\mathfrak{S}_{d}$ (ordre de Bruhat, ordre faible, etc.) mais l'ordre $\preccurlyeq$ ne semble coïncider avec aucun d'entre eux (en tout cas, pas avec ceux qui sont connus de l'auteur). Pour l'instant, il est encore assez mystérieux. La proposition suivante explique malgré tout comment il est relié avec la problématique de cet article.

Proposition 3.5. On suppose $b \geqslant b_{0}$. Pour toutes permutations $w_{1}, w_{2} \in \mathfrak{S}_{d}$, on a $w_{1} \preccurlyeq w_{2}$ si, et seulement si $\vec{\rho}_{w_{1}} \in \vec{\rho}_{w_{2}}+C^{\star}$. 


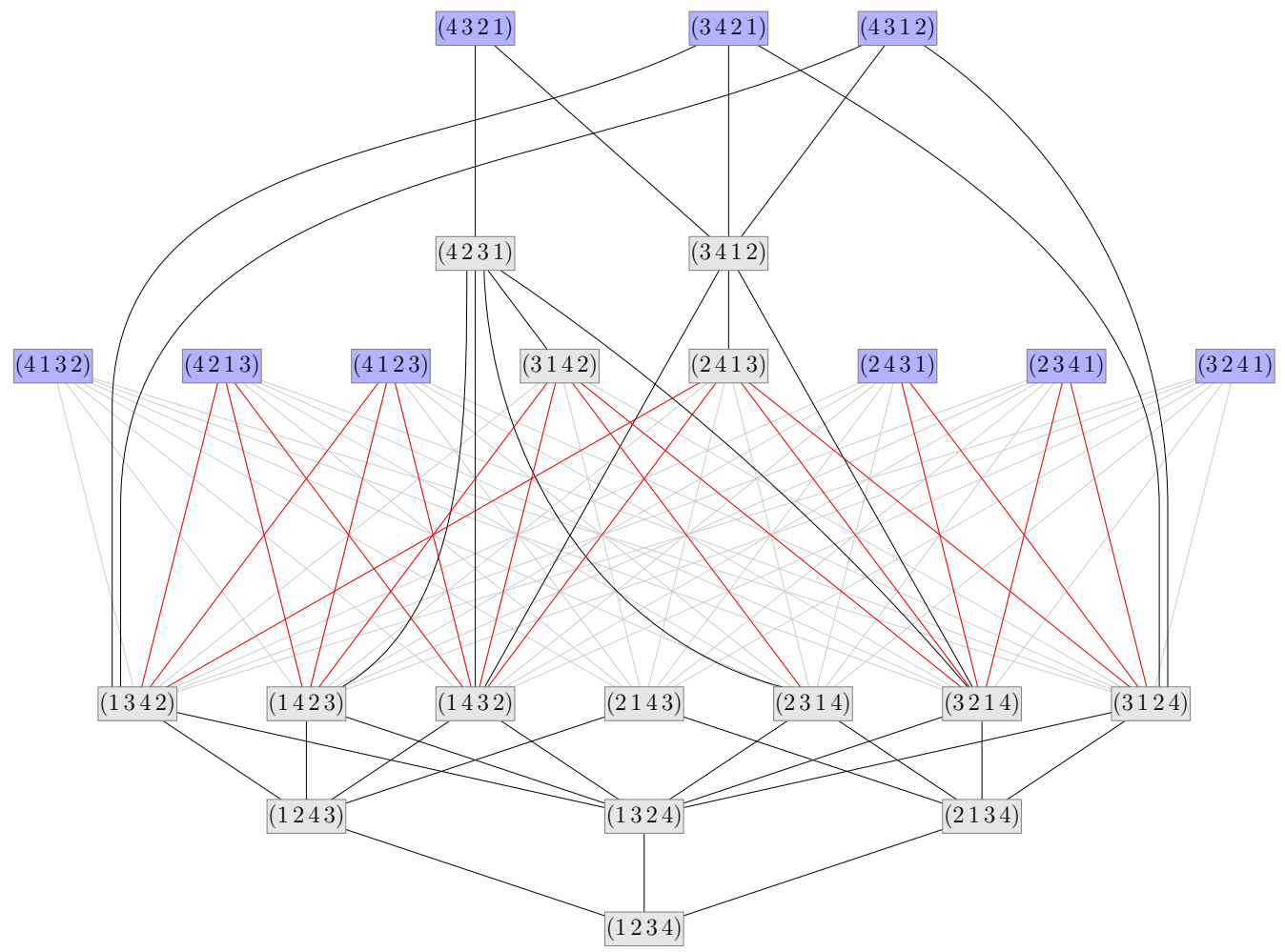

FIGURE 2 - Diagramme de Hasse de l'ordre $\preccurlyeq$ sur $\mathfrak{S}_{4}$

Les traits gris clairs sur la figure représentent, de la même façon que les traits noirs, des arêtes dans le graphe de Hasse. Attention : afin de faciliter la lecture, nous avons dessiné en rouge les arêtes entre une permutation du troisième étage et une du quatrième qui ne sont pas dans le diagramme de Hasse !

Les permutations coloriées en bleu sont les éléments maximaux.

Démonstration. Les coordonnées de $\vec{\rho}_{w_{1}}-\vec{\rho}_{w_{2}}$ sont

$$
(b-1) \cdot \sum_{n=1}^{\infty} \frac{w_{1}^{n}(i)-w_{2}^{n}(i)}{b^{n}}
$$

avec $1 \leqslant i \leqslant d$. Dire que ce vecteur est dans $C^{\star}$ revient à dire que, pour tout $s \in\{1, \ldots, d\}$, on a :

$$
\sum_{n=1}^{\infty} \frac{1}{b^{n}} \sum_{i=1}^{s}\left(w_{1}^{n}(i)-w_{2}^{n}(i)\right) \geqslant 0
$$

et que l'égalité a lieu si $s=d$. En fait, il est clair que l'égalité est toujours vérifiée pour $s=d$; on peut donc oublier cette condition. À part cela, on remarque que la somme $\sum_{i=1}^{s} w_{1}^{n}(i)-w_{2}^{n}(i)$ est majorée en valeur absolue par $b_{0}-1$. Comme $\frac{b_{0}-1}{b-1} \leqslant 1$, le signe de la somme infinie ne dépend que du signe du premier terme non nul. La proposition découle de cela.

Il résulte de la proposition que si deux permutations distinctes $w_{1}$ et $w_{2}$ sont telles que $w_{1} \preccurlyeq w_{2}$, alors $\vec{\rho}_{w_{1}}$ ne peut être un point extrémal de $A_{Q_{\max }, g, \ell}+C^{\star}$ (toujours sous l'hypothèse $b \geqslant b_{0}$ ). Ainsi, les seuls points extrémaux envisageables correspondent aux permutations $w$ qui sont des éléments maximaux pour $\preccurlyeq$. Par contre, il se peut que certains éléments maximaux ne définissent pas des points extrémaux de $A_{Q_{\max }, g, \ell}+C^{\star}$; lorsque $d=4$ par exemple, c'est le cas des vecteurs correspondant aux permutations (2 431 ) et (4213) (qui sont bien des éléments maximaux comme on le voit sur la figure ₹). 


\subsection{Le calcul de $A_{Q, g, \ell}$}

Le but de cette partie est de démontrer la proposition suivante.

Proposition 3.6. On suppose $b \geqslant b_{0}$. Alors $A_{Q, g, \ell}=A_{Q_{\max }, g, \ell}+C^{\star}$.

L'inclusion $A_{Q_{\max }, g, \ell}+C^{\star} \subset A_{Q, g, \ell}$ est facile. En effet, il est clair déjà que $A_{Q_{\max }, g, \ell}$ est inclus dans $A_{Q, g, \ell}$. Soient maintenant $y=\left(y_{1}, \ldots, y_{d}\right) \in A_{Q, g, \ell}$ et $z=\left(z_{1}, \ldots, z_{d}\right) \in C^{\star}$. Il suffit de montrer que $y+z \in A_{Q, g, \ell}$. Par définition de $A_{Q, g, \ell}$, on a $y_{1} \vec{\mu}_{1}+\cdots+y_{d} \vec{\mu}_{d}-\vec{\delta} \in Q^{\star}$, ce qui revient à dire que pour tout $q \in Q$,

$$
y_{1}\left\langle\vec{\mu}_{1} \mid q\right\rangle_{E}+\cdots+y_{d}\left\langle\vec{\mu}_{d} \mid q\right\rangle_{E} \geqslant\langle\vec{\delta} \mid q\rangle_{E} \cdot
$$

Par ailleurs, on sait que la suite des $\left\langle\vec{\mu}_{j} \mid q\right\rangle_{E}$ est décroissante en $j$; autrement dit elle définit un vecteur de $\mathbb{R}^{d}$ qui appartient à $C$. On en déduit que

$$
z_{1}\left\langle\vec{\mu}_{1} \mid q\right\rangle_{E}+\cdots+z_{d}\left\langle\vec{\mu}_{d} \mid q\right\rangle_{E} \geqslant 0 .
$$

Finalement en additionnant les formules (3.5) et (3.6), on trouve bien $y+z \in A_{Q, g, \ell}$ comme annoncé.

On se concentre désormais sur la démonstration de l'inclusion réciproque. À partir de maintenant, on suppose donc $b \geqslant b_{0}$. La démarche générale est la suivante. Tout d'abord, pour tout point extrémal $\vec{\rho}_{w}$ de $A_{Q_{\max }, g, \ell}$, on note $D_{w}$ le cône convexe tel qu'au voisinage de $\vec{\rho}_{w}$, on ait $A_{Q_{\max }, g, \ell}=\vec{\rho}_{w}+D_{w}$. Si $\vec{\rho}_{w}$ est un point extrémal de $A_{Q_{\max }, g, \ell}+C^{\star}$, les ensembles convexes $A_{Q_{\max }, g, \ell}+C^{\star}$ et $\vec{\rho}_{w}+D_{w}+C^{\star}$ coïncident encore au voisinage de $\vec{\rho}_{w}$. On en déduit que

$$
A_{Q_{\max }, g, \ell}+C^{\star}=\bigcap_{w \in \mathcal{S}_{d}}\left(\vec{\rho}_{w}+D_{w}+C^{\star}\right)
$$

où l'on a noté $\mathcal{S}_{d}$ le sous-ensemble de $\mathfrak{S}_{d}$ correspondant aux points extrémaux de $A_{Q_{\max }, g, \ell}+C^{\star}$. Ainsi, il suffit de montrer que tout élément de $A_{Q, g, \ell}$ appartient à $\vec{\rho}_{w}+D_{w}+C^{\star}$ pour tout $w \in \mathcal{S}_{d}$. On va en fait montrer que c'est le cas pour tout $w \in \mathfrak{S}_{d}$. Pour ce faire, l'idée est d'interpréter la somme $\vec{\rho}_{w}+D_{w}+C^{\star}$ comme des $A_{Q_{w}, g, \ell}$ pour certaines cônes convexes $Q_{w} \subset E$. Il suffira alors pour conclure de montrer que tous les $Q_{w}$ sont inclus dans $Q$.

\subsubsection{Les ensembles $Q_{w}$}

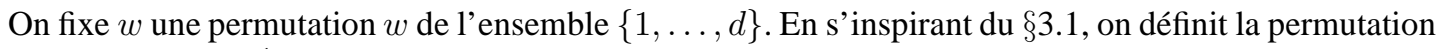
$w^{\star}: i \mapsto d+1-w^{-1}(i)$ et, pour tout $s$ compris entre 1 et $d$, on considère l'ensemble

$$
T_{s}(w)=\left\{w^{\star}(1), w^{\star}(2), \ldots, w^{\star}(s)\right\} .
$$

Cet ensemble correspond à une partie admissible de $I$ notée $I_{s}(w)$. On rappelle que, $s$ étant fixé, si on note $t_{1}, \ldots, t_{s}$ les nombres $w^{\star}(1), \ldots, w^{\star}(s)$ triés par ordre décroissant $\left(i . e . t_{1}>t_{2}>\cdots>t_{s}\right.$ ), alors le couple $(i, j)$ est dans $I_{s}(w)$ si, et seulement si $i \leqslant s$ et $j>d-t_{i}$. Lorsque $w$ est la permutation $\omega: i \mapsto d+1-i$, on a $w^{\star}=$ id et donc $T_{s}(\omega)=\{1, \ldots, s\}$; l'ensemble admissible $T_{s}(\omega)$ est alors simplement l'ensemble $I_{s}=\{(i, j) \in I \mid j-i \geqslant d-s\}$ qui a déjà été considérée. De façon générale, on vérifie facilement que les ensembles $I_{s}(w)$ définissent une partition croissante de $I$ telle que $I_{d}(w)=I$. On considère la fonction $\operatorname{ord}_{w}: I \rightarrow\{1, \ldots, d\}$ qui à un couple $(i, j)$ associe le plus petit entier $s$ tel que $(i, j) \in I_{s}(w)$. De façon évidente, pour tout $s$, l'ensemble $I_{s}(w)$ regroupe les éléments $x \in I$ tels que $\operatorname{ord}_{w}(x) \leqslant s$.

Pour se représenter les constructions précédentes, il est commode de considérer les éléments $(i, j)$ comme les cases d'un tableau triangulaire (voir figure 3). La fonction ord ${ }_{w}$ correspond alors à un remplissage des cases du tableau par les nombres entiers compris entre 1 et $d$. Celui-ci s'obtient en fait très simplement à partir de la permutation $w$, comme suit. On place d'abord sur la ligne du haut et dans les $w(1)$ dernières colonnes du tableau le nombre 1 . Ensuite, on place le nombre 2 dans les $w(2)$ dernières colonnes du tableau à chaque fois dans la case la plus haute qui n'est pas déjà remplie. Ainsi, si $w(2)<w(1)$, tous les nombres 2 se retrouvent sur la deuxième ligne, tandis que si $w(2)>w(1)$, on écrit $w(1)$ nombres 2 sous les 1 déjà écrits à l'étape précédente, et on met les $w(2)-w(1)$ nombres 2 restants sur la première ligne. On continue ensuite avec les 3 : on les place dans les $w(3)$ dernières colonnes, toujours le plus haut possible. Et ainsi de suite jusqu'à $d$. La figure 3 montre le remplissage obtenue pour la permutation (32 451$)$ (pour $d=5$ donc). 


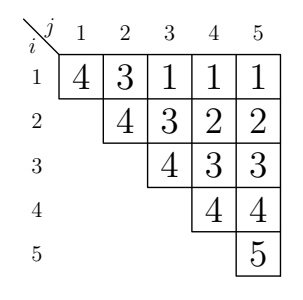

FIGURE 3 - La fonction ord $(32451)$

On définit encore un graphe $I_{w}$ comme suit : ses sommets sont les éléments de $I$ et l'on convient qu'il y a

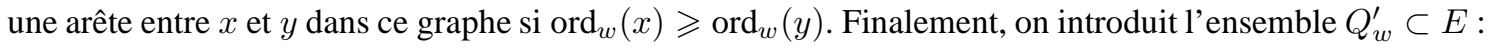
c'est le cône convexe $Q_{I_{w}}$ associé au graphe $I_{w}$ par la recette donnée juste en dessous de la définition 2.8. Pour $w_{0}: i \mapsto d+1-i$, l'ensemble $Q_{w_{0}}$ n'est autre que l'ensemble $Q_{\min }$.

Lemme 3.7. On suppose $b \geqslant b_{0}$. Alors, on a $A_{Q_{w}^{\prime}, g, \ell}=\vec{\rho}_{w}+D_{w}$.

Démonstration. Les parties admissibles du graphe $I_{w}$ (dans le sens de la définition 2.8) sont exactement les $I_{s}(w)$ pour $1 \leqslant s \leqslant d$. La proposition 2.9 assure donc que le cône dual $\left(Q_{w}^{\prime}\right)^{\star}$ est défini dans $E$ par les équations :

$$
\sum_{(i, j) \in I} x_{i, j}=0 \quad \text { et } \forall s \in\{1, \ldots, s\}, \quad \sum_{(i, j) \in I_{s}(w)} x_{i, j} \leqslant 0 .
$$

où les $x_{i, j}$ sont les coordonnées canoniques sur $E=\mathbb{R}^{I}$. En injectant cela dans la formule (2.1) et en utilisant le lemme 2.10, on trouve qu'un élément $y=\left(y_{1}, \ldots, y_{d}\right) \in \mathbb{R}^{d}$ appartient à $A_{Q_{w}^{\prime}, g, \ell}$ si, et seulement si $y_{1}+\ldots+y_{d}=0$ et $f_{I_{s}(w)}(y) \geqslant 0$ pour tout $s$ (où la fonction $f_{I_{s}(w)}$ est défini comme précédemment, voir formule (3.2)).

Par ailleurs, étant donné que $b \geqslant b_{0}$, le lemme 3.4 s'applique et implique que le cône $D_{w}$ est défini par les équations et inéquations :

$$
y_{1}+\cdots+y_{d}=0 \quad \text { et } \quad g_{I_{s}(w)}(y)=0
$$

où, si $T$ est une partie de $\{1, \ldots, d\}$, on a noté $g_{T}$ la fonction linéaire associé à $f_{T}$ : pour tout $y=$ $\left(y_{1}, \ldots, y_{n}\right)$ dans $\mathbb{R}^{d}$, on a $g_{T}(y)=y_{1}+\cdots+y_{s}-b \cdot \sum_{t \in T} y_{d+1-t}$ avec $s=$ Card $T$. Comme $\vec{\rho}_{w}$ a été justement construit pour vérifier $f_{I_{s}(w)}\left(\vec{\rho}_{w}\right)=0$ pour tout $s$, l'égalité du lemme en découle.

Soit $D$ le cône convexe de $E$ défini par $D=\left\{\left(q_{i, j}\right) \in E \mid \mu_{1,1} \geqslant \mu_{1,2} \geqslant \cdots \geqslant \mu_{1, d}\right\}$ où les $\mu_{i, j}$ sont définis à partir des $q_{i, j}$ par la formule (1.7). On rappelle que l'on a défini dans le $\S 2.3 .2$ des vecteurs $\vec{\mu}_{j} \in \mathbb{R}^{d}$ tels que $\mu_{1, j}=\left\langle\vec{\mu}_{j} \mid q\right\rangle_{E}$ où $q=\left(q_{i, j}\right) \in E$. Ainsi $D^{\star}$, le cône dual de $D$, n'est autre que le cône convexe engendré par les vecteurs $\vec{\mu}_{j}-\vec{\mu}_{j+1}$ pour $j$ parcourant l'ensemble d'indices $\{1, \ldots, d-1\}$. Par ailleurs, l'application $g$ est définie par $q \mapsto\left(\left\langle\vec{\mu}_{1} \mid q\right\rangle_{E}, \ldots,\left\langle\vec{\mu}_{1} \mid q\right\rangle_{E}\right)$ et donc envoie $D^{\star}$ sur l'ensemble des $y=\left(y_{1}, \ldots, y_{d}\right) \in \mathbb{R}^{d}$ tels que $y_{1} \geqslant \cdots \geqslant y_{d}$, c'est-à-dire $C$. On pose enfin $Q_{w}=Q_{w}^{\prime} \cap D \subset E$. Le cône dual de $Q_{w}$ est alors égal à $\left(Q_{w}^{\prime}\right)^{\star}+D^{\star}$. À partir de là et de ce qui a été dit précédemment (et notamment du lemme 3.7), il suit, en déroulant les définitions, que l'ensemble $A_{Q_{w}, g, \ell}$ est égal à $\vec{\rho}_{w}+D_{w}+C^{\star}$ comme souhaité. Il ne reste donc plus qu'à démontrer que $Q_{w} \subset Q$.

\subsubsection{Intermède : la permutation des perdants}

On constate sur l'exemple de la figure 3 que si l'on retire la ligne du haut du damier triangulaire, et que l'on soustrait 1 à tous les nombres restants, on obtient une numérotation qui correspond à une nouvelle permutation $w^{\prime}$, qui est ici égale à (2341). Dans ce paragraphe, on montre que cela est vrai de façon générale et on explique comment obtenir la permutation $w^{\prime}$ à partir de $w$.

Définition 3.8. Soit $w$ une permutation de l'ensemble $\{1, \ldots, d\}$.

Un record de $w$ est un entier $w(i)$ tel que $w(j)<w(i)$ pour tout $j<i$. Si $w(i)$ est un record de $w$, on dit qu'il apparaît en position $i$. 
La permutation des perdants de $w$ est la permutation $w^{\prime}$ de $\{1, \ldots, d-1\}$ définie par récurrence en décrétant que $w^{\prime}(i)$ est le plus petit élément de l'ensemble différence

$$
\{w(1), w(2), \ldots, w(i+1)\} \backslash\left\{w^{\prime}(1), \ldots, w^{\prime}(i-1)\right\} .
$$

La notion de record est classique : elle a déjà été introduite il y a de nombreuses années dans [14] et a depuis fait l'objet de multiples études, notamment en ce qui concerne leur distribution asymptotique. Par contre, l'auteur n'a pas réussi à trouver une trace antérieure de la permutation des perdants.

On montre immédiatement par récurrence que l'ensemble (3.7) est toujours de cardinal 2 (i.e. que l'ensemble que l'on ôte est toujouts inclus dans le premier), et plus précisément qu'il contient exactement l'élément $w(i+1)$, et le dernier record de $w$ apparaissant avant $i$. Ainsi si $w(i+1)$ n'est pas un record de $w$, on a $w^{\prime}(i)=w(i+1)$, tandis que dans le cas contraire, $w^{\prime}(i)$ est le record précédent de $w$. Dans le cas de la permutation $w=(32451)$, on voit que les records de $w$ sont les entiers 3 , 4 et 5 et que la permutation est perdants de $w$ est $w^{\prime}=(2341)$.

On peut donner une reformulation moins mathématique des définitions précédentes qui donne tout son sens à la terminologie. Il faut pour cela imaginer que les entiers de 1 à $d$ sont des candidats qui prennent part à une compétition, dans l'ordre indiqué par la permutation $w$ : si l'on reprend notre exemple, cela signifie que 3 joue d'abord, 2 juste après, etc. En outre un candidat est d'autant plus fort au jeu que l'entier qui lui est attaché est grand. Les records correspondent alors aux records au sens usuel : l'entier 3 joue en premier, et donc décroche le record (il n'a pas grand mérite, mais peu importe); ensuite vient le tour de l'entier 2 dont la performance est moins bonne, il n'a donc pas le record; ensuite, joue 4 qui fait un nouveau record; puis 5 qui bat encore le record; et enfin 1 qui n'améliore certainement pas le record. Les records successifs sont donc bien 3,4 et 5 .

La permutation des perdants, quand à elle, s'interprète comme suit. Il faut imaginer qu'au fur et à mesure que la compétition se déroule, on met à jour une liste des perdants. Au premier tour, l'entier 3 joue et il n'y a pour l'instant aucun perdant; on écrit donc rien sur la liste. Ensuite, c'est au tour de l'entier 2 de jouer; celui-ci fait un moins bon résultat et se retrouve ainsi être le premier perdant. C'est maintenant 4 qui s'élance, et il subtilise le record à 3 ; l'entier 3 devient comme ceci un perdant et on l'inscrit sur la liste en dessous de 2. Et ainsi de suite, on obtient la liste des perdants — ou la permutation des perdants pour reprendre la terminologie mathématique - composée dans l'ordre des nombres $2,3,4$ et 1 .

Dans la suite, et notamment lors des démonstrations, on continuera d'employer la terminologie imagée issue de la métaphore de la compétition.

Lemme 3.9. Soient $w \in \mathfrak{S}_{d}$ et $w^{\prime}$ sa permutation des perdants. Soit $\operatorname{ord}_{w^{\prime}}: I^{\prime} \rightarrow\{1, \ldots, d-1\}$ (avec $\left.I^{\prime}=\left\{(i, j) \in \mathbb{N}^{2} \mid 1 \leqslant i \leqslant j \leqslant d-1\right\}\right)$ la fonction associée à $w^{\prime}$. Alors $\operatorname{ord}_{w^{\prime}}(i, j)=\operatorname{ord}_{w}(i+1, j+1)-1$.

Démonstration. On raisonne sur la représentation de la fonction $\operatorname{ord}_{w}$ sous forme de tableau triangulaire (voir figure 3). Il s'agit alors de montrer qu'un entier $i \geqslant 2$ apparaît exactement $w(i)-w^{\prime}(i-1)$ fois sur la première ligne. Si $w(i)$ n'est pas un record, alors manifestement $i$ n'apparaît pas sur la première ligne et on a déjà dit qu'alors $w(i)=w^{\prime}(i-1)$. Dans ce cas, on a donc bien ce que l'on voulait. Si, au contraire, $w(i)$ est un record, soit $w(j)$ le record de $w$ qui apparaît juste avant i, i.e. $w(j)=\max \{w(1), \ldots, w(i-1)\}$. Alors $w^{\prime}(i-1)=w(j)$ et l'entier $i$ apparaît $w(i)-w(j)$ sur la première ligne du tableau. On a donc, à nouveau, bien vérifié ce que l'on avait annoncé.

\subsubsection{La fin de la démonstration}

On en revient à la démonstration de la proposition 3.6. Il reste à démontrer que $Q_{w} \subset Q$ pour toute permutation $w \in \mathfrak{S}_{d}$. On fixe à partir de maintenant $w \in \mathfrak{S}_{d}$ et un élément $q=\left(q_{i, j}\right) \in Q_{w}$. On note également $\mu_{i, j}$ les nombres réels définis par la formule (1.7). Par hypothèse $\mu_{1, j} \geqslant \mu_{2, j} \geqslant \cdots \geqslant \mu_{d, j}$ et, en examinant la définition de $Q_{w}^{\prime}$, on prouve qu'il existe des réels $q_{1} \leqslant q_{2} \leqslant \cdots \leqslant q_{d}$ tels que $q_{i, j}=q_{\text {ord }_{w}(i, j)}$. On veut montrer que $q \in Q$, ce qui signifie que les inégalités $\mu_{i, j} \leqslant \mu_{i+1, j}$ et $\mu_{i, j} \geqslant \mu_{i+1, j+1}$ sont satisfaites pour dès que cela a un sens. La première étape consiste à exprimer les $\mu_{i, j}$ en fonction des $q_{i}$. Pour cela, on considère $w_{1}, \ldots, w_{d}$ les permutations définies par récurrence en convenant que $w_{1}=w$ et que $w_{i+1}$ est la permutation des perdants de $w_{i}$; ainsi $w_{i}$ est une permutation de l'ensemble $\{1, \ldots, d+1-i\}$. On définit encore les permutations $w_{i}^{\vee} \in \mathfrak{S}_{d+1-i} \operatorname{par} w_{i}^{\vee}(j)=w_{i}^{-1}(d+2-i-j)$ où $w_{i}^{-1}$ désigne bien sûr la permutation inverse de $w_{i}$. 
Lemme 3.10. Avec les notations précédentes, on a pour tout couple $(i, j) \in I$ :

$$
\mu_{i, j}=b q_{w_{i}^{\vee}(j-i+1)+i-1}-q_{j} .
$$

Démonstration. D'après le lemme 3.9 et les expressions donnant les valeurs de $\mu_{i, j}$, il suffit de traiter le cas où $i=1$. La formule à démontrer s'écrit alors simplement $\mu_{1, j}=b q_{w_{1}^{\vee}(j)}-q_{j}$. Par définition, on a :

$$
\begin{aligned}
\mu_{1, j} & =-q_{j, d}+b \cdot \sum_{i=1}^{j} q_{i, j}-b \cdot \sum_{i=0}^{j-1} q_{i, j-1} \\
& =-q_{\operatorname{ord}_{w}(j, d)}+b \cdot \sum_{i=1}^{j} q_{\operatorname{ord}_{w}(i, j)}-b \cdot \sum_{i=0}^{j-1} q_{\operatorname{ord}_{w}(i, j-1)} .
\end{aligned}
$$

Or, si l'on se souvient de l'interprétation de la fonction $\operatorname{ord}_{w}$ en termes de remplissage de tableau, il est clair que $\operatorname{ord}_{w}(j, d)=j$ et que l'ensemble $\left\{\operatorname{ord}_{w}(1, j), \ldots, \operatorname{ord}_{w}(j, j)\right\}$ consiste en les entiers $s$ tels que $w(s) \geqslant d+1-j$. Ainsi, trouve-t-on :

$$
\left\{\operatorname{ord}_{w}(1, j), \ldots, \operatorname{ord}_{w}(j, j)\right\}=\left\{\operatorname{ord}_{w}(1, j-1), \ldots, \operatorname{ord}_{w}(j-1, j-1)\right\} \cup\left\{w_{1}^{\vee}(j)\right\}
$$

et la formule du lemme en découle.

On est en mesure à présent de montrer que $\mu_{1, j} \leqslant \mu_{2, j}$ pour tout indice $j \in\{2, \ldots, d\}$. Par définition de $w_{2}^{\vee}$, on a $w_{2} \circ w_{2}^{\vee}(j-1)=d+1-j$; autrement dit, l'entier $d+1-j$ apparait en position $w_{2}^{\vee}(j-1)$ dans la permutation des perdants de $w$. Il a donc forcément joué avant le tour $w_{2}^{\vee}(j-1)+1$, ce qui se traduit mathématiquement par l'inégalité $w_{1}^{\vee}(j) \leqslant w_{2}^{\vee}(j-1)+1$. Ainsi, grâce au lemme 3.10, on obtient :

$$
\mu_{1, j}=b q_{w_{1}^{\vee}(j)}-q_{j} \leqslant b q_{w_{2}^{\vee}(j-1)+1}-q_{j}=\mu_{2, j}
$$

ce qui est bien ce que l'on désirait. La démonstration de l'inégalité $\mu_{1, j} \geqslant \mu_{2, j+1}$ suit une idée analogue. On remarque d'abord que, d'après le lemme 3.10 , la différence entre les deux nombres à comparer s'exprime comme suit :

$$
\mu_{1, j}-\mu_{2, j+1}=b\left(q_{w_{1}^{\vee}(j)}-q_{w_{2}^{\vee}(j)+1}\right)+q_{j+1}-q_{j} .
$$

Comme $q_{j+1} \geqslant q_{j}$, l'égalité que l'on souhaite démontrer est trivialement satisfaite si $q_{w_{1}^{\vee}(j)} \geqslant q_{w_{2}^{\vee}(j)+1}$ et donc dès que $w_{1}^{\vee}(j)>w_{2}^{\vee}(j)$. On suppose donc à partir de maintenant que $w_{1}^{\vee}(j) \leqslant w_{2}^{\vee}(j)$. On pose $i_{1}=w_{1}^{\vee}(j)$ et $i_{2}=w_{2}^{\vee}(j)$; on a alors $w_{1}\left(i_{1}\right)=d+1-j$ et $w_{2}\left(i_{2}\right)=d-j$. Selon la permutation $w$, l'entier $d-j$ a donc forcément joué avant le temps $i_{2}+1$. Par ailleurs, il n'a pas pu jouer avant le temps $i_{1}$ car sinon, il aurait été battu par $d+1-j$ au temps $i_{1}$ et donc aurait au pire rejoint la liste des perdants en position $i_{1}-1$. De même, il n'a pas pu jouer en $i_{1}$ car c'était alors le tour de $d+1-j$, ni entre les temps $i_{1}+1$ et $i_{2}$ car il aurait été alors perdant tout de suite (étant donné que $d+1-i$ aurait déjà fait une meilleure performance avant). On en déduit que $d-j$ a joué au temps $i_{2}+1$, c'est-à-dire que $w\left(i_{2}+1\right)=d-j$, soit encore $w_{1}^{\vee}(j+1)=i_{2}+1=w_{2}^{\vee}(j)+1$. En appliquant le lemme 3.10, on obtient

$$
\mu_{1, j+1}=b q_{w_{1}^{\vee}(j+1)}-q_{j+1}=b q_{w_{2}^{\vee}(j)+1}-q_{j+1}=\mu_{2, j+1}
$$

et la conclusion résulte alors de l'hypothèse $\mu_{1, j} \geqslant \mu_{1, j+1}$.

À présent, des deux égalités que l'on vient de prouver, il suit $\mu_{2, j} \geqslant \mu_{1, j} \geqslant \mu_{2, j+1}$ pour tout $j \in$ $\{2, \ldots, d-1\}$. On peut donc reitérer l'argumentation précédente en décalant les indices (ou, si l'on préfère, appliquer ce que l'on vient de démontrer à la permutation $w^{\prime}$ ) afin d'obtenir les inégalités $\mu_{2, j} \leqslant \mu_{3, j}$ pour $3 \leqslant j \leqslant d$, et $\mu_{2, j} \geqslant \mu_{3, j+1}$ pour $2 \leqslant j \leqslant d-1$. En continuant ainsi, on démontre bien au final ce que l'on voulait.

\subsection{Démonstration des théorèmes 2,4 et 5}

\subsubsection{Le cas des variétés $\mathcal{X}_{\mu}$}

On commence par démontrer le théorème 4 . Soit $\mu=\left(\mu_{1}, \ldots, \mu_{d}\right)$ un $d$-uplet d'entiers vérifiant $\mu_{1} \geqslant$ $\cdots \geqslant \mu_{d}$. En passant au déterminant, on démontre tout de suite que si $b-1$ ne divise pas $\mu_{1}+\cdots+\mu_{d}$, la variété $\mathcal{X}_{\mu}$ est vide. On suppose désormais que $\mu_{1}+\cdots+\mu_{d}$ est un multiple de $b-1$. On a

$$
\operatorname{dim}_{k} \mathcal{X}_{\mu}=\max \operatorname{dim}_{k} \mathcal{X}_{\varphi}
$$


où le maximum est pris sur tous les $\varphi \in \Phi_{\mathbb{Z}}$ tels que $\mu_{1, i}(\varphi)=\mu_{i}$ pour tout $i$. Les congruences énoncées sont donc une conséquence immédiate du corollaire 1.17 et du théorème 1.18. Pour le reste, on rappelle que l'on se restreint au cas où $h \geqslant 0$. On a donc $\operatorname{dim}_{k} \mathcal{X}_{\mu}=b_{Q, R, g, \ell, 0}^{\prime}(\mu)$, et, en vertu de la proposition 2.3, on est amené à justifier les deux assertions suivantes :

- l'image de $R \cap Q_{\mathbb{R}}$ (où on rappelle que $Q_{\mathbb{R}}$ est défini comme le $\mathbb{R}$-espace vectoriel engendré par $Q$ ) par la fonction $g$ contient l'ensemble des $d$-uplets $\left(y_{1}, \ldots, y_{d}\right)$ tels que $b-1$ divise $y_{1}+\cdots+y_{d}$;

- le nombre $b_{Q, g, \ell, 0}(\mu)=a_{Q, g, \ell}(\mu)$ est égal au minimum qui apparaît dans l'énoncé du théorème $\sharp$. Il est facile de construire un élement $q=\left(q_{i, j}\right)$ telle que toutes les inégalités des jeux I, II et III définissant $Q$ soient strictes; on pourra pour cela s'inspirer de l'exemple de la figure 1. Un tel point est dans l'intérieur de $Q$, ce qui assure que $Q$ est d'intérieur non vide et donc que $Q_{\mathbb{R}}=E$. Si l'on note $\vec{v}_{1}, \ldots, \vec{v}_{d}$ la base canonique de $\mathbb{R}^{d}$, un calcul facile montre qu'étant donné un entier $s$ compris entre 1 et $d$, l'image par $g$ de l'élément $\left(q_{i, j}\right) \in R$ défini par $q_{i, j}=\delta_{(i, j),(s, d)}$ ( $\delta$ étant le symbole de Kronecker) est $\vec{w}_{s}=b \vec{v}_{d}-\vec{v}_{s}$. En outre, un vecteur $y$ de coordonnées $\left(y_{1}, \ldots, y_{d}\right)$ se décompose sur la familles de $\vec{w}_{s}$ comme suit :

$$
y=\frac{b}{b-1} \cdot\left(y_{1}+\cdots+y_{d}\right) \cdot \vec{w}_{d}-\left(y_{1} \vec{w}_{1}+\cdots+y_{d} \vec{w}_{d}\right)
$$

Ainsi on trouve que $y$ appartient à $g(R)=g\left(R \cap Q_{\mathbb{R}}\right)$ dès que $b-1$ divise la somme de ses coordonnées $y_{1}+\cdots+y_{d}$, ce qui démontre la première assertion.

On en vient à la seconde. Comme l'on ne considère que des éléments $\mu$ dans $C$, on a $a_{Q, g, \ell}(\mu)=$ $b_{Q, g, \ell, 0, C}(\mu)$ et de même $a_{Q_{\max }, g, \ell}(\mu)=b_{Q_{\max }, g, \ell, 0, C}(\mu)$. D'après la proposition 2.2 et la description des points extrémaux de $A_{Q_{\max }, g, \ell}$ donnée par la proposition 3.1, il suffit de montrer que $B_{Q, g, \ell, 0, C}=$ $B_{Q_{\max }, g, \ell, 0, C}$, i.e. $A_{Q, g, \ell}+C^{\star}=A_{Q_{\max }, g, \ell}+C^{\star}$, ce qui suit de la proposition 3.6.

Remarque 3.11. Bien entendu, $a_{Q, g, \ell}(\mu)$ s'écrit aussi comme le minimum des produits scalaires $\langle\alpha \mid \mu\rangle_{d}$ où $\alpha$ décrit l'ensemble des points extrémaux de $A_{Q, g, \ell}$. Cela signifie que le théorème 4 reste vrai si l'on se contente de prendre le minimum sur le sous-ensemble (strict) $\mathcal{S}_{d}$ de $\mathfrak{S}_{d}$, qui est défini au début du $\S_{3.2}$. En d'autres termes, les permutations qui n'appartiennent pas à $\mathcal{S}_{d}$ ne contribuent jamais (i.e. pour aucun $\mu \in C)$ au minimum.

Il reste encore à démontrer que lorsque $\mu$ est $b$-régulier, le minimum des produits scalaires $\left\langle\vec{\rho}_{w} \mid \mu\right\rangle_{d}$ (pour $w$ décrivant $\mathfrak{S}_{d}$ ) est atteint lorsque $w=w_{0}: i \mapsto d+1-i$, et qu'il vaut alors $\frac{1}{b+1} \cdot\langle 2 \vec{\rho} \mid \mu\rangle_{d}$ (on rappelle que $\vec{\rho}$ est défini par $\left.\vec{\rho}=\left(\frac{d-1}{2}, \frac{d-3}{2}, \ldots, \frac{1-d}{2}\right) \in \mathbb{R}^{d}\right)$. On note pour cela Reg le sous-ensemble de $\mathbb{R}^{d}$ formé des éléments $\mu=\left(\mu_{1}, \ldots, \mu_{d}\right)$ qui sont $b$-réguliers; il s'agit d'un cône convexe dont le cône dual est noté Reg ${ }^{\star}$. Avec la proposition 2.2, on voit aisément qu'il suffit de démontrer le lemme suivant.

Lemme 3.12. Soit $w_{0}$ la permutation $i \mapsto d+1-i$. On a l'égalité :

$$
A_{Q_{w_{0}}, g, \ell}=A_{Q_{\min }, g, \ell}=\frac{2 \vec{\rho}}{b+1}+\operatorname{Reg}^{\star}
$$

Démonstration. On a déjà dit que les convexes $A_{Q_{w_{0}}, g, \ell}$ et $A_{Q_{\min }, g, \ell}$ étaient égaux. Pour $i \in\{1, \ldots, d-1\}$, on note $\vec{v}_{i} \in \mathbb{R}^{d}$ le vecteur $\vec{v}_{i}=(0, \ldots, 0,1,-1,0 \ldots, 0)$ où le 1 est en $i$-ème position. Ces vecteurs forment une base de l'hyperplan «somme des coordonnées égale $0 \gg$, noté $H$. D'après la définition des points $b$-réguliers, le cône dual Reg* est le cône convexe engendré par les vecteurs $\vec{w}_{i}=b \vec{v}_{d-i}-\vec{v}_{i}$ pour $1 \leqslant i \leqslant d-1$. Par ailleurs, comme dans la démonstration du lemme 3.7, on obtient qu'un élément $y=\left(y_{1}, \ldots, y_{d}\right) \in \mathbb{R}^{d}$ appartient à $A_{Q_{w_{0}}, g, \ell}$ si, et seulement si

$$
\left\{\begin{array}{l}
y_{1}+y_{2}+\cdots+y_{d}=0 \\
\left(y_{1}+\cdots+y_{s}\right)-b\left(y_{d+1-s}+\cdots+y_{d}\right) \geqslant s(d-s), \quad \forall s \in\{1, \ldots, d-1\} .
\end{array}\right.
$$

Comme le vecteur $\frac{2 \vec{\rho}}{b+1}$ n'est autre que l'intersection de ces $d$ hyperplans (vérification immédiate), le convexe $-\frac{2 \vec{\rho}}{b+1}+A_{Q_{w_{0}}, g, \ell}$ est le cône défini dans $H$ par les inégalités $\left(y_{1}+\cdots+y_{s}\right)-b\left(y_{d+1-s}+\cdots+y_{d}\right) \geqslant 0$ pour $s \in\{1, \ldots, d-1\}$. Pour conclure, il suffit de montrer que ce cône est engendré par les vecteurs $\vec{w}_{i}$. Or, il est engendré par les intersections $(d-2)$ à $(d-2)$ des $(d-1)$ hyperplans $($ de $H)$ frontière des demi-espaces précédents. Un calcul direct montre enfin que ces intersections sont exactement les vecteurs $\vec{w}_{i}$, ce qui conclut. 


\subsubsection{Le cas des variétés $\mathcal{X}_{\leqslant \mu}$}

On en vient maintenant aux variétés $\mathcal{X}_{\leqslant \mu}$, c'est-à-dire à la démonstration du théorème兯. Pour ce faire, on suit à nouveau la même méthode : on donne une description de l'ensemble convexe $B_{Q, g, \ell, C^{\star}}$, à partir

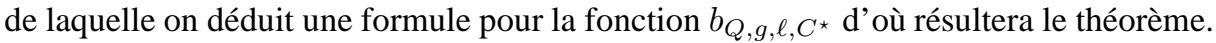

Le calcul de $A_{Q, g, \ell} \cap C$ Le lemme 3.12 donne une description explicite de l'ensemble $A_{Q_{\min }, g, \ell}$. Dans ce paragraphe, on se propose de démontrer qu'il s'en déduit une autre description simple de l'intersection $A_{Q, g, \ell} \cap C$, au moins si $b$ est suffisamment grand. Plus précisément, on se propose de démontrer que, si $b \geqslant b_{0}$, on a :

$$
A_{Q, g, \ell} \cap C=A_{Q_{\min }, g, \ell} \cap C=\left(\frac{2 \vec{\rho}}{b+1}+\operatorname{Reg}^{\star}\right) \cap C
$$

D'après la proposition 3.6 (en fait, seule l'inclusion facile sert), il suffit d'établir l'égalité $\left(A_{Q_{\max }, g, \ell}+C^{\star}\right) \cap$ $C=A_{Q_{\min }, g, \ell} \cap C$. On commence par un lemme concernant les points extrémaux de $A_{Q_{\max }, g, \ell}$.

Lemme 3.13. On suppose $b \geqslant \max \left(b_{0}, d+1\right)$. Alors le seul point extrémal de $A_{Q_{\max }, g, \ell}$ qui appartient à $C$ est $\frac{2 \vec{\rho}}{b+1}$.

Démonstration. Comme on a supposé $b \geqslant b_{0}$, la proposition 3.1 s'applique et les points extrémaux de $A_{Q_{\max }, g, \ell}$ sont de la forme $\vec{\rho}_{w}$. On est ainsi ramené à montrer que le vecteur $\vec{\rho}_{w}$ n'appartient pas à $C$ dès que $w$ n'est pas la permutation $i \mapsto d+1-i$. Or, par définition de $C$ et d'après la formule donnant les coordonnées de $\vec{\rho}_{w}$, le fait que $\vec{\rho}_{w}$ soit élément de $C$ implique que pour tout $i \in\{1, \ldots, d-1\}$, l'égalité suivante est satisfaite :

$$
\sum_{n=1}^{\infty} \frac{w^{n}(i+1)-w^{n}(i)+1}{b^{n}} \geqslant 0 .
$$

Du fait que $b \geqslant d+1$, ceci implique que le premier terme de la somme (i.e. pour $n=1$ ) est lui-même positif ou nul. Ainsi $w(i+1) \geqslant w(i)-1$ pour tout indice $i \in\{1, \ldots, d-1\}$ et il est alors clair que $w$ ne peut être que la permutation $i \mapsto d+1-i$.

Lemme 3.14. Soit $y=\left(y_{1}, \ldots, y_{d}\right)$ un élément de $A_{Q_{\min }, g, \ell}$ tel que $y_{i} \leqslant y_{i+1}+1$ pour tout $i \in\{1, \ldots, d-$ $1\}$. Alors $y \in A_{Q_{\max }, g, \ell}$.

Démonstration. D'après la descrition de $A_{Q_{\max }, g, \ell}$ donnée par le système (3.1), il s'agit de montrer que $f_{T}(y) \geqslant 0$ pour toute partie $T$ de $\{1, \ldots, d\}$. Soit $T$ une telle partie. Si on note $t_{1}<\cdots<t_{s}$ ses éléments (avec $s=$ Card $T$ ), l'inégalité $f_{T}(y) \geqslant 0$ devient

$$
y_{1}+\cdots+y_{s}-b \cdot \sum_{i=1}^{s} y_{d+1-t_{i}} \geqslant s(d-s)+b \cdot \sum_{i=1}^{s}\left(t_{i}-i\right) .
$$

Par ailleurs, comme $y$ est pris dans $A_{Q_{\min }, g, \ell}$, on a

$$
y_{1}+\cdots+y_{s}-b \cdot \sum_{i=1}^{s} y_{d+1-t_{i}} \geqslant s(d-s)+b \cdot \sum_{i=1}^{s}\left(y_{d+1-i}-y_{d+1-t_{i}}\right) .
$$

Or, on a $t_{i} \geqslant i$ pour tout $i$ d'où, d'après l'hypothèse, on tire $y_{d+1-i}-y_{d+1-t_{i}} \leqslant t_{i}-i$. L'inégalité (3.9) s'ensuit.

On peut à présent démontrer l'égalité (3.8) lorsque $b \geqslant \max \left(b_{0}, d+1\right)$. On rappelle qu'il suffit de montrer que le polytope $P_{1}=A_{Q_{\min }, g, \ell} \cap C$ est inclus dans le polytope $P_{2}=\left(A_{Q_{\max }, g, \ell}+C^{\star}\right) \cap C$ et, pour cela, on peut se contenter de prouver que tous les sommets de $P_{1}$ (en incluant ceux à l'infini) sont dans $P_{2}$. On vérifie tout de suite que Reg $\subset C^{\star}$; ainsi $C \subset \operatorname{Reg}^{\star}$ et la description donnée par le lemme 3.12 implique $A_{Q_{\max }, g, \ell}+C=A_{Q_{\max }, g, \ell}$ et finalement $P_{1}+C=P_{1}$. Comme en plus $P_{1} \subset C$, on en déduit que les sommets à l'infini de $P_{1}$ sont ceux de $C$. Mais du fait que $C \subset C^{\star}$ (vérification facile), il suit que $P_{2}+C=P_{2}$. Ainsi les sommets à l'infini de $C$ sont bien dans $P_{2}$.

Il reste donc à traiter le cas des sommets de $P_{1}$ à distance fini, c'est-à-dire des points extrémaux de $P_{1}$. Soient $F_{1}, \ldots, F_{d-1}$ les facettes de $A_{Q_{\min }, g, \ell}$; ce sont des cônes simpliciaux de dimension $d-2$ issus de $\frac{2 \vec{p}}{b+1}$. Le lemme 3.14 assure que les convexes $A_{Q_{\max }, g, \ell}+C^{\star}$ et $A_{Q_{\min }, g, \ell}$ sont égaux sur un voisinage de $\frac{2 \vec{p}}{b+1}$. 
On en déduit que $A_{Q_{\max }, g, \ell}+C^{\star}$ a exactement $d-1$ facettes issues de $\frac{2 \vec{\rho}}{b+1}$ et que l'on peut numéroter cellesci $F_{1}^{\prime}, \ldots, F_{d-1}^{\prime}$ de sorte que $F_{i}^{\prime} \subset F_{i}$ pour tout $i$. Pour chaque $i$, les sommets de $F_{i}^{\prime}$ à distance finie sont

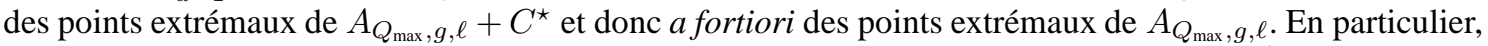
d'après la proposition 3.1 et le lemme 3.13 , ceux-ci ne sont pas dans $C$ à l'exception de $\frac{2 \vec{\rho}}{b+1}$. Les sommets à l'infini, quant à eux, correspondent aux directions de Reg ${ }^{\star}$, c'est-à-dire aux vecteurs $\vec{w}_{i}$ introduits dans la démonstration du lemme 3.12. Comme aucun des $\vec{w}_{i}$, ni de leurs opposés, n'est dans $C$, on en déduit finalement que $F_{i} \cap C=F_{i}^{\prime} \cap C$.

Il est maintenant facile de conclure : tout point extrémal de $P_{1}=A_{Q_{\min }, g, \ell} \cap C$ appartient à l'une des faces $F_{i}$ et bien sûr également à $C$. Il appartient donc à la face $F_{i}^{\prime}$ correspondante, et par suite à $A_{Q_{\max }, g, \ell}+C^{\star}$ puis à $P_{2}$.

Obtention de la majoration On sait que la dimension de la variété $\mathcal{X}_{\leqslant \mu}$ est majorée par la quantité $b_{Q, g, \ell, C^{\star}}(\mu)$ qui d'après la proposition 2.2 vaut $\inf _{\alpha \in B_{Q, g, \ell, C^{\star}}}\langle\alpha \mid \mu\rangle_{E}$ où, on rappelle que $B_{Q, g, \ell, C^{\star}}=$ $A_{Q, g, \ell} \cap C$. Pour démontrer la première majoration :

$$
\operatorname{dim}_{k} \mathcal{X}_{\leqslant \mu} \leqslant \frac{\langle 2 \vec{\rho} \mid \mu\rangle_{d}}{b+1}
$$

il suffit donc de prouver que le vecteur $\frac{2 \vec{\rho}}{b+1}$ est dans $B_{Q, g, \ell, C^{\star}}$. Il est déjà évident qu'il appartient à $C$. De plus, le lemme 3.12 montre qu'il appartient à $A_{Q_{\min }, g, \ell}$, d'où il suit, grâce au lemme 3.14, qu'il est aussi dans $A_{Q_{\max }, g, \ell}$ et donc a fortiori dans $A_{Q, g, \ell}$. On en vient finalement à la deuxième majoration du théorème : on suppose $b \geqslant \max \left(b_{0}, d+1\right)$ et on veut montrer

$$
\operatorname{dim}_{k} \mathcal{X}_{\leqslant \mu} \leqslant \sup _{\substack{\mu^{\prime} \leqslant \mu \\ \mu^{\prime} \in \operatorname{Reg}}} \frac{\left\langle 2 \vec{\rho} \mid \mu^{\prime}\right\rangle_{d}}{b+1}
$$

Si l'on définit la forme linéaire $L: \mathbb{R}^{d} \rightarrow \mathbb{R}, \mu \mapsto \frac{1}{b+1} \cdot\langle 2 \vec{\rho} \mid \mu\rangle_{d}$, on remarque que le majorant dans (3.10) n'est autre que $b_{\mathrm{Reg}, \mathrm{id}, L, C^{\star}}(\mu)$. Pour terminer la preuve, il suffit donc de montrer que $b_{\mathrm{Reg}, \text { id }, L, C^{\star}}=$ $b_{Q, g, \ell, C^{\star}}$. Or, en déroulant les définitions, on trouve $A_{\mathrm{Reg}, \text { id }, L}=\frac{2 \vec{\rho}}{b+1}+\operatorname{Reg}^{\star}$ et donc $A_{\mathrm{Reg}, \text { id }, L}=A_{Q_{\min }, g, \ell}$ d'après le lemme 3.12. L'égalité (3.8) implique alors $A_{\mathrm{Reg}, \mathrm{id}, L} \cap C=A_{Q, g, \ell} \cap C$ à partir de quoi la proposition 2.2 permet de conclure.

Obtention de la minoration Il ne reste plus qu'à démontrer la minoration. Étant donné que si $\mu^{\prime} \leqslant \mu$, on a tautologiquement $\mathcal{X}_{\leqslant \mu^{\prime}} \subset \mathcal{X}_{\leqslant \mu}$ et $\operatorname{donc}_{\operatorname{dim}_{k}} \mathcal{X}_{\leqslant \mu^{\prime}} \leqslant \operatorname{dim}_{k} \mathcal{X}_{\leqslant \mu}$, il suffit de montrer que si $\mu=$ $\left(\mu_{1}, \ldots, \mu_{d}\right)$ est fortement intégralement $b$-régulier, alors :

$$
\operatorname{dim}_{k} \mathcal{X}_{\leqslant \mu} \geqslant \frac{\langle 2 \vec{\rho} \mid \mu\rangle_{d}}{b+1}-(d-1)^{2}-\frac{(d-2)^{2}}{4} .
$$

Pour cela, il suffit de trouver un élément $q \in Q \cap R$ tel que $g(q) \in \mu-C^{\star}$ et $\ell(q) \geqslant \frac{\langle 2 \vec{\rho} \mid \mu\rangle_{d}}{b+1}-(d-$ $1)^{2}-\frac{(d-2)^{2}}{4}$. On pose, pour tout $i, q_{i}^{\prime}=\frac{\mu_{i}+b \mu_{d+1-i}}{b^{2}-1}$. La condition de divisibilité qui apparaît dans la définition d'un $d$-uplet fortement intégralement $b$-régulier assure que si $q_{1}^{\prime}+\cdots+q_{d}^{\prime}$ est un entier alors que les conditions d'inégalité se traduisent par $q_{1}^{\prime} \leqslant q_{2}^{\prime} \leqslant \cdots \leqslant q_{d-1}^{\prime} \leqslant q_{d}^{\prime}-d$. Pour $i<d$, on note $q_{i}$ la partie entière supérieure de $q_{i}^{\prime}$ (i.e. le plus petit entier plus grand ou égal à $q_{i}^{\prime}$ ) et on définit $q_{d}$ de sorte que $q_{1}+\cdots+q_{d}=q_{1}^{\prime}+\cdots+q_{d}^{\prime}$. Tous les $q_{i}$ sont alors des entiers, et on vérifie directement que la suite qu'ils forment est croissante. Ainsi le vecteur $q$ de coordonnées $q_{i, j}=q_{d-j+i}$ (pour $(i, j) \in I$ ) appartient à $Q$. En outre, un calcul immédiat montre que pour tout $s$, on a :

$$
\begin{aligned}
\left\langle\vec{\mu}_{1}+\cdots+\vec{\mu}_{s} \mid q\right\rangle_{E} & =b\left(q_{d}+\cdots+q_{d+1-s}\right)-\left(q_{1}+\cdots+q_{s}\right) \\
& =-b\left(q_{1}+\cdots+q_{d-s}\right)-\left(q_{1}+\cdots+q_{s}\right) \\
& \leqslant-b\left(q_{1}^{\prime}+\cdots+q_{d-s}^{\prime}\right)-\left(q_{1}^{\prime}+\cdots+q_{s}^{\prime}\right) \\
& =b\left(q_{d}^{\prime}+\cdots+q_{d+1-s}^{\prime}\right)-\left(q_{1}^{\prime}+\cdots+q_{s}^{\prime}\right)=\mu_{1}+\cdots+\mu_{s}
\end{aligned}
$$

et l'égalité est atteinte lorsque $s=d$. Autrement dit, on a bien $g(q) \in \mu-C^{\star}$. Il reste à minorer $\ell(q)$ :

$$
\ell(q)=\langle\vec{\delta} \mid q\rangle_{d}=\sum_{i=1}^{d}(2 i-d-1) q_{i}=\frac{\langle 2 \vec{\rho} \mid \mu\rangle_{d}}{b+1}+\sum_{i=1}^{d}(2 i-d-1)\left(q_{i}-q_{i}^{\prime}\right)
$$


Or $q_{d}-q_{d}^{\prime}$ est compris entre $1-d$ et 0 , tandis que les $q_{i}-q_{i}^{\prime}$ pour $i<d$ sont compris entre 0 et 1 . On en déduit que :

$$
\ell(q) \geqslant \frac{\langle 2 \vec{\rho} \mid \mu\rangle_{d}}{b+1}-(d-1)^{2}-\sum_{i=2}^{d} \max (2 i-d-1,0) \geqslant \frac{\langle 2 \vec{\rho} \mid \mu\rangle_{d}}{b+1}-(d-1)^{2}-\frac{(d-2)^{2}}{4} .
$$

\subsubsection{Le cas des variétés $\mathcal{X}_{\leqslant e}$}

Preuve de la majoration Soit $e$ un nombre entier. La variété $\mathcal{X}_{\leqslant e}$ s'écrit manifestement comme l'union disjointe des variétés $\mathcal{X}_{\leqslant \mu}$ pour $\mu=\left(\mu_{1}, \cdots, \mu_{d}\right)$ vérifiant $e \geqslant \mu_{1} \geqslant \cdots \geqslant \mu_{d} \geqslant 0$. Il suffit donc de démontrer que, si $\mu$ est tel qu' on vient de le décrire, la dimension de $\mathcal{X}_{\leqslant \mu}$ est majorée par $\left[\frac{d^{2}}{4}\right] \cdot \frac{e}{b+1}$. Or, par le théorème 5, on sait que cette dimension est majorée par $\frac{1}{b+1} \cdot\langle 2 \vec{\rho} \mid \mu\rangle_{d}$ et on a

$$
\frac{\langle 2 \vec{\rho} \mid \mu\rangle_{d}}{b+1}=\sum_{i=1}^{d} \frac{d+1-i}{b+1} \cdot \mu_{i} \leqslant e \cdot \sum_{i=1}^{[d / 2]} \frac{d+1-i}{b+1}=\left[\frac{d^{2}}{4}\right] \cdot \frac{e}{b+1} .
$$

La conclusion en résulte.

Preuve de la minoration Pour obtenir la minoration, on doit estimer la quantité $b_{Q, R, f, \ell, C}^{\prime}(e, 0)$ f. Or, il est évident par définition que si $q \in Q \cap R$ vérifie $f(q) \in(e, 0)+C$, cette quantité est minorée par $\ell(q)$. Il suffit donc, pour terminer la démonstration du théorème 2 , de construire un élément $q=\left(q_{i, j}\right) \in Q \cap R$ tel que :

$$
f(q) \in(e, 0)+C \quad \text { et } \quad \ell(q)=\left[\frac{d^{2}}{4}\right] \cdot\left[\frac{e-b+2}{b+1}\right] .
$$

Soient $n=\left[\frac{e-b+2}{b+1}\right]$ et $m$ le reste de la division euclidienne de $(-n)$ par $b-1$. On considère l'élément $q$ défini comme suit :

$$
\begin{array}{ll}
q_{i, j}=\frac{m+b n}{b-1} & \text { si } 0 \leqslant j-i<\frac{d}{2} \\
q_{i, j}=\frac{m+n}{b-1} \quad \text { si } j-i \geqslant \frac{d}{2} .
\end{array}
$$

Il est clair que $\frac{m+b n}{b+1} \geqslant \frac{m+n}{b+1}$. À partir de là et du fait que la valeur de $q_{i, j}$ ne dépend que de la différence $j-i$, il résulte que $q$ appartient à $Q_{\min }$, et donc a fortiori à $Q$. Comme, par ailleurs, il suit de la définition de $m$ que tous les $q_{i, j}$ sont entiers, on a bien $q \in Q \cap R$. D'autre part, un calcul direct donne $\left\langle\vec{\mu}_{1} \mid q\right\rangle_{E}=$ $m+n(b+1) \leqslant b-2+e-b+2=e$ et $\left\langle\vec{\mu}_{d} \mid q\right\rangle_{E}=m \geqslant 0$, d'où il suit $f(q) \in(e, 0)+C$. Finalement, à nouveau un calcul facile conduit à la valeur souhaitée pour $\ell(q)=\langle\vec{\delta} \mid q\rangle_{E}$.

\subsection{Points extrémaux de $A_{Q, g, \ell} \cap C$ : quelques exemples}

J'aimerais revenir un instant sur la démonstration de la majoration du théorème 5 . Elle procédait ainsi. Dans un premier temps, on a utilisé le théorème 1.18 afin de majorer la dimension de $\mathcal{X}_{\leqslant \mu}$ par la quantité $b_{Q, g, \ell, C^{\star}}(\mu)$, et ensuite on a prouvé l'égalité $b_{Q, g, \ell, C^{\star}}(\mu)=b_{\mathrm{Reg}, \mathrm{id}, L, C^{\star}}(\mu)$ en montrant que chacun de ces deux nombres s'égalisait avec:

$$
M(\mu)=\inf _{\alpha \in K}\langle\alpha \mid \mu\rangle_{d} \quad \text { où } \quad K=\left(\frac{2 \vec{\rho}}{b+1}+\operatorname{Reg}^{\star}\right) \cap C .
$$

Ainsi, l'expression $M(\mu)$ est une nouvelle façon d'exprimer le majorant obtenu. En outre, $K$ est un polytope qui n'a qu'un nombre fini de points extrémaux, et si on appelle $\Lambda$ leur ensemble, on a :

$$
M(\mu)=\inf _{\lambda \in \Lambda}\langle\lambda \mid \mu\rangle_{d}
$$

pour tout $\mu \in \operatorname{Reg}+C^{\star}=C^{\star}$, c'est-à-dire dès que $\mu_{1} \geqslant \cdots \geqslant \mu_{d}$ si, comme d'habitude, on appelle $\mu_{i}$ $(1 \leqslant i \leqslant d)$ les coordonnées de $\mu$.

6. Remarquez que la proposition 2.3 et la discussion qui suit sa démontration nous dit qu'elle diffère de $b_{Q, f, \ell, C}(e, 0)$ d'une quantité bornée. Cependant, pour arriver à la minoration énoncée dans le théorème日, on a besoin d'être plus précis que cela. 
Déterminer les points extrémaux de $K$ apparaît donc comme une question naturelle et importante. Malheureusement, bien que $K$ soit défini de façon plutôt simple (c'est l'intersection de $2 d-2$ demi-espaces affines dans un espace de dimension $d-1$ ), la combinatoire de ses points extrémaux paraît compliquée. Par exemple, le nombre de ces points semble exploser très rapidement lorsque $d$ augmente. Ces deux dernières impressions se sont forgées à la suite de calculs numériques effectués grâce au logiciel polymake [3]. La deuxième ligne du tableau 11 donne, par exemple, le nombre de points extrémaux de $K$ pour diverses valeurs de $d$ avec $b=10000$ (la valeur de $b$ importe peu pour la complexité de l'ensemble des points extrémaux de $K$ comme l'explique le théorème 3.15 ci-après). La croissance est apparemment exponentielle; on notera qu'elle ne peut, en tout cas, pas être pire car, un point extrémal étant situé à l'intersection de $d$ hyperplans parmi les $2 d$ définissant $K$, leur nombre est trivialement majoré par $\left(\begin{array}{c}2 d-2 \\ d-1\end{array}\right) \leqslant 4^{d-1}$.

\begin{tabular}{|l||c|c|c|c|c|c|c|c|c|}
\hline Dimension $d$ & 2 & 3 & 4 & 5 & 6 & 7 & 8 & 9 & 10 \\
\hline $\begin{array}{l}\text { Nombre de points } \\
\text { extrémaux de } K\end{array}$ & 1 & 3 & 6 & 15 & 33 & 70 & 136 & 347 & 667 \\
\hline $\begin{array}{l}\text { Nombre de points } \\
\text { extrémaux de } K+C^{\star}\end{array}$ & 1 & 3 & 5 & 9 & 17 & 31 & 47 & 103 & 163 \\
\hline
\end{tabular}

TABLE 1 - Nombre de points extrémaux de $K$ et $K+C^{\star}$ pour $b=10000$

Cependant, les points extrémaux que l'on a trouvé précédemment ne sont pas vraiment tous pertinents. En effet, comme l'on ne s'intéresse aux variétés $\mathcal{X}_{\leqslant \mu}$ que lorsque les coordonnées de $\mu$ sont triés par ordre décroissant c'est-à-dire lorsque $\mu \in C$, on peut, au lieu de considérer la fonction $b_{Q, f, \ell, C^{\star}}$, travailler plutôt avec la fonction $b_{Q, f, \ell, C^{\star}, C}$ définie par:

$$
\begin{aligned}
b_{Q, g, \ell, C^{\star}, C}(y) & =b_{Q, g, \ell, C^{*}}(y) & & \text { si } y \in C \\
& =-\infty & & \text { sinon. }
\end{aligned}
$$

Par la proposition 2.2, on a

$$
M^{\prime}(\mu)=\inf _{\alpha \in K^{\prime}}\langle\alpha \mid \mu\rangle_{d} \quad \text { où } \quad K^{\prime}=K+C^{\star}
$$

Ainsi, plutôt que décrire les points extrémaux de $K$, on a plutôt envie de comprendre ceux de $K+C^{\star}$ qui forment un sous-ensemble (en général strict) des points extrémaux de $K$. Si l'on reprend les exemples précédents ( $b=10000, d$ petit), on constate sur le tableau 1, que l'on élimine en effet ainsi un bon paquet de points extrémaux, au moins pour les petites valeurs de $d$.

Il a été dit précédemment que la dépendance en $b$ est moins délicate à comprendre. En effet, on a le théorème suivant.

Théorème 3.15. On pose, comme précédemment,

$$
K(b)=\left(\frac{2 \vec{\rho}}{b+1}+\operatorname{Reg}^{\star}\right) \cap C \quad \text { et } \quad K^{\prime}(b)=K(b)+C^{\star}
$$

et on note $\Lambda(b)$ et $\Lambda^{\prime}(b)$ l'ensemble des points extrémaux de $K(b)$ et $K^{\prime}(b)$ respectivement. Alors, pour $b$ suffisamment grand, le cardinal de $\Lambda(b)$ (resp. $\Lambda^{\prime}(b)$ ) est constant et les coordonnées de points de cet ensemble s'expriment comme des fractions rationnelles en $b$.

Démonstration. Le théorème résulte du fait que les méthodes de calcul de points extrémaux de polytopes s'appliquent dans n'importe quel corps ordonné. Ici donc, on peut voir $K(b)$ et $K^{\prime}(b)$ comme des polytopes définis sur le corps réel $\mathbb{R}(b)$ muni de l'ordre qui fait de $b$ un élément infiniment grand. Ces polytopes ont alors bien sûr un nombre de sommets qui ne dépend pas de $b$ et les coordonnées de ces sommets sont des éléments de $\mathbb{R}(b)$, c'est-à-dire des fractions rationnelles en $b$. Il reste à justifier que ces expressions redonnent bien les points extrémaux de $K(b)$ et $K^{\prime}(b)$ lorsque l'on spécialise $b$ en une valeur suffisamment grande. Mais c'est évident car le calcul des sommets faits dans $\mathbb{R}(b)$ est valable dès que $b$ satisfait un certain nombre fini d'inégalités, et donc en particulier dès que $b$ est suffisamment grand.

Le tableau 2 montre les fractions rationnelles que l'on obtient pour les petites valeurs de $d$.

\footnotetext{
7. En fait, si $\mu$ est quelconque, on montre qu'il existe $\mu^{\prime}=\left(\mu_{1}^{\prime} \geqslant \cdots \mu_{d}^{\prime}\right)$, facilement explicitable à partir de $\mu$, tel que $\mathcal{X}_{\leqslant \mu}=$ $\mathcal{X}_{\leqslant \mu^{\prime}}$
} 


\begin{tabular}{|c|c|c|}
\hline Dimension $d$ & $\begin{array}{c}\text { Domaine de } \\
\text { validité }\end{array}$ & Points extrémaux de $K+C^{\star}$ \\
\hline 2 & $b \geqslant 2$ & $\frac{2 \vec{\rho}}{b+1}=\frac{(1,-1)}{b+1}$ \\
\hline 3 & $b \geqslant 2$ & $\begin{array}{c}\frac{2 \vec{\rho}}{b+1}=\frac{(2,0,-2)}{b+1} \\
\frac{(-2,-2,4)}{b+2} \\
\frac{(4,-2,-2)}{b+2}\end{array}$ \\
\hline 4 & $b \geqslant 3$ & $\begin{array}{c}\frac{2 \vec{\rho}}{b+1}=\frac{(3,1,-1,-3)}{b+1} \\
\frac{(3,3,3,-9)}{b+3} \\
\frac{(9,-3,-3,-3)}{b+3} \\
\frac{(1,-1,-1,1)}{b-1}+\frac{(4,0,0,-4)}{b+1} \\
\frac{(-1,1,1,-1)}{b-1}+\frac{(4,0,0,-4)}{b+1}\end{array}$ \\
\hline 5 & $b \geqslant 6$ & $\begin{array}{c}\frac{2 \vec{\rho}}{b+1}=\frac{(4,2,0,-2,-4)}{b+1} \\
\frac{(4,4,4,4,-16)}{b+4} \\
\frac{(16,-4,-4,-4,-4)}{b+4} \\
\frac{(3,-2,-2,-2,3)}{b-1}+\frac{(7,0,0,0,-7)}{b+1} \\
\frac{(-3,2,2,2,-3)}{b-1}+\frac{(7,0,0,0,-7)}{b+1} \\
\frac{(-2,2,0,0,0)}{b}+\frac{(6,0,0,0,-6)}{b+1} \\
\frac{(0,0,0,-2,2)}{b}+\frac{(6,0,0,0,-6)}{b+1} \\
\frac{(4,0,0,0,-4)}{b+1}+\frac{(0,2,2,-4,0)}{b+2} \\
\frac{(4,0,0,0,-4)}{b+1}+\frac{(0,4,-2,-2,0)}{b+2}\end{array}$ \\
\hline
\end{tabular}

TABLE 2 - Coordonnées des points extrémaux de $K+C^{\star}$ 


\section{Perspectives et conjectures}

\subsection{Peut-on espérer une formule exacte pour la dimension?}

Si $h \geqslant 0$, le théorème 1.18 donne une formule exacte pour la dimension des variétés $\mathcal{X}_{\varphi}$. On peut donc raisonnablement penser que, dans ce cas, il est possible d'en déduire une formule exacte pour la dimension de $\mathcal{X}_{\leqslant e}, \mathcal{X}_{\mu}$ et $\mathcal{X}_{\leqslant \mu}$. Et de fait, on dispose d'une telle formule car on peut toujours écrire (comme nous l'avons déjà fait plusieurs fois) :

$$
\operatorname{dim}_{k} \mathcal{X}_{\leqslant e}=b_{Q, R, f, \ell, \mathbb{R}^{+} \times \mathbb{R}^{-}}^{\prime}(0, e)=\sup _{\substack{q \in Q \cap R \\ f(q) \in(e, 0)-\left(\mathbb{R}^{+} \times \mathbb{R}^{-}\right)}} \ell(q)
$$

ainsi que des expressions analogues pour les autres variétés. Les notations dans la formule (4.1) sont celles qui ont été utilisées dans les sections précédentes; on renvoie le lecteur aux débuts des $\$ \S 2.2$ et 2.3.2 pour un récapitulatif rapide des définitions. Lorsque $h \geqslant 0$, calculer la dimension de $\mathcal{X}_{\leqslant e}$ revient ainsi à calculer le nombre $b_{Q, R, f, \ell, \mathbb{R}^{+} \times \mathbb{R}^{-}}^{\prime}(0, e)$. Comme ce dernier s'exprime comme le maximum d'une forme linéaire sur un ensemble fini on peut, en un certain sens, considérer que le problème est résolu ; en tout cas, il est aisé à partir de là d'écrire un algorithme qui répond à la question pour des entiers $d, b$ et un $d$-uplet $\mu$ donnés. Toutefois, cela n'est pas entièrement satisfaisant car l'on aimerait comprendre par exemple le comportement précis de la dimension de $\mathcal{X}_{\leqslant e}$ lorsque les paramètres $d, b$ et $e$ varient. Pour ce type de questions, l'approche algorithmique naïve, que l'on vient de présenter, s'avère insuffisante. À l'opposé de cette approche algorithmique, il y a un théorème général de logique qui prédit la dépendance de $b_{Q, R, f, \ell, \mathbb{R}^{+} \times \mathbb{R}^{-}}^{\prime}(0, e)$ en fonction de $e$. Voici ce qu'il implique dans notre cas.

Théorème 4.1. On suppose que $h \neq 0$. Alors, il existe un entier $N$ et une fonction $f: \mathbb{Z} / N \mathbb{Z} \rightarrow \mathbb{Q}$ tel que, pour e suffisamment grand, on ait :

$$
\operatorname{dim}_{k} \mathcal{X}_{\leqslant e}=\left[\frac{d^{2}}{4}\right] \cdot \frac{e}{b+1}+f(e \bmod N)
$$

Démonstration. D'après la formule (4.1), la différence $\delta(e)=\operatorname{dim}_{k} \mathcal{X}_{\leqslant e}-\left[\frac{d^{2}}{4}\right] \cdot \frac{e}{b+1}$ est définie par une formule de l'arithmétique de Presburger ayant la variable libre $e$. Par le théorème d'élimination des quantificateurs dans l'arithmétique de Presburger, cette formule est équivalente à une formule sans quantificateurs. Par ailleurs, le théorème 2 montre que la fonction $\delta: e \mapsto \delta(e)$ est bornée sur $\mathbb{N}$. On déduit facilement à partir de là que, pour $e$ suffisamment grand, elle ne dépend que de la réduction de $e$ modulo un certain entier $N$. C'est exactement ce qu'il fallait démontrer.

On peut reformuler le théorème précédent en disant que la série génératrice

$$
\sum_{e=0}^{\infty}\left(\operatorname{dim}_{k} \mathcal{X}_{\leqslant e}\right) \cdot X^{e}
$$

est, en fait, une fraction rationnelle. Ce théorème est probablemement intéressant sur le plan théorique mais, d'une point de vue pratique, le théorème 4.1 est absolument inutile car il ne dit rien ni sur l'entier $N$, ni sur la fonction $f$, ni sur le moment à partir duquel la formule pour la dimension est correcte. On souligne en outre, au cas où l'énoncé n'était pas clair sur ce point, que ces données dépendent $a$ priori de $d$ et de $b$. Un élément positif malgré tout est qu'il existe des algorithmes pour les calculer. Par contre, malheureusement, au delà de la dimension 3 (pour laquelle on peut encore faire les calculs à la main), il n'est pas envisageable d'utiliser de tels outils, ceux-ci étant (à l'heure actuelle) trop peu efficaces.

Le théorème 4.1 admet, bien sûr, des analogues pour les variétés $\mathcal{X}_{\mu}$ et $\mathcal{X}_{\leqslant \mu}$ qui sont peu ou prou équivalents à la rationnalité des séries

$$
\sum_{\mu \in \mathbb{N}^{d}}\left(\operatorname{dim}_{k} \mathcal{X}_{\mu}\right) \cdot X_{1}^{\mu_{1}} X_{2}^{\mu_{2}} \cdots X_{d}^{\mu_{d}} \quad \text { et } \quad \sum_{\mu \in \mathbb{N}^{d}}\left(\operatorname{dim}_{k} \mathcal{X}_{\leqslant \mu}\right) \cdot X_{1}^{\mu_{1}} X_{2}^{\mu_{2}} \cdots X_{d}^{\mu_{d}}
$$

où les entiers $\mu_{i}$ désignent les coordonnées de $\mu$. 


\subsubsection{Calcul en petites dimensions}

En guise d'illustration du résultat du théorème 4.1 (ou plutôt de l'un de ses analogues qui viennent d'être évoqués), on se propose de calculer les dimensions exactes des variétés $\mathcal{X}_{\mu}$ lorsque $d=2$ et également lorsque $d=3$ dans certains cas. Pour cela, plutôt que d'utiliser les $q_{i, j}$ pour paramétrer les $d$-uplet $\varphi=$ $\left(\varphi_{1}, \ldots, \varphi_{d}\right)$ comme cela a été fait jusqu'à présent, on va plutôt travailler ici avec les $\mu_{i, j}$, ce qui sera plus commode. Les inégalités qui définissent l'ensemble $Q$ s'écrivent

$$
\begin{array}{r}
\mu_{i-1, j-1} \leqslant \mu_{i, j} \leqslant \mu_{i-1, j} \quad \text { et } \quad b \mu_{i, i}+b \sum_{s=i+1}^{j}\left(\mu_{i, s}-\mu_{i+1, s}\right)+\sum_{s=j+1}^{d}\left(\mu_{i, s}-\mu_{i+1, s}\right) \\
\leqslant b \mu_{i-1, i-1}+b \sum_{s=i}^{j-1}\left(\mu_{i-1, s}-\mu_{i, s}\right)+\sum_{s=j}^{d}\left(\mu_{i-1, s}-\mu_{i, s}\right)
\end{array}
$$

pour tout couple d'entiers $(i, j)$ avec $2 \leqslant i \leqslant j \leqslant d$, alors que les conditions d'intégrité, qui définissent le réseau $R$, sont données par la proposition 1.11 :

$$
\begin{aligned}
\forall(i, j) \in I, & \mu_{i, j} \in \mathbb{Z} \\
\forall i \in\{1, \ldots, d\}, & \mu_{i, i}+\mu_{i, i+1}+\cdots+\mu_{i, d} \equiv 0 \quad(\bmod b-1) .
\end{aligned}
$$

On rappelle que si $\varphi$ est l'élément de $\Phi$ correspondant à une donnée $\left(\mu_{i, j}\right)$ satisfaisant les conditions précédentes, alors pour tout réseau $L \subset M$, les exposants des diviseurs élémentaires du $k[[u]]$-module engendré par $\sigma(L)$ par rapport à $L$ sont les $\mu_{i}=\mu_{1, i}$ (voir propositions 1.2 et 1.7) et que :

$$
\operatorname{dim}(\varphi)=\sum_{j=1}^{d}(d+1-j) \cdot \mu_{1, j}-\sum_{(i, j) \in I} \mu_{i, j}=\sum_{j=1}^{d}(d+1-j) \cdot \mu_{j}-\sum_{(i, j) \in I} \mu_{i, j}
$$

(voir lemme 1.15). Dans la suite, on supposera toujours que $h \geqslant 0$ de sorte que la quantité précédente s'égalise avec la dimension de la variété $\mathcal{X}_{\varphi}$. On note $\lceil x\rceil$, la partie entière supérieure du nombre réel $x$, c'est-à-dire le plus petit entier supérieur ou égal à $x$. On pose aussi def $(x)=\lceil x\rceil-x$; c'est à l'évidence un nombre compris entre 0 et 1 qui ne dépend que de la congruence de $x$ modulo $\mathbb{Z}$.

En dimension 2 D'après ce que l'on vient de rappeler, étant donnés des nombres entiers $\mu_{1} \geqslant \mu_{2}$ tels que $b-1$ divise $\mu_{1}+\mu_{2}$, calculer la dimension de la variété $\mathcal{X}_{\left(\mu_{1}, \mu_{2}\right)}$ revient à maximiser le nombre $\mu_{1}-\mu_{1,2}$ sous les contraintes

$$
\left\{\begin{array}{l}
\mu_{1} \leqslant \mu_{1,2} \leqslant \mu_{2} \\
(b+1) \mu_{1,2} \geqslant b \mu_{1}+\mu_{2} \\
b-1 \text { divise } \mu_{1,2}
\end{array}\right.
$$

En écrivant $\mu_{1,2}=(b-1) x$, on voit tout de suite que le maximum cherché est atteint pour $x=\left\lceil\frac{b \mu_{1}+\mu_{2}}{b^{2}-1}\right\rceil$. Ainsi, obtient-on :

$$
\operatorname{dim}_{k} \mathcal{X}_{\left(\mu_{1}, \mu_{2}\right)}=\mu_{1}-(b-1) \cdot\left\lceil\frac{b \mu_{1}+\mu_{2}}{b^{2}-1}\right\rceil=\frac{\mu_{1}-\mu_{2}}{b+1}-(b-1) \cdot \operatorname{def}\left(\frac{b \mu_{1}+\mu_{2}}{b^{2}-1}\right)
$$

On constate immédiatement sur la dernière écriture que la dimension de $\mathcal{X}_{\left(\mu_{1}, \mu_{2}\right)}$ s'exprime comme la somme de $\frac{\mu_{1}-\mu_{2}}{b+1}$ (qui correspond au terme attendu) et d'un terme correctif qui ne dépend que des congruences de $\mu_{1}$ et $\mu_{2}$ modulo $b^{2}-1$. De surcroît, ce terme correctif varie dans l'intervalle $\left.] 1-b, 0\right]$; la dimension de $\mathcal{X}_{\left(\mu_{1}, \mu_{2}\right)}$ se caractérise donc encore comme le plus grand entier $\leqslant \frac{\mu_{1}-\mu_{2}}{b+1}$ qui est congru à $\mu_{1}$ modulo $b-1$ (en accord avec la congruence du théorème $\uparrow$ ).

La dimension de la variété $\mathcal{X}_{\leqslant e}$, quant à elle, s'obtient en prenant le maximum de $\operatorname{dim}_{k} \mathcal{X}_{\left(\mu_{1}, \mu_{2}\right)}$ sur tous les couples d'entiers $\left(\mu_{1}, \mu_{2}\right)$ vérifiant $0 \leqslant \mu_{2} \leqslant \mu_{1} \leqslant e$ et $\mu_{1}+\mu_{2} \equiv 0(\bmod b-1)$. Le calcul devient alors pénible et conduit à distinguer de nombreux cas; nous ne le faisons pas. Il est quand même possible à peu de frais d'obtenir le résultat suivant.

Proposition 4.2. On suppose $d=2$ et $h \geqslant 0$. Alors pour tout entier $e \geqslant 0$, on a :

$$
\operatorname{dim}_{k} \mathcal{X}_{\leqslant e+\left(b^{2}-1\right)}=\operatorname{dim}_{k} \mathcal{X}_{\leqslant e}+(b-1) .
$$


Remarque 4.3. La proposition signifie exactement que, dans le théorème 4.1, on peut choisir $N=b^{2}-1$ et que l'égalité énoncée vaut alors pour tout $e$.

Démonstration. On considère un couple $\left(\mu_{1}, \mu_{2}\right)$ pour lequel les variétés $\mathcal{X}_{\leqslant e}$ et $\mathcal{X}_{\left(\mu_{1}, \mu_{2}\right)}$ ont même dimension. Alors par la formule (4.2), on a $\operatorname{dim}_{k} \mathcal{X}_{\left(\mu_{1}+b^{2}-1, \mu_{2}\right)}=\operatorname{dim}_{k} \mathcal{X}_{\left(\mu_{1}, \mu_{2}\right)}+(b-1)$. Il en résulte l'inégalité $\operatorname{dim}_{k} \mathcal{X}_{\leqslant e+\left(b^{2}-1\right)} \geqslant \operatorname{dim}_{k} \mathcal{X}_{\leqslant e}+(b-1)$. On pose à présent $e^{\prime}=e+\left(b^{2}-1\right)$ et on choisit $\left(\mu_{1}^{\prime}, \mu_{2}^{\prime}\right)$ tel que $\operatorname{dim}_{k} \mathcal{X}_{\leqslant e^{\prime}}=\mathcal{X}_{\left(\mu_{1}^{\prime}, \mu_{2}^{\prime}\right)}$. Comme $e^{\prime} \geqslant b^{2}-1$, on a :

$$
b-1=\operatorname{dim}_{k} \mathcal{X}_{\leqslant b^{2}-1} \leqslant \operatorname{dim}_{k} \mathcal{X}_{\leqslant e^{\prime}} \leqslant \frac{\mu_{1}^{\prime}-\mu_{2}^{\prime}}{b+1}
$$

d'où $\mu_{1}^{\prime}-\left(b^{2}-1\right) \geqslant \mu_{2}^{\prime}$. La dimension de la variété $\mathcal{X}_{\left(\mu_{1}^{\prime}-\left(b^{2}-1\right), \mu_{2}^{\prime}\right)}$ peut donc encore se calculer par la formule 4.2) et elle vaut $\operatorname{dim}_{k} \mathcal{X}_{\left(\mu_{1}^{\prime}, \mu_{2}^{\prime}\right)}-(b-1)$. À partir de là, on déduit $\operatorname{dim}_{k} \mathcal{X}_{\leqslant e} \geqslant \operatorname{dim}_{k} \mathcal{X}_{\leqslant e^{\prime}}-(b-1)$, et la proposition est démontrée.

En dimension 3 On considère $\left(\mu_{1}, \mu_{2}, \mu_{3}\right)$ un triplet d'entiers tels que $\mu_{1} \geqslant \mu_{2} \geqslant \mu_{3}$ et $\mu_{1}+\mu_{2}+\mu_{3} \equiv 0$ $(\bmod b-1)$. De façon similaire à ce qui se passait en dimension 2 , calculer la dimension de $\mathcal{X}_{\left(\mu_{1}, \mu_{2}, \mu_{3}\right)}$ revient à maximiser la quantité $\operatorname{dim}(\mu)=2 \mu_{1}+\mu_{2}-\mu_{2,2}-\mu_{2,3}-\mu_{3,3}$ sous les contraintes

$$
\left\{\begin{array}{l}
\mu_{3} \leqslant \mu_{2,3} \leqslant \mu_{2} \leqslant \mu_{2,2} \leqslant \mu_{1} \quad ; \quad \mu_{2,3} \leqslant \mu_{3,3} \leqslant \mu_{2,2} \\
(b+1) \mu_{3,3} \geqslant b \mu_{2,2}+\mu_{2,3} \\
(b+1) \mu_{2,2}+2 \mu_{2,3}-\mu_{3,3} \geqslant b \mu_{1}+\mu_{2}+\mu_{3} \\
2 b \mu_{2,2}+(b+1) \mu_{2,3}-b \mu_{3,3} \geqslant b \mu_{1}+b \mu_{2}+\mu_{3} \\
\mu_{2,2}+\mu_{2,3} \equiv \mu_{3,3} \equiv 0 \quad(\bmod b-1)
\end{array}\right.
$$

À $\mu_{2,2}$ et $\mu_{2,3}$ fixés, le meilleur $\mu_{3,3}$ (i.e. le plus petit) est toujours $(b-1) \cdot\left\lceil\frac{b \mu_{2,2}+\mu_{2,3}}{b^{2}-1}\right\rceil$. On peut ainsi reformuler le problème en éliminant la variable $\mu_{3,3}$; celui-ci est équivalent à maximiser la somme

$$
2 \mu_{1}+\mu_{2}-\mu_{2,2}-\mu_{2,3}-(b-1) \cdot\left\lceil\frac{b \mu_{2,2}+\mu_{2,3}}{b^{2}-1}\right\rceil
$$

sous les nouvelles contraintes

$$
\left\{\begin{array}{l}
\mu_{3} \leqslant \mu_{2,3} \leqslant \mu_{2} \leqslant \mu_{2,2} \leqslant \mu_{1} \\
(b+1) \mu_{3,3} \geqslant b \mu_{2,2}+\mu_{2,3} \\
(b+1) \mu_{2,2}+2 \mu_{2,3}-(b-1) \cdot\left\lceil\frac{b \mu_{2,2}+\mu_{2,3}}{b^{2}-1}\right\rceil \geqslant b \mu_{1}+\mu_{2}+\mu_{3} \\
2 b \mu_{2,2}+(b+1) \mu_{2,3}-b(b-1) \cdot\left\lceil\frac{b \mu_{2,2}+\mu_{2,3}}{b^{2}-1}\right\rceil \geqslant b \mu_{1}+b \mu_{2}+\mu_{3} \\
\mu_{2,2}+\mu_{2,3} \equiv 0 \quad(\bmod b-1)
\end{array}\right.
$$

L'écriture se simplifie encore si l'on effectue le changement de variables $x=\frac{b \mu_{2,2}+\mu_{2,3}}{b-1}$ et $y=\frac{\mu_{2,2}+\mu_{2,3}}{b-1}-$ $\left\lceil\frac{x}{b+1}\right\rceil$. En effet, on a alors

$$
\operatorname{dim}(\mu)=2 \mu_{1}+\mu_{2}-2(b-1) \cdot\left\lceil\frac{x}{b+1}\right\rceil-(b-1) y
$$

tandis que les contraintes deviennent :

$$
\left\{\begin{array}{l}
x, y \in \mathbb{Z} \\
\mu_{3} \leqslant-x+b \cdot\left\lceil\frac{x}{b+1}\right\rceil+b y \leqslant \mu_{2} \leqslant x-\left\lceil\frac{x}{b+1}\right\rceil-y \leqslant \mu_{1} \\
x+y \geqslant \frac{b \mu_{1}+\mu_{2}+\mu_{3}}{b-1} \quad ; \quad x+b y \geqslant \frac{b \mu_{1}+b \mu_{2}+\mu_{3}}{b-1}
\end{array}\right.
$$

On laisse momentanément de côté les inégalités compliquées de la seconde ligne pour se concentrer sur celles de la troisième. Sur la figure $\bigoplus$ est représentée la région définie par celles-ci (en gris) et sont notées les 


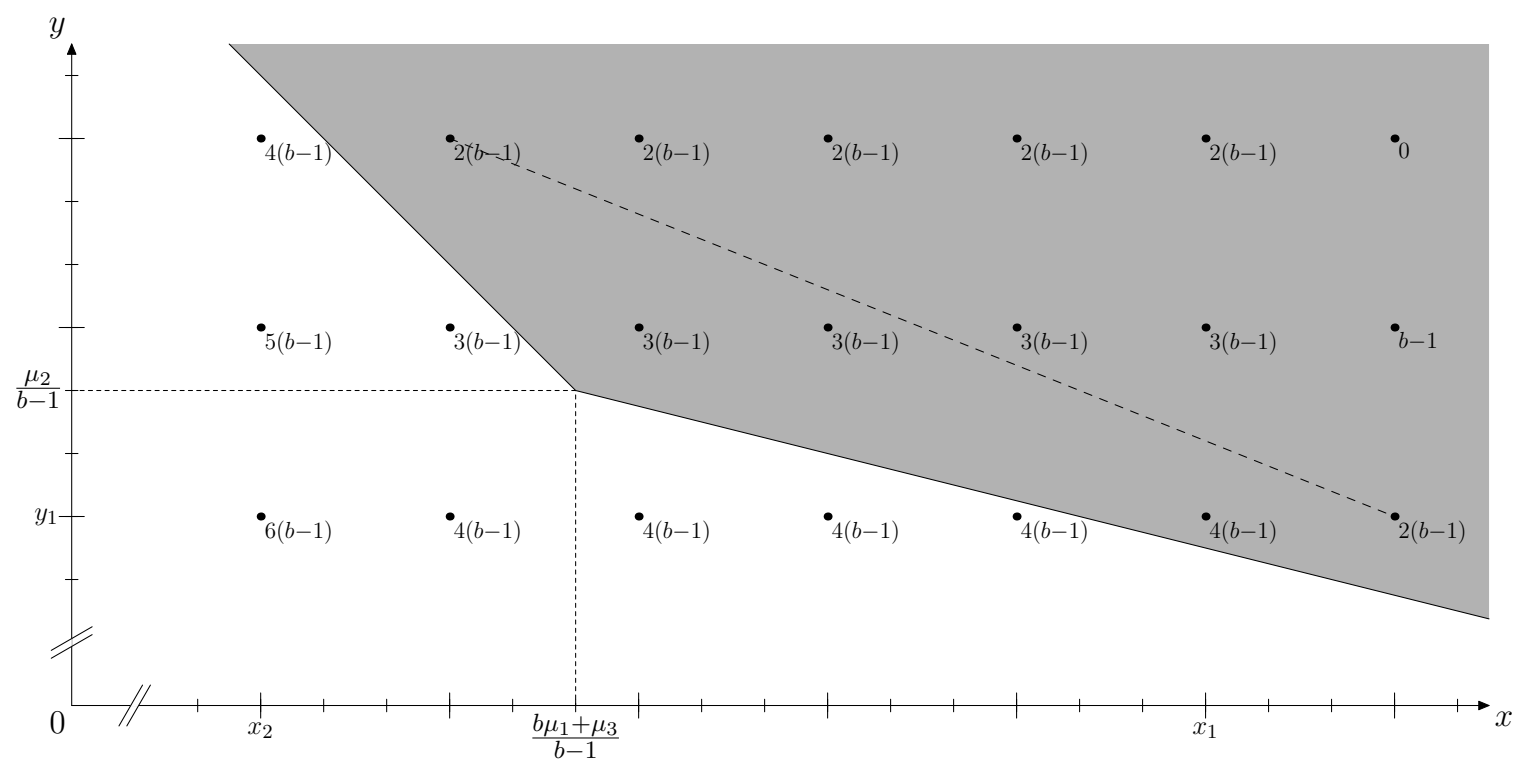

FIGURE 4 - Illustration du problème d'optimisation pour $b=4$

valeurs de la fonction à maximiser (à une constante additive près). En étudiant cette figure — et notamment en comparant les pentes des droites définissant le domaine à celle de la droite oblique en pointillés qui relie deux points de même valeur — on démontre que le maximum est nécessairement atteint au point de coordonnées $\left(x_{1}, y_{1}\right)$ avec

$$
x_{1}=(b+1) \cdot\left\lceil\frac{b \mu_{1}+\mu_{3}}{b^{2}-1}\right\rceil \quad \text { et } \quad y_{1}=\left\lceil\frac{\mu_{2}}{b-1}-\frac{b+1}{b} \cdot \operatorname{def}\left(\frac{b \mu_{1}+\mu_{3}}{b^{2}-1}\right)\right\rceil
$$

ou au point de coordonnées $\left(x_{2}, y_{2}\right)$ avec

$$
x_{2}=x_{1}-(b+1) \quad \text { et } \quad y_{2}=b+1+\left\lceil\frac{\mu_{2}}{b-1}-(b+1) \cdot \operatorname{def}\left(\frac{b \mu_{1}+\mu_{3}}{b^{2}-1}\right)\right\rceil \text {. }
$$

Plus précisément, le maximum est atteint en $\left(x_{1}, y_{1}\right)$ si $\operatorname{def}\left(\frac{b \mu_{1}+\mu_{3}}{b^{2}-1}\right) \leqslant \frac{b}{b+1}$ et en $\left(x_{2}, y_{2}\right)$ dans le cas contraire. Dans la suite, on notera $\left(x_{0}, y_{0}\right)$ ce point. Au sujet de la valeur du maximum, un calcul montre qu'il vaut :

$$
2 \mu_{1}+\mu_{2}-2(b-1) \cdot\left\lceil\frac{b \mu_{1}+\mu_{3}}{b^{2}-1}\right\rceil-(b-1) \cdot\left\lceil\frac{\mu_{2}}{b-1}-(b+1) m\right\rceil
$$

avec

$$
m=\min \left\{\frac{1}{b} \cdot \operatorname{def}\left(\frac{b \mu_{1}+\mu_{3}}{b^{2}-1}\right), \operatorname{def}\left(\frac{b \mu_{1}+\mu_{3}}{b^{2}-1}\right)-\frac{b-1}{b+1}\right\} .
$$

On rappelle quand même que certaines contraintes avaient été mises de côté. Il faut donc encore au moins se demander à quelles conditions les $x_{0}$ et $y_{0}$ précédents les satisfont. On remarque pour cela que $b+1$ divise $x_{0}$ de sorte que $\left\lceil\frac{x_{0}}{b+1}\right\rceil=\frac{x_{0}}{b+1}$ et que l'on a les encadrements suivants :

$$
\frac{b \mu_{1}+\mu_{3}}{b-1}-1 \leqslant x_{0} \leqslant \frac{b \mu_{1}+\mu_{3}}{b-1}+b \quad \text { et } \quad \frac{\mu_{2}}{b-1}-1 \leqslant y_{0} \leqslant \frac{\mu_{2}}{b-1}+1 .
$$

Ainsi il vient :

$$
\begin{aligned}
& \frac{-b \mu_{1}+b(b+1) \mu_{2}-\mu_{3}}{b^{2}-1}-\frac{b^{2}+2 b}{b+1} \leqslant-x_{0}+b\left\lceil\frac{x_{0}}{b+1}\right\rceil+b y_{0} \leqslant \frac{-b \mu_{1}+b(b+1) \mu_{2}-\mu_{3}}{b^{2}-1}+\frac{b^{2}+b+1}{b+1} \\
& \frac{b^{2} \mu_{1}-(b+1) \mu_{2}+b \mu_{3}}{b^{2}-1}-\frac{2 b+1}{b+1} \leqslant x_{0}-\left\lceil\frac{x_{0}}{b+1}\right\rceil-y_{0} \leqslant \frac{b^{2} \mu_{1}-(b+1) \mu_{2}+b \mu_{3}}{b^{2}-1}+\frac{b^{2}+b+1}{b+1}
\end{aligned}
$$


à partir de quoi il suit que le couple $\left(x_{0}, y_{0}\right)$ est solution du problème dès que le triplet $\left(\mu_{1}, \mu_{2}, \mu_{3}\right)$ vérifie $\mu_{1}-\mu_{2} \leqslant b\left(\mu_{2}-\mu_{3}\right)-\left(b^{2}+b+1\right)(b-1)$ et $\mu_{2}-\mu_{3} \leqslant b\left(\mu_{1}-\mu_{2}\right)-\left(b^{2}+b+1\right)(b-1)$, ce qui revient encore à dire que le triplet $\left(\mu_{1}-b^{2}-b-1, \mu_{2}, \mu_{3}+b^{2}+b+1\right)$ est $b$-régulier. On a ainsi démontré la proposition suivante.

Proposition 4.4. On suppose $h \geqslant 0$. Soit $\left(\mu_{1}, \mu_{2}, \mu_{3}\right)$ un triplet tel que $\left(\mu_{1}-b^{2}-b-1, \mu_{2}, \mu_{3}+b^{2}+\right.$ $b+1)$ soit intégralement b-régulier (voir définition 3 de l'introduction). Alors la dimension de la variété $\mathcal{X}_{\left(\mu_{1}, \mu_{2}, \mu_{3}\right)}$ est donnée par la formule (4.3).

À partir de la formule (4.3), on voit que, dans le cas où $\left(\mu_{1}-b^{2}-b-1, \mu_{2}, \mu_{3}+b^{2}+b+1\right)$ est intégralement $b$-régulier, la dimension de $\mathcal{X}_{\left(\mu_{1}, \mu_{2}, \mu_{3}\right)}$ est la somme du terme attendu $\frac{2\left(\mu_{1}-\mu_{3}\right)}{b+1}$ et d'une quantité bornée qui ne dépend que des congruences de $b \mu_{1}+\mu_{3}$ modulo $b^{2}-1$ et $\mu_{2}$ modulo $b-1$.

\subsection{Généralisations envisageables}

\subsection{1 À un opérateur $\sigma: M \rightarrow M$ arbitraire}

Dans tout cet article, on a supposé que $\sigma$ agissait coordonnée par coordonnée sur $M$. Ceci est en fait assez restrictif, et une situation plus générale que l'on aimerait étudier (notamment car elle correspond à certains problèmes importants de déformation) est celle où on se donne une application $\sigma$-semi-linéaire quelconque $\sigma_{M}: M \rightarrow M$. Dans ce cas, les variétés $\mathcal{X}_{\leqslant e}\left(\sigma_{M}\right), \mathcal{X}_{\mu}\left(\sigma_{M}\right)$ et $\mathcal{X}_{\leqslant \mu}\left(\sigma_{M}\right)$ sont définies de façon analogue. Par exemple, l'ensemble des $k$-points de $\mathcal{X}_{\leqslant e}\left(\sigma_{M}\right)$ est l'ensemble des réseaux $L$ de $M$ satisfaisant

$$
u^{e} L \subset \sigma_{M}^{\star}\left(k[[u]] \otimes_{\sigma, k[[u]]} L\right) \subset L
$$

où $\sigma_{M}^{\star}: k[[u]] \otimes_{\sigma, k[[u]]} M \rightarrow M$ est l'application linéarisée de $\sigma_{M}$. Si $A$ désigne la matrice de $\sigma_{M}$ dans une $k((u))$-base de $M$ (par exemple la base canonique), on s'autorisera à écrire $\mathcal{X}_{\leqslant e}(A)$ à la place de $\mathcal{X}_{\leqslant e}\left(\sigma_{M}\right)$, et de même pour les deux autres variantes. L'auteur pense que les théorèmes 1 , 4 et 5 s'étendent sans grande modification à ce cas plus général.

Conjecture 4.5. Il existe des constantes $b_{0}, c_{1}, \ldots, c_{7}$ et un vecteur $\mu_{0} \in \mathbb{R}^{d}$ tel que si $b \geqslant b_{0}$, alors

- pour tout entier e, on ait :

$$
\operatorname{dim}_{k} \mathcal{X}_{\leqslant e}\left(\sigma_{M}\right) \leqslant c_{1}+\left[\frac{d^{2}}{4}\right] \cdot \frac{e}{b+1}
$$

- pour tout entier e suffisamment grand, on ait :

$$
\operatorname{dim}_{k} \mathcal{X}_{\leqslant e}\left(\sigma_{M}\right) \geqslant-c_{2}+\left[\frac{d^{2}}{4}\right] \cdot \frac{e}{b+1}
$$

- pour tout $\mu=\left(\mu_{1}, \ldots, \mu_{d}\right) \in \mathbb{R}^{d}$ tel que $\mu_{1} \geqslant \cdots \geqslant \mu_{d}$ et $\mu_{1}+\cdots+\mu_{d} \equiv \operatorname{val}\left(\operatorname{det} \sigma_{M}\right)(\bmod b-$ 1) 8 on ait :

$$
\operatorname{dim}_{k} \mathcal{X}_{\mu}\left(\sigma_{M}\right) \leqslant c_{3}+(b-1) \cdot \sum_{i=1}^{d} \sum_{n=1}^{\infty} \mu_{i} \cdot \frac{d+1-i-w^{n}(i)}{b^{n}}
$$

- pour tout $\mu$ comme précedemment tel qu'en outre $\mu_{i} \geqslant \mu_{i+1}+c_{4}$ pour tout $i$, on ait si $d \geqslant 3$ :

$$
\begin{gathered}
\operatorname{dim}_{k} \mathcal{X}_{\mu}\left(\sigma_{M}\right) \geqslant-c_{5}+(b-1) \cdot \sum_{i=1}^{d} \sum_{n=1}^{\infty} \mu_{i} \cdot \frac{d+1-i-w^{n}(i)}{b^{n}} \\
- \text { pour tout } \mu=\left(\mu_{1}, \ldots, \mu_{d}\right) \in \mathbb{R}^{d} \text { tel que } \mu_{1} \geqslant \cdots \geqslant \mu_{d} \text {, on ait : } \\
-c_{6}+\sup _{\substack{\mu^{\prime} \leqslant \mu \\
\mu^{\prime}-\mu_{0} b \text {-rég. }}} \frac{\left\langle 2 \vec{\rho} \mid \mu^{\prime}\right\rangle_{d}}{b+1} \leqslant \operatorname{dim}_{k} \mathcal{X}_{\leqslant \mu}\left(\sigma_{M}\right) \leqslant c_{7}+\sup _{\substack{\mu^{\prime} \leqslant \mu \\
\mu^{\prime} b \text {-rég. }}} \frac{\left\langle 2 \vec{\rho} \mid \mu^{\prime}\right\rangle_{d}}{b+1} .
\end{gathered}
$$

8. Comme $\sigma_{M}$ est une application semi-linéaire, le déterminant de sa matrice peut varier lorsqu'on le calcule dans deux bases différentes ; toutefois la congruence modulo $b-1$ de sa valuation reste, elle, fixe. Il fait donc bien sens d'écrire que val(det $\left.\sigma_{M}\right)$ est congru à un certain entier modulo $b-1$. On notera également que dans le cas où $\mu_{1}+\cdots+\mu_{d}$ n'est pas congru à val(det $\left.\sigma_{M}\right)$ modulo $b-1$, la variété $\mathcal{X}_{\mu}$ est vide. 
La condition $d \geqslant 3$ peut paraître étrange, mais certains calculs explicites en dimension 2 (voir par exemple [5]) montrent que, dans ce cas, pour certains $\sigma_{M}$ (précisément, ceux qui conduisent à des objets simples) des congruences supplémentaires sur les $\mu_{i}$ doivent être imposées afin que la variété $\mathcal{X}_{\mu}\left(\sigma_{M}\right)$ résultante ne soit pas vide. Néanmoins, l'auteur pense - et certains calculs numériques tendent à le confirmer - qu'il s'agit là d'un phénomène lié à la petite dimension qui disparaît à partir de $d=3$.

\subsection{2 À d'autres propriétés géométriques}

Pour l'instant, seule la dimension des variétés $\mathcal{X}_{\leqslant e}, \mathcal{X}_{\mu}$ et $\mathcal{X}_{\leqslant \mu}$ a été regardée. Toutefois, d'autres propriétés géométriques revêtent également un intérêt certain. Il en est ainsi notamment du nombre de composantes connexes de ces variétés. À part pour le cas $d=2$ qui peut être traité à la main par des méthodes $a d$ hoc (voir [5] et [6]) et qui conduit déjà à des énoncés non triviaux, pratiquement rien n'est connu. De façon générale, étudier la géométrie fine des variétés précédentes paraît être une question très difficile. On peut néanmoins se demander dans quelle mesure les méthodes développées dans cet article sont susceptibles d'apporter une aide dans l'accomplissement de cette tâche. Si tout ce qui concerne l'optimisation linéaire semble lié exclusivement au calcul de la dimension, il est raisonnable de croire que la stratification par les variétés $\mathcal{X}_{\varphi}$ définie au $\$ 1$ ait encore un rôle à jouer pour d'autres questions, comme par exemple le calcul de la fonction zêta si le corps de base $k$ est fini ou de la caractéristique d'Euler-Poincaré.

En s'inspirant de la théorie de l'intégration motivique, on peut être encore plus précis. Soit $K_{0}\left(\operatorname{Var}_{k}\right)$ le groupe abélien présenté de la façon suivante :

- les générateurs sont les symboles $[X]$ où $X$ est un schéma de type fini sur $k$;

- les relations sont

$$
\begin{array}{ll}
{[X]=\left[X_{\text {red }}\right]} & \text { où } X_{\text {red }} \text { est le réduit de } X \\
{[X]=[Y]} & \text { si } X \text { et } Y \text { sont isomorphes } \\
{[X]=[U]+[F]} & \text { si } U \text { est un ouvert de } X \text { et } F \text { est le fermé complémentaire. }
\end{array}
$$

La formule $[X] \cdot[Y]=\left[X \times_{k} Y\right]$ définit un produit sur $K_{0}\left(\operatorname{Var}_{k}\right)$ qui est fait un anneau commutatif. L'élément neutre pour l'addition (resp. la multiplication) est le symbole de la variété vide (resp. du point). De même que l'on a considéré dans le $\S 4$.1 les séries génératrices des dimensions de $\mathcal{X}_{\leqslant e}, \mathcal{X}_{\mu}$ et $\mathcal{X}_{\leqslant \mu}$, on peut définir ici la série génératrice suivante :

$$
S\left(X_{1}, \ldots, X_{d}\right)=\sum_{\mu \in \mathbb{N}^{d}}\left[\mathcal{X}_{\mu}\left(\sigma_{M}\right)\right] \cdot X_{1}^{\mu_{1}} X_{2}^{\mu_{2}} \cdots X_{d}^{\mu_{d}}
$$

où $\sigma_{M}$ est un certain opérateur $\sigma$-semi-linéaire agissant sur $M$ et les $\mu_{i}$ sont les coordonnées de $\mu$. Bien sûr, on peut également considérer les séries génératrices associées aux variétés $\mathcal{X}_{\leqslant \mu}\left(\sigma_{M}\right)$ et $\mathcal{X}_{\leqslant e}\left(\sigma_{M}\right)$ mais celles-ci se déduisent de la précédente à l'aide de manipulations algébriques élémentaires, et c'est pourquoi on se contente de celle de $\mathcal{X}_{\mu}\left(\sigma_{M}\right)$.

Si l'on note $\mathbb{L}=\left[\mathbb{A}_{k}^{1}\right]$ le symbole de la droite affine, des résultats ou conjectures classiques en intégration motivique stipulent que les séries du type de $S$ sont en fait des fractions rationnelles lorsque leurs coefficients sont vus dans le localisé $K_{0}\left(\operatorname{Var}_{k}\right)\left[\mathbb{L}^{-1}\right]$ (ou parfois encore, un certain complété de cet anneau). L'auteur pense qu'il est raisonnable d'énoncer une conjecture similaire dans la situation de cet article.

Conjecture 4.6. Il existe deux polynômes $P, Q \in K_{0}\left(\operatorname{Var}_{k}\right)\left[X_{1}, \ldots, X_{d}\right]$ tels que $Q(0, \ldots, 0)$ soit inversible dans $K_{0}\left(\operatorname{Var}_{k}\right)\left[\mathbb{L}^{-1}\right]$ et l'égalité

$$
S\left(X_{1}, \ldots, X_{d}\right)=\frac{P\left(X_{1}, \ldots, X_{d}\right)}{Q\left(X_{1}, \ldots, X_{d}\right)}
$$

ait lieu dans l'anneau $K_{0}\left(\operatorname{Var}_{k}\right)\left[\mathbb{L}^{-1}\right]\left[\left[X_{1}, \ldots, X_{d}\right]\right]$.

L'intérêt d'un tel énoncé est qu'il peut être spécialisé à un certain nombres d'invariants géométriques ou arithmétiques plus classiques. Plus précisément dès que l'on dispose d'un morphisme $f$ de $K_{0}\left(\operatorname{Var}_{k}\right)$ dans un anneau $A$ qui envoie $\mathbb{L}$ sur un élément inversible, sa véracité implique la rationalité de la série :

$$
\sum_{\mu \in \mathbb{N}^{d}} f\left(\left[\mathcal{X}_{\mu}\left(\sigma_{M}\right)\right]\right) \cdot X_{1}^{\mu_{1}} X_{2}^{\mu_{2}} \cdots X_{d}^{\mu_{d}}
$$


Or, il existe un certain nombre de tels morphismes $f$ intéressants. Si $k$ est un corps fini, il y a par exemple celui qui a un symbole $[X]$ associe le cardinal de $X(k)$, ou plus généralement la fonction zêta de $X$. Pour un corps $k$ quelconque, on dispose également d'exemples construits par voie cohomologique comme les nombres de Betti ou le polynôme de Poincaré virtuel. À partir de là, on peut retrouver la dimension de $X$ ou encore son nombre de composantes irréductibles de dimension maximale. La conjecture 4.6 admet donc pour conséquence la rationalité de la série génératrice des dimensions (qui a été démontrée directement dans cet article), mais implique également la rationalité d'autres séries génératrices numériques.

Finalement, pour étudier d'autres propriétés géométriques qui ne proviennent pas de $K_{0}\left(\operatorname{Var}_{k}\right)$, il pourrait être intéressant de comprendre comment les variétés $\mathcal{X}_{\varphi}$ s'agencent entre elles à l'intérieur de $\mathcal{X}_{\leqslant e}, \mathcal{X}_{\mu}$ ou $\mathcal{X}_{\leqslant \mu}$. Notamment, une question qui paraît importante est de déterminer l'adhérence de $\mathcal{X}_{\varphi}$ à l'intérieur de ces variétés. Par exemple, s'écrit-elle comme une union de certains $\mathcal{X}_{\varphi^{\prime}}$ où $\varphi^{\prime}$ vérifie une condition qui s'exprime facilement en fonction de $\varphi$ ?

\subsection{3 À un groupe réductif connexe arbitraire}

À l'instar des variétés de Deligne-Lusztig, il est possible d'étendre la définition des variétés $\mathcal{X}_{\mu}$ et $\mathcal{X}_{\leqslant \mu}$ à un groupe réductif connexe déployé quelconque (le cas qui a été considéré dans cet article étant celui de $\mathrm{GL}_{d}$ ). Plus précisément, on considère un groupe réductif connexe $G$ défini sur le corps $k$ (qui, pour simplifier, est encore supposé algébriquement clos) et $T \subset G$ un tore maximal. Soit $X_{\star}(T)$ le groupe des caractères de $T$. On fixe une chambre de Weyl dans $X_{\star}(T) \otimes \mathbb{R}$ dont l'adhérence est notée $C$. Si $\lambda \in X_{\star}(T)$, on appelle $u^{\lambda}$ l'image de $u \in \mathbb{G}_{m}(K)$ dans $T(K) \subset G(K)$, où $K=k((u))$. Si on pose $\mathcal{O}_{K}=k[[u]]$, la décomposition de Cartan dit que $G(K)$ s'écrit comme l'union disjointe des doubles classes $G\left(\mathcal{O}_{K}\right) u^{\mu} G\left(\mathcal{O}_{K}\right)$ où $\mu$ parcourt l'ensemble des copoids dominants. On définit par ailleurs un opérateur $\sigma$ agissant sur $G(K)$ de la façon suivante : on fixe un morphisme de groupes algébriques $\sigma_{0}: G \rightarrow G$ qui induit une bijection sur les $k$-points (on rappelle que $k$ est supposé algébriquement clos) et on pose $\sigma=$ $G\left(u \mapsto u^{b}\right) \circ \sigma_{0}(K)$ où $\sigma_{0}(K)$ désigne l'application induite par $\sigma_{0}$ sur les $K$-points et où $G\left(u \mapsto u^{b}\right)$ est l'application déduite par fonctorialité du morphisme d'anneaux $K \rightarrow K, \sum_{i \gg-\infty} a_{i} u^{i} \mapsto \sum_{i \gg-\infty} a_{i} u^{b i}$. Si $\mu$ est un copoids dominant et si $A \in G(K)$, on peut alors définir des variétés $\mathcal{X}_{\mu}^{G}(A)$ dont les $k$-points sont :

$$
\mathcal{X}_{\mu}^{G}(A)(k)=\left\{g \in G(K) / G\left(\mathcal{O}_{K}\right) \mid g^{-1} A \sigma(g) \in G\left(\mathcal{O}_{K}\right) u^{\mu} G\left(\mathcal{O}_{K}\right)\right\} .
$$

On définit également $\mathcal{X}_{\leqslant \mu}^{G}(A)$ comme la réunion des $\mathcal{X}_{\mu^{\prime}}^{G}(A)$ où $\mu^{\prime}$ décrit l'ensemble des copoids dominants tels que $\mu-\mu^{\prime}$ s'écrive comme une combinaison linéaire à coefficients positifs des racines simples correspondant au choix de $C$. Si $G$ est le groupe linéaire $\mathrm{GL}_{d}$, on retrouve les variétés $\mathcal{X}_{\mu}(A)$ et $\mathcal{X}_{\leqslant \mu}(A)$. De façon générale, les variétés $\mathcal{X}_{\mu}^{G}(A)$ et $\mathcal{X}_{\leqslant \mu}^{G}(A)$ sont toujours de dimension finie, et on peut s'interroger sur la valeur de cette dimension.

Dans cette optique, une première question est de savoir si le théorème $€$ a des chances de se généraliser à cette nouvelle situation, et le cas échéant sous quelle forme. Un premier coup d'œil à l'expression

$$
(b-1) \cdot \min _{w \in \mathfrak{S}_{d}}\left\langle\vec{\rho}_{w} \mid \mu\right\rangle_{d} \quad \text { où } \quad \vec{\rho}_{w}=\left(\sum_{n=1}^{\infty} \frac{d+1-i-w^{n}(i)}{b^{n}}\right)_{1 \leqslant i \leqslant d} \in \mathbb{R}^{d}
$$

qui apparaît dans son énoncé (et qui constitue une première approximation de la dimension de $\mathcal{X}_{\mu}$ ) laisse bon espoir. En effet, on voit d'emblée apparaître un minimum pris sur le groupe des permutations de $\{1, \ldots, d\}$, c'est-à-dire exactement sur le groupe de Weyl de $\mathrm{GL}_{d}$. En outre si l'on fait agir ce groupe de manière naturelle sur $\mathbb{R}^{d}$ — c'est-à-dire par $w \cdot\left(y_{1}, \ldots, y_{d}\right)=\left(y_{w^{-1}(1)}, \ldots, y_{w^{-1}(d)}\right)$ - le vecteur $\vec{\rho}_{w}$ s'exprime en fonction de $\vec{\rho}=\left(\frac{d+1}{2}-i\right)_{1 \leqslant i \leqslant d}$ comme suit :

$$
\vec{\rho}_{w}=(b-1) \cdot \sum_{n=1}^{\infty} \frac{\vec{\rho}+w^{-n} \vec{\rho}}{b^{n}}=\vec{\rho}+(b-1) \cdot(b w-1)^{-1}(\vec{\rho})
$$

au moins lorsque $b$ est assez grand pour que l'endomorphisme $b w-1 \mathrm{de} \mathbb{R}^{d}$ soit inversible. Si l'on se rappelle finalement que $\vec{\rho}$ est égal à la demi-somme des racines positives du système de racines $A_{d}$, on voit que la formule (4.4) s'exprime uniquement en termes du système de racines du groupe $\mathrm{GL}_{d}$. Ces considérations conduisent à la conjecture suivante. 
Conjecture 4.7. Soit $G$ un groupe réductif connexe sur $k$. Soit $T$ un tore maximal de $G$. On note $W$ le groupe de Weyl associé et on fixe une fois pour toutes le choix d'une chambre de Weyl. Soient $\vec{\rho}$ la demisomme des racines positives de $G$ et $A \in G(K)$. Alors, il existe des constantes $b_{0}$ et $c_{0}$ telles que pour tout $b \geqslant b_{0}$, on ait :

$$
\operatorname{dim}_{k} \mathcal{X}_{\mu}^{G}(A) \leqslant c_{0}+\inf _{w \in W}\left\langle\vec{\rho}_{w} \mid \mu\right\rangle \quad \text { où } \quad \vec{\rho}_{w}=\vec{\rho}+(b-1) \cdot(b w-1)^{-1}(\vec{\rho})
$$

pour tout copoids dominant $\mu$.

Remarque 4.8. Lorsque $w$ est le mot le plus long $w_{0}$ de $W$, le vecteur $\rho_{w_{0}}$ se calcule facilement. En effet, $w_{0}$ échange les racines positives avec les racines négatives. En particulier, on a $w_{0}(\vec{\rho})=-\vec{\rho}$, d'où il résulte que $\left(b w_{0}-1\right)(\vec{\rho})=-(b+1) \vec{\rho}$, et par suite que $\vec{\rho}_{w_{0}}=\frac{2 \vec{\rho}}{b+1}$; on retrouve donc encore une fois ce vecteur particulier.

Il paraît aussi raisonnable de croire qu'une minoration de la dimension de $\mathcal{X}_{\mu}^{G}(A)$ par une expression du même type soit valable, au moins lorsque $\mu$ vérifie une certaine condition d'intégrité et reste suffisamment lors de la frontière de $C$. Malgré tout, gardant à l'esprit le comportement singulier des variétés $\mathcal{X}_{\mu}\left(\sigma_{M}\right)$ lorsque $\sigma_{M}$ représente un $\sigma$-module simple en dimension 2, nous préférons rester prudent et évasif à ce sujet.

La conjecture donne également une indication sur la façon d'étendre la définition de copoids $b$-réguliers : un copoids dominant $\mu$ est dit b-régulier lorsque le minimum des produits scalaires $\left\langle\vec{\rho}_{w} \mid \mu\right\rangle(w \in W)$ est atteint lorsque $w$ est le mot le plus long $w_{0}$, ce qui s'écrit en déroulant les définitions :

$$
\left\langle\vec{\rho}+(b+1) \cdot(b w-1)^{-1}(\vec{\rho}) \mid \mu\right\rangle \geqslant 0
$$

pour tout $w \in W$. Cette définition s'étend à tous les $\mu \in X_{\star}(T) \otimes \mathbb{R}$. On peut démontrer que, si $b$ est assez grand, un copoids $\mu$ est $b$-régulier si, et seulement s'il vérifie les inégalités (4.5) pour tout $w \in W$ de longueur $\ell\left(w_{0}\right)-1$. Par ailleurs, pour $b$ suffisamment grand, la suite des copoids $b$-réguliers est croissante en $b$ (i.e. si $\mu$ est $b$-régulier, alors il est $b^{\prime}$-régulier pour tout $b^{\prime} \geqslant b$ ) et tout copoids $b$-régulier est dominant, dans le sens où il appartient à $C$. Réciproquement, si $\mu$ est un élément de l'intérieur de $C$ (c'est-à-dire un élément de la chambre de Weyl choisie), il est $b$-régulier pour $b$ suffisamment grand (le $\ll$ suffisamment $\gg$ dépendant bien sûr de $\mu$ ).

On a également une conjecture pour les variétés $\mathcal{X}_{\leqslant \mu}^{G}(A)$ :

Conjecture 4.9. Soit $G$ un groupe réductif connexe sur $k$. Soit $T$ un tore maximal de $G$. On note $W$ le groupe de Weyl associé et on fixe une fois pour toutes le choix d'une chambre de Weyl. Soient $\vec{\rho}$ la demisomme des racines positives de $G$ et $A \in G(K)$. Alors, il existe des constantes $c_{1}$ et $c_{2}$ et un élément $\mu_{0} \in X_{\star}(T) \otimes \mathbb{R}$ tels que :

$$
-c_{2}+\sup _{\substack{\mu^{\prime} \leqslant \mu \\ \mu^{\prime}-\mu_{0} b \text {-rég. }}} \frac{\left\langle 2 \vec{\rho} \mid \mu^{\prime}\right\rangle_{d}}{b+1} \leqslant \operatorname{dim}_{k} \mathcal{X}_{\leqslant \mu}^{G}(A) \leqslant c_{1}+\sup _{\substack{\mu^{\prime} \leqslant \mu \\ \mu^{\prime} \text {-rég. }}} \frac{\left\langle 2 \vec{\rho} \mid \mu^{\prime}\right\rangle_{d}}{b+1}
$$

où $\mu^{\prime}$ désigne, ici, un copoids réel.

Un cas particulièrement intéressant, qui apparaît déjà dans l'article de Kisin [7], est celui où l'on suppose le corps $k$ parfait (par exemple $k=\mathbb{F}_{p}$ ), où l'on se donne une extension finie $\ell$ de $k$ et où l'on considère le groupe $G$ défini comme la restriction des scalaires à la Weil de $\ell$ à $k$ de $G_{d}$. Les variétés obtenues ont alors encore une interprétation arithmétique puisqu'elles apparaissent comme certaines espaces de modules de schémas en groupes définis sur des corps locaux. Lorsque $d=2$, le calcul de leur dimension a déjà été accompli par Imai dans [11] et, dans ce cas, les résultats qu'il obtient sont en accord avec les conjectures précédentes.

\section{Références}

[1] C. Breuil, Schémas en groupes et corps des normes, disponible à http://www.ihes.fr/ breuil/publications.html, 1998 
[2] X. Caruso, Sur la classification de quelques $\sigma$-modules simples, Mosc. Math. J. 9 (2009)

[3] E. Gawrilow, M. Joswig, polymake : a Framework for Analyzing Convex Polytopes in Polytopes Combinatorics and Computation, Birkhhäuser (2000), pp. 43-74

[4] U. Görtz, T. Haines, R. Kottwitz, D. Reuman, Dimensions of some affine Deligne-Lusztig varieties, Ann. Scient. Éc. Norm. Sup. 39 (2006), pp. 467-511

[5] E. Hellmann, On the structure of some moduli spaces of finite flat group schemes, Mosc. Math. J. 9 (2009), pp. 531-561

[6] E. Hellmann, Connectedness of Kisin varieties for $G L_{2}$, preprint (2010)

[7] M. Kisin, Moduli of finite flat group schemes and modularity, Annals of Math. 170 (2009), pp. 10851180.

[8] J. Le Borgne, Un algorithme pour la réduction des $\sigma$-modules sur $k((u))$, en préparation

[9] N. Imai, On the connected components of moduli spaces of finite flat models, à paraître à Amer. J. Math.

[10] N. Imai, Finite flat models of constant group schemes of rank two, preprint (2008)

[11] N. Imai, Ramification and moduli spaces of finite flat models, preprint (2008)

[12] D. Monniaux. A quantifier elimination algorithm for linear real arithmetic, in LPAR (Logic for Programming Artificial Intelligence and Reasoning), Lecture Notes in Computer Science 5330, pp. 243257.

[13] C. H. Papadimitriou, K. Steiglitz The Max-Flow, Min-Cut Theorem in Combinatorial Optimization : Algorithms and Complexity, Dover (1998), pp. 120-128.

[14] A. Rényi, Théorie des éléments saillants d'une suite d'observations, Ann. Fac. Sci. Univ. ClermontFerrand 8 (1962), pp. 7-13

[15] G. Pappas, M. Rapoport, Ф-modules and coefficient spaces, Mosc. Math. J. 9 (2009), pp. 625-663

[16] E. Viehmann, The dimension of some affine Deligne-Lusztig varieties, Ann. Scient. Éc. Norm. Sup. 39 (2006), pp. 513-526 